\title{
Revised value of the eighth-order QED contribution to the anomalous magnetic moment of the electron
}

\author{
T. Aoyama \\ Institute of Particle and Nuclear Studies, \\ High Energy Accelerator Research Organization (KEK), Tsukuba, Ibaraki 305-0801, Japan \\ M. Hayakawa \\ Department of Physics, Nagoya University, Nagoya, Aichi 464-8602, Japan \\ T. Kinoshita \\ Newman Laboratory for Elementary-Particle Physics, \\ Cornell University, Ithaca, New York 14853, U.S.A. \\ M. Nio \\ Theoretical Physics Laboratory, Nishina Center, \\ RIKEN, Wako, Saitama 351-0198, Japan
}

\begin{abstract}
We have carried out a new evaluation of the eighth-order contribution to the electron $g-2$ using FORTRAN codes generated by an automatic code generator GENCODEN. Comparison of the "new" result with the "old" one has revealed an inconsistency in the treatment of the infrared divergences in the latter. With this error corrected we now have two independent determinations of the eighth-order term. This leads to the revised value $1159652182.79(7.71) \times 10^{-12}$ of the electron $g-2$, where the uncertainty comes mostly from that of the best non-QED value of the fine structure constant $\alpha$. The new value of $\alpha$ derived from the revised theory and the latest experiment is $\alpha^{-1}=137.035999084$ (51) [0.37 ppb], which is about $4.7 \mathrm{ppb}$ smaller than the previous $\alpha^{-1}$.

PACS numbers: 13.40.Em,14.60.Cd,12.20.Ds,06.20.Jr
\end{abstract}




\section{INTRODUCTION AND SUMMARY}

The anomalous magnetic moment of the electron has played a central role in testing the validity of QED [1, 2]. The test became very stringent when the precision of measurement of the electron and positron was improved by three orders of magnitude over the best earlier result [3] by the University of Washington group in the Penning trap experiment [4]

$$
\begin{aligned}
& a_{e^{-}}=1159652188.4(4.3) \times 10^{-12} \quad[3.7 \mathrm{ppb}] \\
& a_{e^{+}}=1159652187.9(4.3) \times 10^{-12} \quad[3.7 \mathrm{ppb}],
\end{aligned}
$$

where $a_{e} \equiv(g-2) / 2$ and $g$ is the $g$-factor of electron. The main source of the remaining uncertainty in Eq. (1) is the uncontrolled shift of the frequency due to the resonance between the electron and the metal cavity of hyperbolic shape. Brown et al. [5] showed that this source of uncertainty can be reduced significantly using a metal trap with the cylindrical cavity whose resonance structure can be calculated analytically.

The recent Harvard measurement is based on the cylindrical cavity. Their value announced in 2006 is [ [6]

$$
a_{e}(\mathrm{HV} 06)=1159652180.85(0.76) \times 10^{-12} \quad[0.66 \mathrm{ppb}]
$$

which has a 5.5 times smaller uncertainty than the previous measurements listed in Eq. (11). Very recently, the same Harvard group has succeeded in reducing the uncertainty further by a factor 2.7 [7]:

$$
a_{e}(\mathrm{HV} 08)=1159652180.73(0.28) \times 10^{-12} \quad[0.24 \mathrm{ppb}]
$$

To match the precision of the measurement the theory of $a_{e}$ must include radiative corrections of up to the eighth-order of QED perturbation theory as well as the hadronic and weak contributions

$$
a_{e}=a_{e}(\mathrm{QED})+a_{e}(\text { hadron })+a_{e}(\text { weak }) .
$$

The hadronic [8, 9, 10, 11] and weak contributions [12] to $a_{e}$ are very small, but not entirely negligible relative to the measurement uncertainties (2) or (3):

$$
\begin{aligned}
& a_{e}(\text { hadron })=1.682(20) \times 10^{-12}, \\
& a_{e}(\text { weak })=0.0297(5) \times 10^{-12} .
\end{aligned}
$$


The QED contribution $a_{e}(\mathrm{QED})$ can be divided further into four parts taking account of the presence of other leptons:

$$
a_{e}(\mathrm{QED})=A_{1}+A_{2}\left(m_{e} / m_{\mu}\right)+A_{2}\left(m_{e} / m_{\tau}\right)+A_{3}\left(m_{e} / m_{\mu}, m_{e} / m_{\tau}\right),
$$

where $m_{e}, m_{\mu}$, and $m_{\tau}$ are masses of the electron $(e)$, muon $(\mu)$ and tau-lepton $(\tau)$, respectively. $A_{1}$, being dimensionless, depends only on the fine structure constant $\alpha . A_{2}$ denotes contributions from the Feynman diagrams which have closed loops of either muon or taulepton. $A_{3}$ stands for the contributions of the Feynman diagrams which contain both $\mu$ loop and $\tau$ loop. Each $A_{i}$ can be calculated by the QED perturbation theory

$$
A_{i}=A_{i}^{(2)}\left(\frac{\alpha}{\pi}\right)+A_{i}^{(4)}\left(\frac{\alpha}{\pi}\right)^{2}+A_{i}^{(6)}\left(\frac{\alpha}{\pi}\right)^{3}+\cdots .
$$

The purpose of this paper is to give a detailed account of derivation of the revised value of the eighth-order coefficient of $A_{1}$ reported recently [13]

$$
A_{1}^{(8)}=-1.9144(35) \text {. }
$$

Making use of our automating algorithms in handling ultraviolet (UV) and infrared (IR) divergences [14, 15], we are now able to generate the eighth-order FORTRAN codes very easily and swiftly. However, numerical evaluation of these codes is still nontrivial and requires a huge computational resource. Thus far the "new" calculation has achieved a relative uncertainty of about $3 \%$. Although this is still more than an order of magnitude less accurate than that of Ref. [16], it is good enough for the purpose of checking the old calculation.

Comparison of the "new" numerical result with the old one has revealed an inconsistency in the treatment of the IR divergence in the latter. With this error of the old calculation corrected, we now have two independent determinations of $A_{1}^{(8)}$. Of course, precise evaluation of all terms of "new" $A_{1}^{(8)}$ by the integration routine VEGAS [17] requires an enormous amount of computation. Fortunately, as is described in Sec. IVD, the correction term itself can be evaluated easily and very precisely. This is why we are able to give the uncertainty in Eq. (9) which is essentially identical with that of the previous calculation [16].

Besides $A_{1}^{(8)}$ the known terms of Eq. (7) are as follows [2, 18, 19, 20, 21, 22, 23, 24, 25, 
26, 27, 28, 29]:

$$
\begin{aligned}
& A_{1}^{(2)}=0.5, \\
& A_{1}^{(4)}=-0.328478965579 \cdots, \\
& A_{1}^{(6)}=1.181241456587 \cdots, \\
& A_{1}^{(10)}=0.0(4.6), \\
& A_{2}^{(4)}\left(m_{e} / m_{\mu}\right)=5.19738670(27) \times 10^{-7}, \\
& A_{2}^{(4)}\left(m_{e} / m_{\tau}\right)=1.83763(60) \times 10^{-9}, \\
& A_{2}^{(6)}\left(m_{e} / m_{\mu}\right)=-7.37394158(28) \times 10^{-6}, \\
& A_{2}^{(6)}\left(m_{e} / m_{\tau}\right)=-6.5819(19) \times 10^{-8}, \\
& A_{3}^{(6)}\left(m_{e} / m_{\mu}, m_{e} / m_{\tau}\right)=0.190945(62) \times 10^{-12} .
\end{aligned}
$$

Here, $A_{1}^{(2)}, A_{1}^{(4)}$, and $A_{1}^{(6)}$ are known analytically. $A_{2}^{(4)}, A_{2}^{(6)}$ and $A_{3}^{(6)}$ are known analytically as functions of mass ratios so that their uncertainties are due to those of measured lepton masses only. Note that $A_{1}^{(10)}$ is actually unknown and the value listed above is an educated guess calculated by the recipe proposed in Ref. [29] to indicate a likely range of the value taken by $A_{1}^{(10)}$. This will soon be replaced by a real number, which is being evaluated by FORTRAN codes generated with the help of the automatic code generator GENCODE $N$ [14, 15]. Until then $A_{1}^{(10)}$ in Eq. (10) is the largest source of theoretical uncertainty.

In order to obtain the numerical value of the theoretical $g-2$, an explicit value of the fine structure constant $\alpha$, which is determined by the physical phenomena other than $g-2$, is required. At present the best values of $\alpha$ available in the literature are from the Cesium atom experiments [30, 31] and the Rubidium atom experiment [32]

$$
\begin{aligned}
& \alpha^{-1}(\mathrm{Cs} 06)=137.03600000(110) \quad \text { [8.0ppb] } \\
& \alpha^{-1}(\mathrm{Rb} 06)=137.03599884(91) \quad[6.7 \mathrm{ppb}] .
\end{aligned}
$$

They lead to the theoretical predictions of $a_{e}$ :

$$
\begin{gathered}
a_{e}(\mathrm{Cs})=1159652172.99(0.10)(0.31)(9.32) \times 10^{-12}, \\
a_{e}(\mathrm{Rb})=1159652182.79(0.10)(0.31)(7.71) \times 10^{-12},
\end{gathered}
$$

respectively, where the uncertainty 0.10 comes from the eighth-order result (99), 0.31 is an estimated uncertainty of the tenth-order term, and 9.32 and 7.71 come from the uncertainties of the input values of the fine structure constants given in Eqs. (11) and (12). The 
uncertainty due to the hadronic and weak contributions (66) is $0.02 \times 10^{-12}$. The revised theoretical anomaly $a_{e}$ is in closer agreement with the experimental values (2) and (3) than the old value [16].

Unfortunately, the precision of $a_{e}$ given in Eq. (13) is not high enough for direct confrontation between the experimental and theoretical $a_{e}$ 's. This is because the uncertainties in $a_{e}$ due to these $\alpha$ 's amount to $9.3 \times 10^{-12}$ for $\alpha(\mathrm{Cs} 06)$ and $7.7 \times 10^{-12}$ for $\alpha(\mathrm{Rb06})$, respectively, which are an order of magnitude larger than the experimental uncertainty $0.76 \times 10^{-12}$ and the theoretical uncertainty $0.28 \times 10^{-12}$ of $a_{e}$.

This implies that, assuming the validity of QED, the electron $g-2$ is in fact the best source of the fine structure constant $\alpha$, an order of magnitude better than any alternative. Because of high precision of the experiments (2) and (3) the fine structure constant $\alpha$ determined from $a_{e}$ is rather sensitive to the revision of the theoretical prediction. Equating the Harvard measurements (2) or (3), and the theory (44), we obtain [7, 33, 34]

$$
\begin{aligned}
& \alpha^{-1}\left(a_{e}(\mathrm{HV} 06=\mathrm{Th} 07)\right)=137.035999070(12)(37)(90) \quad[0.71 \mathrm{ppb}], \\
& \alpha^{-1}\left(a_{e}(\mathrm{HV} 08=\mathrm{Th} 07)\right)=137.035999084(12)(37)(33) \quad[0.37 \mathrm{ppb}],
\end{aligned}
$$

where the first and second uncertainties come from the numerical uncertainties of $A_{1}^{(8)}$ and $A_{1}^{(10)}$, respectively, and the third in Eq. (14) or Eq. (15) comes from the experiment (2) or (3), respectively.

These values of $\alpha^{-1}$ are smaller than the old $\alpha^{-1}\left(a_{e}(\mathrm{HV} 06=\right.$ Th06) $)$ by $-6.41180(73) \times$ $10^{-7}$ which is about $4.7 \mathrm{ppb}$ (or about $7 \mathrm{s.} \mathrm{d}$.), but are still in good agreement with $\alpha^{-1}(\mathrm{Rb} 06)$ of Eq. (11) and $\alpha^{-1}(\mathrm{Cs} 06)$ of Eq. (12), whose uncertainties are about 7 ppb.

The organization of the paper is as follows. In Sec. II, we briefly overview the "old" and "new" approaches to the numerical calculation of the electron $g-2$ in QED. In Sec. III, the diagrams of Group $\mathrm{V}$ of the eighth-order term are discussed. We compared the results of the "old" and "new" calculations and found an unaccountable difference in the results of the diagram $M_{18}$. In Sec. IV, the diagram $M_{16}$ is closely examined instead of $M_{18}$. This is because $M_{16}$ has a similar structure to $M_{18}$, but somewhat simpler. We found a source of the discrepancy between the "old" and "new" results and the errors in the "old" calculation of $M_{16}$ and $M_{18}$ are corrected. Sec. V gives the summary of the updated value of the eighth-order contribution to the electron $g-2$.

Appendix A presents the tests of the automation system GENCODE $N$ for the fourth-order 
and sixth-order $g-2$ 's. Appendix B gives our renormalization scheme of the magnetic moment amplitude in the "new" approach. Similarly, Appendix C gives the renormalization scheme of the renormalization constants in the "new" approach.

\section{OLD VS NEW APPROACH}

The purpose of this paper is the presentation of the results of evaluation of $A_{1}^{(8)}$ by two independent methods. Although these methods started from the same Feynman-parametric representation of $A_{1}^{(8)}$, they took different approaches, in particular, in the handling of the self-energy subdiagrams and associated infrared (IR) divergences. Furthermore, the "new" approach was instrumental in discovering an error in the handling of infrared divergence in the old method [16]. After correcting this error, we now have two independent evaluations of $A_{1}^{(8)}$, enhancing substantially the credibility of the calculation.

\section{A. Common starting point}

The anomalous magnetic moment $a_{e}$ is given by the static limit of the magnetic form factor that is related to the proper vertex part $\Gamma^{\nu}$. Throughout this paper our attention is focused on the $q$-type diagrams, namely, proper vertex diagrams that have no closed lepton loops. In both old and "new" formulations, we use a relation derived from the WardTakahashi identity [35, 36]

$$
\Lambda^{\nu}(p, q) \simeq-q^{\mu}\left[\frac{\partial \Lambda_{\mu}(p, q)}{\partial q_{\nu}}\right]_{q \rightarrow 0}-\frac{\partial \Sigma(p)}{\partial p_{\nu}}
$$

between the self-energy part $\Sigma(p)$ and the sum of vertex parts $\Lambda^{\nu}(p, q)$ obtained by inserting an external vertex in the lepton lines of $\Sigma$ in all possible ways. Here, the momentum of the incoming lepton is $p-\frac{1}{2} q$ and that of the outgoing lepton is $p+\frac{1}{2} q$. By means of Eq. (16) a set of vertex diagrams are amalgamated into a single self-energy-like diagram, which reduces the number of independent integrals substantially. For the eighth-order $q$ type diagrams, the number of Feynman diagrams is reduced from 518 to 74 . Taking into account the time-reversal symmetry, the number is further reduced from 74 to 47.

The amplitude of the magnetic moment contribution of a diagram is obtained by applying Feynman-Dyson rules of QED in the momentum space. Carrying out the momentum 
integration analytically, we can express the amplitude of $2 n$ th-order diagram $\mathcal{G}$ as an integral over the Feynman parameters $z_{i}$ :

$$
\begin{aligned}
M_{\mathcal{G}}^{(2 n)}=\left(-\frac{1}{4}\right)^{n}(n-1) ! \int(d z)_{\mathcal{G}}\left[\frac{1}{n-1}\left(\frac{E_{0}+C_{0}}{U^{2} V^{n-1}}+\frac{E_{1}+C_{1}}{U^{3} V^{n-2}}+\cdots\right)\right. \\
\left.+\left(\frac{N_{0}+Z_{0}}{U^{2} V^{n}}+\frac{N_{1}+Z_{1}}{U^{3} V^{n-1}}+\cdots\right)\right],
\end{aligned}
$$

where $(d z)_{\mathcal{G}}=\prod_{i} d z_{i} \delta\left(1-\sum_{i} z_{i}\right)$. The factor $(\alpha / \pi)^{n}$ is omitted for simplicity.

The quantities $E_{k}, C_{k}, N_{k}$, and $Z_{k}$ are polynomials of symbols called building blocks $B_{i j}, A_{i}$, and $C_{i j}$ [35]. The symbols $B_{i j}$ and $U$ are homogeneous polynomials of Feynman parameters, related to the flow of loop momenta in the diagram. The symbol $A_{i}$ is called scalar current that is associated with the flow of external momenta. They are functions of $B_{i j}, U$, and $z_{i}$. The symbol $C_{i j}$ is given by $z_{i}, B_{i j}$ and $U$. The denominator function $V$ is defined by

$$
V=\sum_{i} z_{i}-G, \quad G=\sum_{i} z_{i} A_{i}
$$

where the summation is over the electron lines only, and the electron mass is chosen to unity for simplicity.

\section{B. Different structure of integrand}

Although the "old" and "new" methods have the common starting point, they have an important difference in practice. In the "old" version of the programs, the size of the integrand was reduced by taking symmetries of a diagram into account. One type of modifications was applied to the integrand by using 8 "junction laws" and 4 "loop laws" satisfied by the scalar currents $A_{i}$ (where Feynman parameters $z_{i}$ play the role of resistance) [35, 36]. Another type of modification was to reduce the number of integration variables by exploiting the fact that in some diagrams the integrand depends only on a particular combination of Feynman parameters. These resulted in substantial reduction in the size of integrands and the amount of computing time required to achieve desired precision. In the "new" version, those modifications were not employed at all because they are diagram-specific and not suitable for automation. As a result, the size of FORTRAN source code for $M_{01}$ (see Fig. 1), which

requires only vertex renormalization, is about $515 \mathrm{~KB}$ in the "new" version in contrast to 
$316 \mathrm{~KB}$ of the old version. A more notable difference is seen for the diagram $M_{12}$ which requires IR subtraction. The "new" $M_{12}$ occupies $630 \mathrm{~KB}$ while the old $M_{12}$ occupies only 21KB. As a consequence, the old and "new" integrals have much different forms so that they can be regarded to be independent of each other as far as numerical integration is concerned.

\section{Ultraviolet (UV) divergence}

The amplitude $M_{\mathcal{G}}$ has (logarithmic) UV-divergences in general. Suppose we want to find out whether $M_{\mathcal{G}}$ diverges when all loop momenta of a subdiagram $\mathcal{S}$ consisting of $N_{\mathcal{S}}$ lines and $n_{\mathcal{S}}$ closed loops go to infinity. In the parametric formulation this limit corresponds to the vanishing of $U$ when all $z_{i}$ for $i \in \mathcal{S}$ vanish simultaneously. To find the criterion for the UV divergence from $\mathcal{S}$, consider the part of the integration domain where $z_{i}$ for $i \in S$ satisfy $\sum_{i \in \mathcal{S}} z_{i} \leq \epsilon$. In the limit $\epsilon \rightarrow 0$ one finds [36, 37]

$$
\begin{aligned}
V & =\mathcal{O}(1), \quad U=\mathcal{O}\left(\epsilon^{n_{\mathcal{S}}}\right) \\
B_{i j} & = \begin{cases}\mathcal{O}\left(\epsilon^{n_{\mathcal{S}}-1}\right) & \text { for } i, j \in \mathcal{S} \\
\mathcal{O}\left(\epsilon^{n_{\mathcal{S}}}\right) & \text { otherwise. }\end{cases}
\end{aligned}
$$

The UV-divergent part can be identified by the following procedure called $K$-operation:

(a) In the limit (19) keep only terms with the lowest power of $\epsilon$ in $U, B_{i j}$, and $A_{i}$. In this limit $U$ factorizes as $U_{\mathcal{S}} U_{\mathcal{G} / \mathcal{S}}$ where $\mathcal{G} / \mathcal{S}$ is obtained from $\mathcal{G}$ by shrinking $\mathcal{S}$ to a point in G. $B_{i j}$ factorizes similarly. $V$ is reduced to $V_{\mathcal{G} / \mathcal{S}}$, where $V_{\mathcal{G} / \mathcal{S}}$ is the $V$ function defined on $\mathcal{G} / \mathcal{S}$.

(b) Replace $V_{\mathcal{G} / \mathcal{S}}$ by $V_{\mathcal{G} / \mathcal{S}}+V_{\mathcal{S}}$.

(c) Rewrite the integrand of $M_{\mathcal{G}}$ in terms of parametric functions redefined in (国) and (b)), and drop all terms except those with the largest number of contractions [35] within $\mathcal{S}$. The result is denoted by $\mathbb{K}_{\mathcal{S}} M_{\mathcal{G}}$, in which $\mathbb{K}_{\mathcal{S}}$ stands for an operator acting on $M_{\mathcal{G}}$.

By construction, $\mathbb{K}_{\mathcal{S}} M_{\mathcal{G}}$ has the same UV divergence as $M_{\mathcal{G}}$ in the same integration domain. Therefore it can be used as a pointwise subtraction term in the subtractive renormalization.

An important feature of $K$-operation is that the resulting integral can be factorized exactly into a product or a sum of products of lower-order quantities that consists of a leading 
UV-divergent part of the renormalization constant and the magnetic moment part. The $K$-operation associated with a UV-divergent subdiagram $\mathcal{S}$ produces, when $\mathcal{S}$ is of vertex type, the subtraction term of the form [36, 37]

$$
\mathbb{K}_{\mathcal{S}} M_{\mathcal{G}}=L_{\mathcal{S}}^{\mathrm{UV}} M_{\mathcal{G} / \mathcal{S}}
$$

where $L_{\mathcal{S}}^{\mathrm{UV}}$ is the leading UV-divergent part of the vertex renormalization constant $L_{\mathcal{S}}$ and $M_{\mathcal{G} / \mathcal{S}}$ is the magnetic moment part of the reduced diagram $\mathcal{G} / \mathcal{S}$. When $\mathcal{S}$ is a self-energy subdiagram, the $K$-operation yields [36, 37]

$$
\mathbb{K}_{\mathcal{S}} M_{\mathcal{G}}=\delta m_{\mathcal{S}}^{\mathrm{UV}} M_{\mathcal{G} / \mathcal{S}\left(i^{\star}\right)}+B_{\mathcal{S}}^{\mathrm{UV}} M_{\mathcal{G} /[\mathcal{S}, i]}
$$

where $\delta m_{\mathcal{S}}^{\mathrm{UV}}$ is the leading UV-divergent part of the mass-renormalization constant $\delta m_{\mathcal{S}}$, $B_{\mathcal{S}}^{\mathrm{UV}}$ is the leading UV-divergent part of the wave-function renormalization constant $B_{\mathcal{S}}$, and the reduced diagram $\mathcal{G} /[\mathcal{S}, i]$ is obtained from $\mathcal{G}$ by removing $\mathcal{S}$ and a lepton line $i$ adjacent to $\mathcal{S}$.

The whole UV-divergent structure of the amplitude $M_{\mathcal{G}}$ can be recognized by Zimmer-

mann's forest formula [14]. A forest is a set of UV-divergent subdiagrams in which any pair of subdiagrams is either disjoint (they do not share lines or vertices) or inclusive (one subdiagram is a subgraph of the other subdiagram). Each subtraction term corresponds to a forest. In our formulation, the subtraction term is obtained by successive application of $K$-operations for every element of the forest. The UV-finite amplitude $\underline{M}_{\mathcal{G}}$ created by $K$-operation is thus expressed in the form

$$
\underline{M}_{\mathcal{G}}=M_{\mathcal{G}}+\sum_{f} \prod_{\mathcal{S} \in f}\left(-\mathbb{K}_{\mathcal{S}}\right) M_{\mathcal{G}},
$$

where the summation is taken over the normal forests of the diagram $\mathcal{G}$ that do not include $\mathcal{G}$ itself as an element.

N. B. In both old and "new" approaches UV divergence is treated by the same K-operation.

\section{Infrared (IR) divergence}

A diagram may have an IR singularity when some of the internal photon momenta vanish. In order that this singularity becomes actually divergent, however, it must be enhanced by vanishing of denominators of two or more electron propagators (called enhancers) due to 
kinematical constraints. Such a situation occurs in diagrams that have self-energy-like subdiagrams. In Eq. (17) this corresponds to the vanishing of $V$-function of the denominators in the integration domain characterized by [36, 37]

$$
z_{i}= \begin{cases}\mathcal{O}(\delta) & \text { if } i \text { is an electron line in } \mathcal{R} \\ \mathcal{O}(1) & \text { if } i \text { is a photon line in } \mathcal{R} \\ \mathcal{O}(\epsilon), \quad \epsilon \sim \delta^{2}, & \text { if } i \in \mathcal{S},\end{cases}
$$

where $\mathcal{R}=\mathcal{G} / \mathcal{S}$.

This enables us to obtain a simple IR power-counting rule for identifying IR divergent terms. When there are two enhancers, the amplitude shows a logarithmic IR divergence. We can identify and construct the corresponding subtraction term by the following procedure called I-operation [36, 37]:

(a) In the limit (23) keep only terms with lowest power of $\epsilon$ and $\delta$ in $U, B_{i j}, A_{i}$. The numerator then factorizes to the product

$$
F \rightarrow F_{\mathcal{R}} F_{\mathcal{S}},
$$

where $F_{\mathcal{R}}$ is a numerical factor obtained by replacing all scalar currents $A_{i}$ in the diagram $\mathcal{R}$ by one.

(b) Make the following replacements:

$$
U \rightarrow U_{\mathcal{S}} U_{\mathcal{R}}, \quad V \rightarrow V_{\mathcal{S}}+V_{\mathcal{R}}, \quad F \rightarrow F_{0}\left[L_{\mathcal{R}}\right] F_{\mathcal{S}},
$$

where $F_{0}\left[L_{\mathcal{R}}\right]$ is the no-contraction part of the vertex renormalization constant defined in $\mathcal{R}$. The difference between $F_{0}\left[L_{\mathcal{R}}\right]$ and $F_{\mathcal{R}}$ causes a finite difference of the integration.

(c) Rewrite the integrand of $M_{\mathcal{G}}$ in terms of redefined parametric functions, keeping only the IR-divergent terms.

In the "old" method all logarithmic IR divergences have been subtracted by means of the I-operation. However, the case involving linear IR divergence, which has three enhancers, was handled by an ad hoc manner instead of a systematic approach. Actually, the cause of linear IR-divergence is easy to identify. It is caused by our treatment of self-energy subdiagram by means of $K$-operation which subtracts only the UV-divergent part of the 
self-mass. The unsubtracted part of self-mass keeps the number of enhancers unchanged, except in second-order case where the $K$-operation subtracts the self-mass term completely.

In the "new" approach, a systematic method is developed to handle the linear IR divergence. To remove the finite remnant of self-mass term completely, an $R$-subtraction operation [15] is newly introduced. After the $R$-subtraction operation is carried out, which decreases the number of enhancers to two, only logarithmic IR divergences remain, which can be handled by the $I$-subtraction operation similar to, but different in detail from, the I-operation of the "old" method.

For a formal treatment, we introduce two operators for these subtractions. The $R$ subtraction operator $\mathbb{R}_{\mathcal{S}}$ acts as

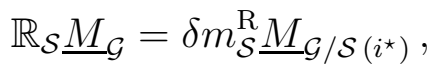

where $\delta m_{\mathcal{S}}^{\mathrm{R}}$ is the residual part of the mass renormalization constant defined by

$$
\delta m_{\mathcal{S}}^{\mathrm{R}}=\delta m_{\mathcal{S}}-\delta m_{\mathcal{S}}^{\mathrm{UV}}+\sum_{f} \prod_{\mathcal{S}^{\prime} \in f}\left(-\mathbb{K}_{\mathcal{S}^{\prime}}\right) \widetilde{\delta m_{\mathcal{S}}}
$$

in which the leading UV-divergent part $\delta m_{\mathcal{S}}^{\mathrm{UV}}$ and the subdivergent parts associated with the forests $\prod_{\mathcal{S}^{\prime} \in f}\left(-\mathbb{K}_{\mathcal{S}^{\prime}}\right) \widetilde{\delta m_{\mathcal{S}}}$ are subtracted away, where $\widetilde{\delta m} \equiv \delta m-\delta m^{\mathrm{UV}}$.

The $I$-subtraction operator $\mathbb{I}_{\mathcal{S}}$ acts on the UV-renormalized amplitude $\underline{M}_{\mathcal{G}}$ as

$$
\mathbb{I}_{\mathcal{S}} \underline{M}_{\mathcal{G}}=L_{\mathcal{G} / \mathcal{S}}^{\mathrm{R}} \underline{M}_{\mathcal{S}}
$$

where $L_{\mathcal{G} / \mathcal{S}}^{\mathrm{R}}$ is the residual part of the vertex renormalization constant defined by

$$
L_{\mathcal{G} / \mathcal{S}}^{\mathrm{R}}=L_{\mathcal{G} / \mathcal{S}}-L_{\mathcal{G} / \mathcal{S}}^{\mathrm{UV}}+\sum_{f} \prod_{\mathcal{S}^{\prime} \in f}\left(-\mathbb{K}_{\mathcal{S}^{\prime}}\right) \widetilde{L}_{\mathcal{G} / \mathcal{S}}
$$

in which the leading UV-divergent part $L_{\mathcal{G} / \mathcal{S}}^{\mathrm{UV}}$ and the subdivergent parts associated with the forests $\prod_{\mathcal{S}^{\prime} \in f}\left(-\mathbb{K}_{\mathcal{S}^{\prime}}\right) \widetilde{L}_{\mathcal{G} / \mathcal{S}}$ are subtracted away, where $\widetilde{L} \equiv L-L^{\mathrm{UV}}$.

$N$. B. The IR power counting rule identifies only IR-divergent terms. It does not specify how to handle IR-finite term. The "new" I-subtraction operation handles the IR-finite terms differently from the "old" I-operation. The I-subtraction operation needs not deal with the IR divergence associated with a vertex subdiagram of the self-energy-like diagram, while the $\mathfrak{I}$-operation directly acts on the vertex subdiagram.

The whole set of IR subtraction terms can be obtained by the combinations of these two operations, both of which belong to annotated forests [15]. An annotated forest is a set of 
self-energy-like subdiagrams, to each element of which the distinct operation of $I$-subtraction or $R$-subtraction is assigned. The IR-subtraction term associated with an annotated forest is constructed by successively applying operators $\mathbb{I}$ or $\mathbb{R}$, and takes the form

$$
\left(-\mathbb{I}_{\mathcal{S}_{i}}\right) \ldots\left(-\mathbb{R}_{\mathcal{S}_{j}}\right) \ldots \underline{M}_{\mathcal{G}}
$$

where the annotated forest $\widetilde{f}$ consists of the subdiagrams $\mathcal{S}_{i}, \ldots$ and $\mathcal{S}_{j}, \ldots$

$N$. B. The IR divergence is treated differently in the old and "new" approaches. This difference plays an important part in ensuring the independence of two calculations.

\section{E. Residual renormalization}

Because of difference in the handling of IR divergences in the "old" and "new" methods, we obtain different forms of residual renormalization. Since the "old" residual renormalization is described in Refs. [36, 37], let us consider here only the "new" residual renormalization.

In the "new" approach the UV- and IR-finite amplitude has the form

$$
\Delta M_{\mathcal{G}}=M_{\mathcal{G}}+\sum_{f} \prod_{\mathcal{S} \in f}\left(-\mathbb{K}_{\mathcal{S}}\right) M_{\mathcal{G}}+\sum_{\tilde{f}}\left(-\mathbb{I}_{\mathcal{S}_{i}}\right) \cdots\left(-\mathbb{R}_{\mathcal{S}_{j}}\right) \cdots \underline{M}_{\mathcal{G}},
$$

where $\underline{M}_{\mathcal{G}}$ is the UV-finite quantity defined by Eq. (22). $\Delta M_{\mathcal{G}}$ can be readily turned into a numerical integration code by $\operatorname{GENCODE} N[14,15]$ and is to be evaluated by numerical means.

This procedure is different from the standard on-shell renormalization which is defined by the on-shell quantities. The difference between the on-shell quantities and the quantity evaluated by Eq. (31) must be compensated by products of known lower-order quantities. We call this step the residual renormalization. See Appendix B for details.

\section{EIGHTH-ORDER TERMS}

The eighth-order term $A_{1}^{(8)}$ receives contributions from 891 Feynman diagrams. The 373 of them have closed lepton loops and had been evaluated by two or more independent methods [16]. The remaining 518 diagrams of $q$-type form one gauge-invariant set (Group V). In our approach they are represented by 47 independent diagrams shown in Fig.1 by using 


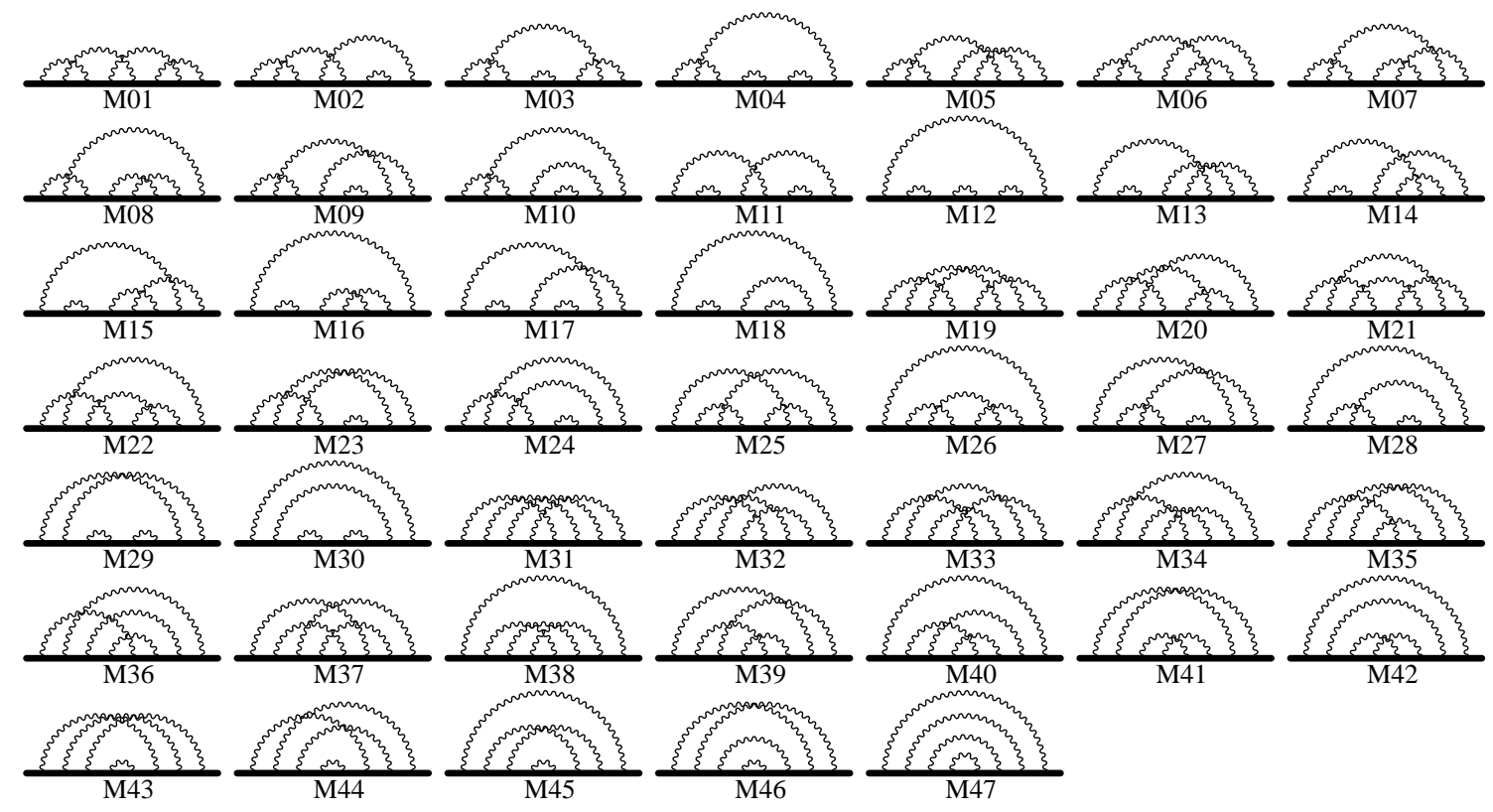

FIG. 1: Eighth-order Group V diagrams represented by 47 self-energy-like diagrams $M_{01}-M_{47}$.

the relation derived from Ward-Takahashi identity and the time-reversal symmetry. Thus far, there is only one complete evaluation of the eighth-order term, which was performed by numerical means [16]. Some of these diagrams have linear IR divergence, which was treated by an ad hoc subtraction method. In contrast GENCODE $N$ is capable of dealing with such hard IR divergence in a systematic fashion [15]. The application of GENCODE $N$ to the calculation of the eighth-order $q$-type diagrams provides us the opportunity not only to test if it works properly, but also to check the previous result.

Even in the eighth-order case GENCODE $N$ creates FORTRAN programs very rapidly. The entire 47 program sets are generated in less than ten minutes on hp's Alpha. The numerical evaluation is, however, quite non-trivial and requires a huge computational resource. For the preliminary evaluation we have used 64 to 256 Xeon CPU's per diagram and run the programs over a few months. To our surprise it uncovered an inconsistency in the treatment of IR subtraction terms in the old calculation. In Secs. [II] and IV] we describe how this inconsistency was uncovered by a detailed comparison of the old code and the code generated by GENCODE $N$. 


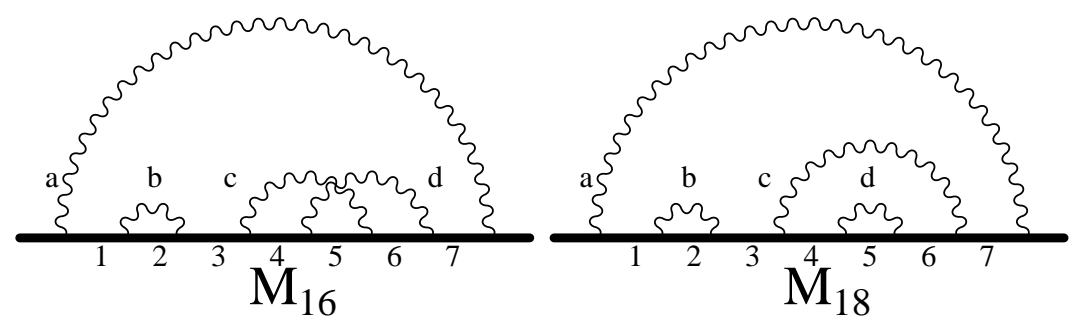

FIG. 2: Self-energy-like diagrams of $M_{16}$ and $M_{18}$. Feynman parameters assigned to the electron lines are $z_{1} \sim z_{7}$ and those to the photon lines are $z_{a} \sim z_{d}$.

\section{A. IR treatments of the eighth-order diagrams}

The treatments of IR subtraction terms are different in GENCODE $N$ and the "old" approach. The difference of IR subtraction terms leads to the difference of the finite part of the amplitude $\Delta M_{i}(i=01, \cdots, 47)$. The difference $\Delta M_{i}^{\text {old }}-\Delta M_{i}^{\text {new }}$ between the old amplitude $\Delta M_{i}^{\text {old }}$ and the "new" one $\Delta M_{i}^{\text {new }}$ is finite and can be expressed analytically in terms of finite lower-order quantities. We will see if this difference is numerically reproduced by substituting the numerical values calculated separately for these lower-order quantities. If the numerical discrepancy is found, there must be something wrong in either the "old" or "new" calculation. This is what we tried to find out.

We noted in Sec. IIC that the subtraction of UV divergences is achieved by the same $K$-operation in both GENCODE $N$ and "old" calculation. Therefore, the difference between $\Delta M_{i}^{\text {old }}$ and $\Delta M_{i}^{\text {new }}$, if it exists, comes exclusively from the difference of IR subtraction procedures between the "new" and "old" calculations. To examine this difference more closely let us begin by considering relatively simple diagrams which contain only one secondorder self-energy subdiagram. Diagrams belonging to this class are $M_{02}, M_{03}, M_{09}, M_{13}$, $M_{14}, M_{15}, M_{23}, M_{24}, M_{27}, M_{43}, M_{44}$. As an example let us consider the diagram $M_{14}$.

The IR divergence occurs in $M_{14}$ from the second-order self-energy subdiagram which consists of an electron line "2" and a photon line "b" in Fig. 3(a). In the W-T summed diagram this subdiagram plays dual roles. One part of this subdiagram behaves as a genuine self-mass term and the associated UV singularity is removed completely by the $\mathbb{K}_{2}$-operation. Another part works as the second-order magnetic moment $M_{2}$, and the residual diagram surrounding $M_{2}$ behaves like a sixth-order vertex diagram $L_{6 g 5}$ of Fig. 3(b), which is IRdivergent. 


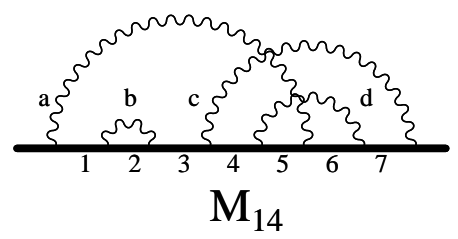

(a)

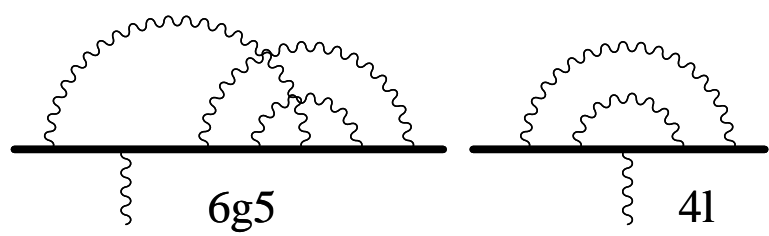

(b)

FIG. 3: (a) Self-energy-like diagram of $M_{14}$. (b)Vertex diagrams $6 g 5$ and $4 l$ from which the vertex renormalization constants $L_{6 g 5}$ and $L_{4 l}$, respectively, are derived.

In the "old" approach, the finite contribution $\Delta M_{14}$ was defined by

$$
\Delta M_{14}^{\mathrm{old}} \equiv M_{14}+\sum_{f} \prod_{\mathcal{S} \in f}\left(-\mathbb{K}_{\mathcal{S}}\right) M_{14}-\left(I_{6 g 5}+I_{2} \Delta L_{4 l}\right) M_{2}
$$

Here the second term on the right-hand side is the sum of UV subtraction terms given by the K-operation. The last two terms beginning with the letter "I" are the IR subtraction terms generated by the $\mathfrak{I}$-operations $\mathfrak{I}_{134567}$ and $\mathfrak{I}_{13}\left(1-\mathfrak{I}_{134567}\right)$ in the "old" approach. They arise from the "magnetic-moment part" of the self-energy-like subdiagram mentioned above. Note that they are exactly identical with the IR-divergent parts of $L_{6 g 5}$ :

$$
L_{6 g 5} \equiv I_{6 g 5}+I_{2} \Delta L_{4 l}+\Delta L_{6 g 5}-\sum_{f} \prod_{\mathcal{S} \in f}\left(-\mathbb{K}_{\mathcal{S}}\right) \widetilde{L}_{6 g 5}+L_{6 g 5}^{\mathrm{UV}}
$$

Here the sum appearing on the right-hand side denotes all the UV subdivergences contained in $L_{6 g 5}$ (whose explicit form is $-L_{2}^{U V} \widetilde{L}_{4 c}-L_{4 l}^{U V} \widetilde{L}_{2}+\left(L_{2}^{U V}\right)^{2} \widetilde{L}_{2}$ ), and the last term is the overall UV divergence of $L_{6 g 5}$.

In the "new" (or GENCODE $N$ ) approach, we introduce a term $L_{6 g 5}^{\mathrm{R}}$ defined by

$$
L_{6 g 5}^{\mathrm{R}} \equiv L_{6 g 5}-L_{6 g 5}^{\mathrm{UV}}+\sum_{f} \prod_{\mathcal{S} \in f}\left(-\mathbb{K}_{\mathcal{S}}\right) \widetilde{L}_{6 g 5}
$$

and $\Delta M_{14}^{\text {new }}$ by

$$
\Delta M_{14}^{\text {new }}=\underline{M}_{14}-\mathbb{I}_{2} \underline{M}_{14}
$$

where $\mathbb{I}_{2}$ is an $I$-subtraction operation associated with the self-energy-like subdiagram $\mathcal{S}=$ $\{2, b\}$, and yields

$$
\mathbb{I}_{2} \underline{M}_{14}=L_{6 g 5}^{\mathrm{R}} M_{2}
$$


From Eqs.(33) and (34) we obtain

$$
L_{6 g 5}^{\mathrm{R}}=I_{6 g 5}+I_{2} \Delta L_{4 l}+\Delta L_{6 g 5}
$$

This is UV-finite and consists of not only IR divergent terms but also a completely finite term $\Delta L_{6 g 5}$. It follows that $\Delta M_{14}^{\text {old }}$ and $\Delta M_{14}^{\text {new }}$ differ by

$$
\Delta M_{14}^{\text {old }}-\Delta M_{14}^{\text {new }}=\Delta L_{6 g 5} M_{2}
$$

Since $\Delta L_{6 g 5}$ is UV- and IR-finite, it can be computed without encountering with UV or IR divergence. This is true for every $\Delta M_{i}^{\text {old }}-\Delta M_{i}^{\text {new }}$ as it originates from the choice of finite pieces that accompany the singular terms. The choice adopted in $\Delta M_{14}^{\text {new }}$ turned out to be preferred since it leads to a simpler formula and can be readily extended to other cases.

All eleven diagrams listed above can be analyzed in the same manner. The diagrams $M_{04}$, $M_{11}, M_{12}, M_{17}, M_{29}, M_{30}$, which contain two or three second-order self-energy-like subdiagrams, are slightly more complicated, but can be treated in a similar manner. Evaluation of the diagrams with one self-energy subdiagram of fourth- or sixth-order such as $M_{08}, M_{10}$, $M_{26}, M_{38}, M_{40}, M_{41}$ is more complicated and needs the residual self-mass renormalization, the $R$-subtraction, as well as the $I$-subtraction. But they do not present particular difficulty as far as IR subtraction is concerned. (See Appendix B for more information on these diagrams.)

The diagrams $M_{28}, M_{42}, M_{45}, M_{46}, M_{47}$ are even more complicated due to nested structure, but they can also be handled by slight extensions. (See Appendix B.)

The most difficult of the eighth-order $q$-type diagrams are those containing one secondorder self-energy-like subdiagram and one fourth-order self-energy-like subdiagram, namely, $M_{16}$ and $M_{18}$ of Fig. 2. The difficulty originates from the fact that these diagrams have linear IR divergence. Detailed analysis of these diagrams is deferred to Sec. IV.

\section{B. Numerical result of eighth-order calculation}

We present the results of our numerical study for $\Delta M_{i}^{\text {old }}-\Delta M_{i}^{\text {new }}$ in Tables 【, II and III. In these tables, the second columns list the analytic expression of $\Delta M_{i}^{\text {old }}-\Delta M_{i}^{\text {new }}$ in terms of finite pieces of lower-order renormalization constants and magnetic moment amplitudes multiplied by the multiplicity, which is 1 for the time-reversal-symmetric diagram and 2 
TABLE I: Comparison of the numerical calculation of $M_{01}-M_{15}$ of the eighth-order Group V diagrams. The second column shows the analytic expression for $\Delta M_{i}^{\text {old }}-\Delta M_{i}^{\text {new }}$ for each diagram $M_{i}$ in terms of lower-order finite quantities multiplied by the multiplicity. The value $A$ in the third column is obtained by substituting the values of lower-order renormalization constants, such as $\Delta M_{4 a}, \Delta L_{4 s}$ and $\Delta M_{4 a\left(1^{\star}\right)}$, for the corresponding expression in the second column. See Appendices B and $\mathrm{C}$ for the detail. In contrast, the value for $\Delta M_{i}^{\text {old }}-\Delta M_{i}^{\text {new }}$ in the fourth column, denoted by value $B$, is obtained by taking the direct difference between the value of $\Delta M_{i}^{\text {old }}$ quoted from Ref. [16], and the one of $\Delta M_{i}^{\text {new }}$ calculated via GENCODE $N$ in the "new" IR subtraction procedure [14, 15]. The fifth column lists up the differences $A-B$. If the whole calculation is done correctly, $A-B$ must vanish within the numerical uncertainty. In evaluating $\Delta M^{\text {new }}$ the double precision is used for the diagrams without a self-energy subdiagram, while the quadruple precision is used for the remainder.

\begin{tabular}{llccc}
\hline \hline Diagram & difference & value $A$ & value $B$ & $A-B$ \\
\hline$M_{01}$ & 0 & 0 & $-0.0129(47)$ & $0.0129(47)$ \\
$M_{02}$ & $2 \Delta L_{6 f 1} M_{2}$ & $-0.0063(2)$ & $0.0060(110)$ & $-0.0124(110)$ \\
$M_{03}$ & $\Delta L_{6 f 3} M_{2}$ & $-0.1133(1)$ & $-0.1055(100)$ & $-0.0078(100)$ \\
$M_{04}$ & $2\left(\Delta L_{6 d 1}+\Delta L_{6 d 3}\right) M_{2}$ & $0.3350(2)$ & $0.3408(175)$ & $-0.0058(175)$ \\
$M_{05}$ & 0 & 0 & $0.0020(28)$ & $-0.0020(28)$ \\
$M_{06}$ & 0 & 0 & $-0.0223(61)$ & $0.0223(61)$ \\
$M_{07}$ & 0 & 0 & $-0.0102(40)$ & $0.0102(40)$ \\
$M_{08}$ & $2\left(\Delta \delta m_{4 a} \Delta M_{4 a\left(1^{\star}\right)}+\Delta L_{4 c} \Delta M_{4 a}\right)$ & $-2.1809(7)$ & $-2.1790(121)$ & $-0.0019(121)$ \\
$M_{09}$ & $2 \Delta L_{6 f 2} M_{2}$ & $0.0806(1)$ & $0.0894(109)$ & $-0.0088(109)$ \\
$M_{10}$ & $2\left(\Delta \delta m_{4 b} \Delta M_{4 a\left(1^{\star}\right)}+\Delta L_{6 d 2} M_{2}+\Delta L_{4 c} \Delta M_{4 b}\right)$ & $15.8898(49)$ & $15.8795(147)$ & $0.0103(155)$ \\
$M_{11}$ & $2 \Delta L_{6 d 5} M_{2}$ & $0.6949(2)$ & $0.6827(112)$ & $0.0122(112)$ \\
$M_{12}$ & $\left(2 \Delta L_{6 a 1}+\Delta L_{6 a 3}\right) M_{2}$ & $1.2842(0)$ & $1.2875(74)$ & $-0.0034(74)$ \\
$M_{13}$ & $2 \Delta L_{6 h 1} M_{2}$ & $-0.4211(2)$ & $-0.4238(48)$ & $0.0027(48)$ \\
$M_{14}$ & $2 \Delta L_{6 g 5} M_{2}$ & $0.0892(2)$ & $0.0960(95)$ & $-0.0068(95)$ \\
$M_{15}$ & $2 \Delta L_{6 g 1} M_{2}$ & $0.0883(2)$ & $0.0893(71)$ & $-0.0009(71)$ \\
\hline \hline
\end{tabular}


TABLE II: Comparison of the numerical calculations of $M_{16}-M_{30}$ of the eighth-order Group V diagrams.

\begin{tabular}{clrrr}
\hline \hline Diagram & \multicolumn{1}{c}{ difference } & value $A$ & value $B$ & $A-B$ \\
\hline$M_{16}$ & $2\left(\Delta \delta m_{4 a} \Delta M_{4 b\left(1^{\star}\right)}+\Delta L_{6 c 1} M_{2}+\Delta L_{4 s} \Delta M_{4 a}\right)$ & $-2.6042(6)$ & $-2.6316(235)$ & $0.0274(235)$ \\
$M_{17}$ & $2\left(\Delta L_{6 e 1}+\Delta L_{6 d 4}\right) M_{2}$ & $-2.1201(2)$ & $-2.1010(189)$ & $-0.0173(189)$ \\
$M_{18}$ & $2\left\{\Delta \delta m_{4 b} \Delta M_{4 b\left(1^{\star}\right)}+\Delta L_{4 s} \Delta M_{4 b}\right.$ & $16.9686(39)$ & $17.1897(206)$ & $-0.2207(210)$ \\
& $\left.+\left(\Delta L_{6 b 1}+\Delta L_{6 a 2}\right) M_{2}\right\}$ & & & \\
$M_{19}$ & 0 & & & \\
$M_{20}$ & 0 & 0 & $0.0002(3)$ & $-0.0002(3)$ \\
$M_{21}$ & 0 & 0 & $0.0010(17)$ & $-0.0010(17)$ \\
$M_{22}$ & 0 & 0 & $0.0003(3)$ & $-0.0003(3)$ \\
$M_{23}$ & $2 \Delta L_{6 h 2} M_{2}$ & 0 & $-0.0090(25)$ & $0.0090(25)$ \\
$M_{24}$ & $2 \Delta L_{6 g 2} M_{2}$ & $0.0501(2)$ & $0.0438(59)$ & $0.0064(59)$ \\
$M_{25}$ & 0 & $0.0789(2)$ & $0.0945(61)$ & $-0.0155(61)$ \\
$M_{26}$ & $\Delta \delta m_{6 f}\left(M_{2^{\star}}-M_{2^{\star}}[I]\right)$ & 0 & $-0.0031(20)$ & $0.0031(20)$ \\
$M_{27}$ & $2 \Delta L_{6 g 4} M_{2}$ & $-0.2857(2)$ & $-0.2809(109)$ & $-0.0048(109)$ \\
$M_{28}$ & $2\left\{\Delta \delta m_{6 d}\left(M_{2^{\star}}-M_{2^{\star}}[I]\right)+\Delta L_{6 c 2} M_{2}\right\}$ & $-7.5332(5)$ & $-7.5307(153)$ & $-0.0025(153)$ \\
$M_{29}$ & $2 \Delta L_{6 e 2} M_{2}$ & $0.2763(6)$ & $0.2675(153)$ & $0.0088(153)$ \\
$M_{30}$ & $\Delta \delta m_{6 a}\left(M_{2^{\star}}-M_{2^{\star}}[I]\right)+2 \Delta L_{6 b 2} M_{2}$ & & & \\
\hline \hline & & $2.5119(3)$ & $2.5369(95)$ & $-0.0250(95)$ \\
\hline
\end{tabular}

otherwise. Each value in the third columns, called "value A", is obtained by substituting the values of these renormalization constants, etc., listed in Table IV, for the corresponding expression in the second columns.

In contrast to value $\mathrm{A}$, each value in the fourth columns, called "value B", is obtained by taking the difference between the numerical value $\Delta M_{i}^{\text {old }}$ quoted from the literature [16] and the one $\Delta M_{i}^{\text {new }}$ newly calculated via GENCODE $N$ according to the "new" IR subtraction procedure [15]. The fifth columns list up the difference of value A and value B for each $i$, denoted by $A-B$. It must be zero within numerical precision if the whole calculation has been done correctly. If value $\mathrm{A}$ and value $\mathrm{B}$ are different, there are two possible sources. One possibility is that the program used for a numerical calculation has a bug. It means that either $\Delta M_{i}^{\text {old }}$ or $\Delta M_{i}^{\text {new }}$ is wrong, or both are wrong. The other possibility is that we 
TABLE III: Comparison of the numerical calculations of $M_{31}-M_{47}$ of the eighth-order Group V diagrams.

\begin{tabular}{|c|c|c|c|c|}
\hline Diagram & difference & value $A$ & value $B$ & $A-B$ \\
\hline$M_{31}$ & 0 & 0 & $0.0007(5)$ & $-0.0007(5)$ \\
\hline$M_{32}$ & 0 & 0 & $-0.0024(10)$ & $0.0024(10)$ \\
\hline$M_{33}$ & 0 & 0 & $0.0001(3)$ & $-0.0001(3)$ \\
\hline$M_{34}$ & 0 & 0 & $-0.0010(13)$ & $0.0010(13)$ \\
\hline$M_{35}$ & 0 & 0 & $0.0001(13)$ & $-0.0001(13)$ \\
\hline$M_{36}$ & 0 & 0 & $-0.0027(22)$ & $0.0027(22)$ \\
\hline$M_{37}$ & 0 & 0 & $0.0004(5)$ & $-0.0004(5)$ \\
\hline$M_{38}$ & $\Delta \delta m_{6 h}\left(M_{2^{\star}}-M_{2^{\star}}[I]\right)$ & $-0.9088(3)$ & $-0.9112(40)$ & $0.0024(40)$ \\
\hline$M_{39}$ & 0 & 0 & $-0.0031(18)$ & $0.0031(18)$ \\
\hline$M_{40}$ & $2 \Delta \delta m_{6 g}\left(M_{2^{\star}}-M_{2^{\star}}[I]\right)$ & $3.8281(3)$ & $3.8326(71)$ & $-0.0045(71)$ \\
\hline$M_{41}$ & $\Delta \delta m_{4 a}\left(\Delta M_{4 a\left(2^{\star}\right)}\right)+\Delta L_{4 x} \Delta M_{4 a}$ & $0.9809(3)$ & $0.9713(83)$ & $0.0096(83)$ \\
\hline$M_{42}$ & $\begin{array}{l}\Delta \delta m_{6 c}\left(M_{2^{\star}}-M_{2^{\star}}[I]\right)+\Delta L_{4 l} \Delta M_{4 a} \\
+\Delta \delta m_{4 a}\left\{\Delta M_{4 b\left(2^{\star}\right)}-\Delta \delta m_{2^{\star}}\left(M_{2^{\star}}-M_{2^{\star}}[I]\right)\right\}\end{array}$ & $-7.0218(4)$ & $-7.0202(114)$ & $-0.0016(114)$ \\
\hline$M_{43}$ & $\Delta L_{6 h 3} M_{2}$ & $0.4724(1)$ & $0.4703(42)$ & $0.0022(42)$ \\
\hline$M_{44}$ & $2 \Delta L_{6 g 3} M_{2}$ & $-0.0748(1)$ & $-0.0499(69)$ & $-0.0250(69)$ \\
\hline$M_{45}$ & $\Delta \delta m_{6 e}\left(M_{2^{\star}}-M_{2^{\star}}[I]\right)+\Delta L_{6 c 3} M_{2}$ & $-0.0523(3)$ & $-0.0498(90)$ & $-0.0025(90)$ \\
\hline$M_{46}$ & $\Delta \delta m_{4 b} \Delta M_{4 a\left(2^{\star}\right)}+\Delta L_{6 e 3} M_{2}+\Delta L_{4 x} \Delta M_{4 b}$ & $-7.9339(22)$ & $-7.9232(86)$ & $-0.0107(89)$ \\
\hline$M_{47}$ & $\begin{array}{l}\Delta \delta m_{6 b}\left(M_{2^{\star}}-M_{2^{\star}}[I]\right)+\Delta L_{6 b 3} M_{2}+\Delta L_{4 l} \Delta M_{4 b} \\
+\Delta \delta m_{4 b}\left\{\Delta M_{4 b\left(2^{\star}\right)}-\Delta \delta m_{2^{\star}}\left(M_{2^{\star}}-M_{2^{\star}}[I]\right)\right\}\end{array}$ & $10.5872(15)$ & $10.5864(102)$ & $0.0008(103)$ \\
\hline
\end{tabular}

incorrectly identified the analytic difference between the "old" and "new" methods.

For a diagram $M_{i}$ without any self-energy-like subdiagrams, the analytic expression of $\Delta M_{i}^{\text {old }}-\Delta M_{i}^{\text {new }}$ is trivially zero, as it does not have IR divergence. We can see from the corresponding values B in Tables $\amalg$, II and $\amalg$ III that this is confirmed within the numerical precision employed.

The diagrams containing self-energy-like subdiagrams suffer from IR divergence. Tables \, II and III show that "old" and "new" calculations are in good agreement for most of these 
TABLE IV: Finite renormalization constants used in Table I, II, and III. Sixth-order vertex renormalization constants are shown in this table. Their validity is checked by comparing the sum $X_{L B D} \equiv \sum_{i=1}^{5} \Delta L_{6 x i}+\frac{1}{2} \Delta B_{6 x}+2 \Delta \delta m_{6 x}, \quad x=a, \cdots h$ to the previous $X_{L B D}$ values listed in Ref. [16].

\begin{tabular}{lccccc}
\hline \hline$\Delta L_{6 a 1}$ & $0.539604(45)$ & $\Delta L_{6 a 2}$ & $-0.167211(81)$ & $\Delta L_{6 a 3}$ & $1.489159(98)$ \\
$\Delta L_{6 b 1}$ & $-1.479745(109)$ & $\Delta L_{6 b 2}$ & $0.582944(106)$ & $\Delta L_{6 b 3}$ & $-0.016344(73)$ \\
$\Delta L_{6 c 1}$ & $-0.219365(98)$ & $\Delta L_{6 c 2}$ & $0.071504(87)$ & $\Delta L_{6 c 3}$ & $-0.552261(107)$ \\
$\Delta L_{6 d 1}$ & $0.834949(96)$ & $\Delta L_{6 d 2}$ & $-0.090796(92)$ & $\Delta L_{6 d 3}$ & $-0.499995(97)$ \\
$\Delta L_{6 d 4}$ & $-1.378190(109)$ & $\Delta L_{6 d 5}$ & $0.694916(101)$ & & \\
$\Delta L_{6 e 1}$ & $-0.741904(144)$ & $\Delta L_{6 e 2}$ & $-0.285670(108)$ & $\Delta L_{6 e 3}$ & $-0.141787(122)$ \\
$\Delta L_{6 f 1}$ & $-0.006322(114)$ & $\Delta L_{6 f 2}$ & $0.080648(97)$ & $\Delta L_{6 f 3}$ & $-0.226693(106)$ \\
$\Delta L_{6 g 1}$ & $0.088204(70)$ & $\Delta L_{6 g 2}$ & $0.078922(103)$ & $\Delta L_{6 g 3}$ & $-0.074834(92)$ \\
$\Delta L_{6 g 4}$ & $-0.062995(85)$ & $\Delta L_{6 g 5}$ & $0.089213(69)$ & & \\
$\Delta L_{6 h 1}$ & $-0.421132(108)$ & $\Delta L_{6 h 2}$ & $0.050140(108)$ & $\Delta L_{6 h 3}$ & $0.944887(116)$ \\
$\Delta \delta m_{6 a}$ & $-0.15331(26)$ & $\Delta \delta m_{6 b}$ & $1.83795(19)$ & $\Delta \delta m_{6 c}$ & $-3.05047(17)$ \\
$\Delta \delta m_{6 d}$ & $-1.90117(11)$ & $\Delta \delta m_{6 e}$ & $0.11193(13)$ & $\Delta \delta m_{6 f}$ & $1.25594(10)$ \\
$\Delta \delta m_{6 g}$ & $0.95702(6)$ & $\Delta \delta m_{6 h}$ & $-0.45441(5)$ & & \\
\hline \hline
\end{tabular}

diagrams. However, a large discrepancy $-0.221(21)$ is found for the diagram $M_{18}$. Though no detectable discrepancy is found for $M_{16}$, it has a structure similar to $M_{18}$ and is somewhat simpler to analyze. In Section IV we thus look for the origin of such a discrepancy through a detailed investigation of $M_{16}$.

\section{DETAILED EXAMINATION OF $M_{16}$}

In the "old" approach the finite contribution $\Delta M_{16}$ was given by [36, 38]

$$
\begin{aligned}
\Delta M_{16}^{\text {old }} & \equiv M_{16}+\sum_{f} \prod_{\mathcal{S} \in f}\left(-\mathbb{K}_{\mathcal{S}}\right) M_{16} \\
& -I_{6 c 1} M_{2}-\frac{1}{2} J_{6 c} M_{2}-I_{4 s} \Delta M_{4 a}-\Delta \delta m_{4 a} I_{4 b\left(1^{\star}\right)}+I_{2^{\star}} \Delta \delta m_{4 a} M_{2},
\end{aligned}
$$


TABLE V: Finite renormalization constants used in Table I, II and III. Fourth-order and secondorder quantities are given here.

\begin{tabular}{llll}
\hline \hline$\Delta L_{4 c}$ & $0.003387(16)$ & $\Delta L_{4 x}$ & $-0.481834(54)$ \\
$\Delta L_{4 s}$ & $0.407633(20)$ & $\Delta L_{4 l}$ & $0.124796(67)$ \\
$\Delta B_{4 a}$ & $-0.039811(15)$ & $\Delta B_{4 b}$ & $-0.397283(15)$ \\
$\Delta \delta m_{4 a}$ & $-0.301485(61)$ & $\Delta \delta m_{4 b}$ & $2.20777(44)$ \\
$\Delta M_{4 a}$ & $0.218359(39)$ & $\Delta M_{4 b}$ & $-0.187526(39)$ \\
$\Delta M_{4 a\left(1^{\star}\right)}$ & $3.6192(31)$ & $\Delta M_{4 a\left(2^{\star}\right)}$ & $-3.6003(19)$ \\
$\Delta M_{4 b\left(1^{\star}\right)}$ & $\Delta .2486(15)$ & $\Delta M_{4 b\left(2^{\star}\right)}$ & $1.6432(15)$ \\
$\Delta M_{2}$ & 0.5 & $\Delta M_{2^{\star}}$ & 1 \\
$\Delta M_{2^{\star}}[I]$ & -1 & $\Delta \delta m_{2^{\star}}$ & -0.75 \\
$\Delta B_{2}$ & 0.75 & $\Delta B_{2^{\star}}[I]$ & -0.5 \\
$\Delta L_{2^{\star}}$ & -0.75 & $\Delta B_{2^{\star}}$ & 1.5 \\
\hline \hline
\end{tabular}

while the "new" version is given by

$$
\begin{aligned}
\Delta M_{16}^{\text {new }} & \equiv M_{16}+\sum_{f} \prod_{\mathcal{S} \in f}\left(-\mathbb{K}_{\mathcal{S}}\right) M_{16} \\
& -L_{6 c 1}^{\mathrm{R}} M_{2}-L_{4 s}^{\mathrm{R}} \Delta M_{4 a}-\Delta \delta m_{4 a} \underline{M}_{4 b\left(1^{\star}\right)}+L_{2^{\star}}^{\mathrm{R}} \Delta \delta m_{4 a} M_{2},
\end{aligned}
$$

where $L_{2^{\star}}^{\mathrm{R}}=I_{2^{\star}}$. Note that " $2^{\star}$ " denotes the second-order diagram with a two-point vertex inserted into the internal lepton line. " $4 b\left(1^{\star}\right)$ " denotes the diagram obtained from the fourth-order diagram $4 b$ by inserting a two-point vertex into the lepton line 1 .

\section{A. Unrenormalized amplitude and UV subtraction terms of $M_{16}$}

We began our examination by comparing the unrenormalized amplitude $M_{16}$ and its UV subtraction terms in the "old" and "new" programs. For this purpose we used the "spotcheck" method, by which the values of "old" and "new" integrands are compared at the same set of numerical values of integration variables. The integrand of $M_{16}$ is defined in the Feynman parameter space that spans a hyperplane in 11-dimensional space satisfying

$$
z_{1}+z_{2}+\cdots+z_{7}+z_{a}+z_{b}+z_{c}+z_{d}=1
$$


In the "new" version, this hyperplane is mapped onto a unit 10-dimensional hypercube. On the other hand, in the "old" version the integration space is mapped onto an 8-dimensional hypercube, since the integrand depends only on the combination of Feynman parameters $z_{137}=z_{1}+z_{3}+z_{7}$. To carry out the spot check, we must use the same mappings, so we changed the mapping of the "new" integrand to the "old" one defining $z_{1}=z_{3}=z_{7}=$ $(1 / 3) z_{137}$. In practice the set of input parameters is chosen from the neighborhood of the singular point of interest where numerical disagreement is likely to be magnified. But points too close to the singular point are avoided, where the noise due to round-off error obscures the meaningful information. The "old" integrals and "new" integrals of the unrenormalized term and UV subtraction terms should be algebraically equivalent but have different forms because of extensive simplification of the "old" integrands by means of various relations among scalar currents. The "spot-check" comparison of "old" and "new" unrenormalized and UV integrands proves unambiguously that they have nevertheless the same values within the precision of numerical evaluation. (Typically more than 10 digits in 14 digits precision.)

\section{B. IR subtraction terms of $M_{16}$}

The "spot-check" method, however, is not directly applicable for comparison of "old" and "new" IR subtraction terms, because they are algebraically different by construction. For this purpose we need to understand precisely the analytic structure of the IR subtraction terms in both "old" and "new" methods. Thus we follow an alternative approach by which we can identify how they differ from each other in the analytic form.

In the "old" method, the IR singularities of $M_{16}$ are isolated by the $\mathfrak{I}_{\mathcal{R}}$-operations, where $\mathcal{R}=\{1,3,7, a\},\{1,2,3,7, a, b\}$, or $\{1,3,4,5,6,7, a, c, d\}$. Thus, the formal expression of the IR-free contribution of $M_{16}$ is given by [37]

$$
\overline{\Delta M_{16}} \equiv\left(1-\mathfrak{I}_{137}\right)\left(1-\mathfrak{I}_{134567}\right)\left(1-\mathfrak{I}_{1237}\right) \underline{M}_{16},
$$

where $\underline{M}_{16}$ is the UV-finite amplitude obtained by the $K$-operations. The product

$\mathfrak{I}_{134567} \mathfrak{I}_{1237}$ gives no contribution, since they overlap each other and cannot take these IR limits simultaneously.

Following the "old" prescription in Ref. [37], individual IR subtraction terms of Eq. (42) 
can be written as follows:

$$
\begin{aligned}
& -\mathfrak{I}_{134567 \underline{M_{16}}}=-I_{6 c 1} M_{2}, \\
& -\mathfrak{I}_{1237} \underline{M}_{16}=-I_{4 c} \Delta M_{4 a}-M_{4 b\left(1^{\star}\right)}[I] \Delta \delta m_{4 a}, \\
& -\mathfrak{I}_{137}\left(1-\mathfrak{I}_{134567}\right)\left(1-\mathfrak{I}_{1237}\right) \underline{M}_{16}=+I_{2^{\star}} \Delta \delta m_{4 a} M_{2} .
\end{aligned}
$$

$I_{6 c 1}$ and $I_{4 c}$ are the no-contraction terms of the vertex renormalization constant $L_{6 c 1}$ and $L_{4 c}$, respectively. $M_{4 b\left(1^{\star}\right)}$ is the magnetic moment amplitude, which is obtained from the fourth-order diagram $M_{4 b}$ with the two-point vertex inserted into the fermion line 1 . Its argument $[I]$ implies that the numerator of the no-contraction term of $M_{4 b\left(1^{\star}\right)}$ is replaced by that of the vertex renormalization constant $L_{4 s}$, while discarding the contraction terms.

If there were only logarithmic IR divergence, $\overline{\Delta M}_{16}$ defined in (42) would be IR-finite, but it is not. The problem here is that $I_{6 c 1}$ and $M_{4 b\left(1^{\star}\right)}[I]$ in Eqs. (43) and (44) have linear IR divergence. The $\mathfrak{I}$-operation prescription is constructed so that it only deals with the leading IR singularity. Of all eighth-order $q$-type diagrams, $M_{16}$ and $M_{18}$ have the linear IR divergence. Since these are the only cases, we chose to deal with their next-to-leading-order IR divergences by an ad hoc method rather than constructing a general rule.

In the $\mathfrak{I}_{134567}$ limit of Eq. (43), the diagram $M_{16}$ decouples into the vertex diagram $L_{6 c 1}$ which consists of lepton lines 1,3,4,5,6,7 and photon lines $a, c, d$ and the magnetic moment part $M_{2}$ which consists of lepton line 2 and photon line $b$. All IR singularities originate from the vertex diagram $L_{6 c 1}$. The no-contraction term $L_{6 c 1}\left[F_{0}\right]$, namely $I_{6 c 1}$, includes the leading linear IR singularity as well as the next-to-leading logarithmic singularity.

The logarithmic IR singularity also arises from the $\mathfrak{I}_{137}$-limit of the one-contraction term $L_{6 c 1}\left[F_{1}\right]$. To deal with this, we constructed the quantity $J_{6 c}^{\text {unrenorm. }}$ in which the numerator is the $\mathfrak{I}_{137}$ limit of $L_{6 c 1}\left[F_{1}\right]$, but the denominator $V$ and $U$ is the same as $L_{6 c 1}$. The UV divergences of $J_{6 c}^{\text {unrenorm. }}$ are removed by the $\mathbb{K}_{456}, \mathbb{K}_{56}, \mathbb{K}_{45}$-operations:

$$
\begin{gathered}
\frac{1}{2} J_{6 c}=\left(1-\mathbb{K}_{456}\right)\left(1-\mathbb{K}_{45}\right)\left(1-\mathbb{K}_{56}\right)\left(-\frac{1}{32} \int(d z)_{G / S} \frac{f_{1}}{U^{3} V^{2}}\right), \\
f_{1}=-16\left[B_{45}\left(2-A_{6}\right)+2 B_{46}\left(1-2 A_{5}\right)+B_{56}\left(2-A_{4}\right)\right],
\end{gathered}
$$

where $S=\{2, b\}$.

Next we consider the $\mathfrak{I}_{1237}$-limit of Eq. (44). In this limit, the self-energy-like subdiagram consisting of lepton lines 4,5,6 and photon lines $c, d$ plays dual roles. When this fourthorder self-energy-like subdiagram behaves as a magnetic moment $M_{4 a}$, the residual diagram 
resembles a vertex diagram $L_{4 s}$. Its singularity is logarithmic, so $\mathfrak{I}_{1237}$-operation properly works for this part.

The problem arises when the self-energy-like subdiagram acts as the self-mass $\delta m_{4 a}$, which is the second one of its dual roles. The residual diagram is the magnetic moment amplitude with one two-point vertex inserted, namely, $M_{4 b\left(1^{\star}\right)}$. The power counting shows that it has a linear IR singularity. Thus, the $\mathfrak{I}$-operation is not enough to remove the IR singularity of this term. $M_{4 b\left(1^{\star}\right)}[I]$ is not sufficient to remove all IR singularities arising in the $\mathfrak{I}_{1237}$ limit. The IR structure of $M_{4 b\left(1^{\star}\right)}$ is more closely scrutinized in the next subsection, where $I_{4 b\left(1^{\star}\right)}$ is constructed to include both linear and logarithmic IR singularities of the magnetic moment amplitude $M_{4 b\left(1^{\star}\right)}$. (A similar subtraction method works also for $M_{18}$.)

Taking these considerations into account, we replace the IR subtraction terms of $M_{16}$ in the "old" method listed in Eqs. (43) and (44) with [36, 38]

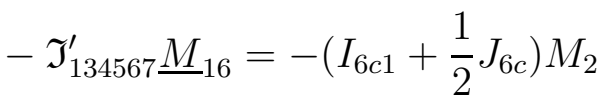

$$
\begin{aligned}
& -\mathfrak{I}_{1237}^{\prime} \underline{M}_{16}=-I_{4 c} \Delta M_{4 a}-I_{4 b\left(1^{\star}\right)} \Delta \delta m_{4 a}
\end{aligned}
$$

which are more convenient for comparison with the "new" approach. Note that Eq.(45) is unchanged.

Now, let us look at the "new" approach. All IR singularities, both linear and logarithmic, are subtracted by using the general rule applicable to any order of the perturbation theory. The $R$ - and $I$-subtractions, and their combinations determine the IR subtraction terms of $M_{16}$ as follows:

$$
\begin{aligned}
& -\mathbb{I}_{2} \underline{M}_{16}=-L_{6 c 1}^{\mathrm{R}} M_{2}, \\
& -\mathbb{I}_{456} \underline{M}_{16}=-L_{4 s}^{\mathrm{R}} \underline{M}_{4 a}, \\
& -\mathbb{R}_{456} \underline{M}_{16}=-\delta m_{4 a}^{\mathrm{R}} \underline{M}_{4 b\left(1^{\star}\right)}, \\
& +\mathbb{I}_{2} \mathbb{R}_{456} \underline{M}_{16}=+L_{2^{\star}}^{\mathrm{R}} \delta m_{4 a}^{\mathrm{R}} M_{2} .
\end{aligned}
$$


By definition given in Eqs. (27) and (29), the residual quantities are explicitly given by

$$
\begin{aligned}
L_{6 c 1}^{\mathrm{R}} & =\left(1-\mathbb{K}_{456}\right)\left(1-\mathbb{K}_{45}\right)\left(1-\mathbb{K}_{56}\right)\left(L_{6 c 1}-L_{6 c 1}^{U V}\right) \\
& =L_{6 c 1}-L_{6 c 1}^{U V}-\left(B_{4 a}^{U V} \widetilde{L}_{2}^{\prime}+\delta m_{4 a}^{U V} L_{2^{\star}}\right)-2 L_{2}^{U V} \widetilde{L}_{4 s}+2 L_{2}^{U V}\left(\delta m_{2} L_{2^{\star}}+B_{2}^{U V} \widetilde{L}_{2}^{\prime}\right), \\
L_{4 s}^{\mathrm{R}} & =\left(1-\mathbb{K}_{2}\right)\left(L_{4 s}-L_{4 s}^{U V}\right)=L_{4 s}-L_{4 s}^{U V}-\left(B_{2}^{U V} \widetilde{L}_{2}^{\prime}+\delta m_{2} L_{2^{\star}}\right), \\
\underline{M}_{4 a} & =\left(1-\mathbb{K}_{45}\right)\left(1-\mathbb{K}_{56}\right) M_{4 a}=M_{4 a}-2 L_{2}^{U V} M_{2}, \\
\delta m_{4 a}^{\mathrm{R}} & =\delta m_{4 a}-\delta m_{4 a}^{U V} \\
\underline{M}_{4 b\left(1^{\star}\right)} & =\left(1-\mathbb{K}_{2}\right) M_{4 b\left(1^{\star}\right)}=M_{4 b\left(1^{\star}\right)}-\left(B_{2}^{U V} M_{2^{\star}}+\delta m_{2} M_{2^{\star \star}}\right),
\end{aligned}
$$

where $\widetilde{L}=L-L^{U V}$. (See below Eq. (27).) In terms of the "old" expression of the unrenormalized amplitude and renormalization constants, the residual quantities are related to the IR divergent and finite pieces of the "old" method by the following relations:

$$
\begin{aligned}
& L_{6 c 1}^{\mathrm{R}}=I_{6 c 1}+\frac{1}{2} J_{6 c}+\Delta L_{6 c 1}, \\
& L_{4 s}^{\mathrm{R}}=I_{4 s}+\Delta L_{4 s}, \\
& \underline{M}_{4 a}=\Delta M_{4 a}, \\
& \delta m_{4 a}^{\mathrm{R}}=\Delta \delta m_{4 a}, \\
& \underline{M}_{4 b\left(1^{\star}\right)}=I_{4 b\left(1^{\star}\right)}+\Delta M_{4 b\left(1^{\star}\right)} .
\end{aligned}
$$

We are now ready to compare the IR subtraction terms of "old" and "new" method side by side:

$$
\begin{array}{lc}
\quad \text { old } & \text { new } \\
-\left(I_{6 c 1}+\frac{1}{2} J_{6 c}\right) M_{2} & -\left(I_{6 c 1}+\frac{1}{2} J_{6 c}+\Delta L_{6 c 1}\right) M_{2} \\
-I_{4 s} \Delta M_{4 a} & -\left(I_{4 s}+\Delta L_{4 s}\right) \Delta M_{4 a} \\
-I_{4 b\left(1^{\star}\right)} \Delta \delta m_{4 a} & -\left(I_{4 b\left(1^{\star}\right)}+\Delta M_{4 b\left(1^{\star}\right)}\right) \Delta \delta m_{4 a} \\
+I_{2^{\star}} \Delta \delta m_{4 a} M_{2} & +I_{2^{\star}} \Delta \delta m_{4 a} M_{2} .
\end{array}
$$

Actually, instead of examining the IR subtraction terms of the "old" method themselves we reconstructed them from the "new" programs by dropping finite terms (eg. $\Delta L_{4 s}$ ) from the "Residual" term (eg. $L_{4 s}^{\mathrm{R}}$ ), and compared them with the terms in the "old" programs by the spot-check method. To obtain $I_{6 c 1}$ and $I_{4 s}$, we only need to comment out the contraction terms (equivalently drop the terms proportional to $B_{i j}$ ) of $L_{6 c 1}^{\mathrm{R}}$ and $L_{4 s}^{\mathrm{R}}$ of the "new" programs. In this way, we found that the reconstructed IR subtraction terms from the "new" programs are identical with "old" ones for (a), (b), and (d). 
However, the "old" IR subtraction term (c) $I_{4 b\left(1^{\star}\right)} \Delta \delta m_{4 a}$ cannot be constructed by such a simple recipe from the "new" programs generated by GenCode $N$. Dropping the finite terms in $\underline{M}_{4 b\left(1^{\star}\right)} \Delta \delta m_{4 a}$ is not enough to reproduce $I_{4 b\left(1^{\star}\right)} \Delta \delta m_{4 a}$. Therefore, we reconstructed the subtraction term $I_{4 b\left(1^{\star}\right)} \Delta \delta m_{4 a}$ from the scratch using the definitions of the fourth-order quantities $I_{4 b\left(1^{\star}\right)}$ and $\Delta \delta m_{4 a}$. Then, the result is compared with the integrand in the "old" program of $\Delta M_{16}^{\text {old }}$.

\section{C. $I_{4 b\left(1^{\star}\right)} \Delta \delta m_{4 a}$ by the "old" I-operation}

Let us first explain how $I_{4 b\left(1^{\star}\right)} \Delta \delta m_{4 a}$ is obtained in the "old" program. In the old approach, the IR subtraction term $I_{4 b\left(1^{\star}\right)} \Delta \delta m_{4 a}$ originates from the $\mathfrak{I}_{1237}$-operation. In addition to this term, $\mathfrak{I}_{1237}$ operation yields the term $I_{4 s} \Delta M_{4 a}$.

The IR-limit associated with the operator $\mathfrak{I}_{1237}$ is given by

$$
z_{a}+z_{b}=1-\mathcal{O}(\delta), \quad z_{1}, z_{2}, z_{3}, z_{7}=\mathcal{O}(\delta), \quad z_{4}, z_{5}, z_{6}, z_{c}, z_{d}=\mathcal{O}(\epsilon), \quad \epsilon \sim \delta^{2}, \quad \delta \rightarrow 0
$$

In the neighborhood of this limit we have $A_{1}=1-\mathcal{O}(\delta), A_{2}=1-\mathcal{O}(\delta)$, and $V=$ $\mathcal{O}\left(\delta^{2}\right)$. As is discussed in the previous section, the result of $\mathfrak{I}_{1237}$-operation includes both linear and logarithmic IR divergences. In particular, the linear divergence is associated with $\mathfrak{I}_{137} \mathfrak{I}_{1237}$ limit. If we apply the $\mathfrak{I}_{137} \mathfrak{I}_{1237}$ operation, however, it subtracts the linear divergence correctly, but not the logarithmic divergence. Thus, we chose an ad hoc method in which the piece including linear divergence is separated out from the result of $\mathfrak{I}_{1237}$-operation and put aside for a while. The remainder that contains only logarithmic divergence is named $f_{k}$. The linear divergent piece is redefined so that it is defined on the subdiagram $\{1,2,3,7, a, b\}$ without decomposing it into two subdiagrams $\{2, b\}$ and $\{1,3,7, a\}$, which occurs in the naïve $\mathfrak{I}_{137} \mathfrak{I}_{1237}$ limit. This term is named $f_{l}$. The explicit forms of $f_{k}$ and $f_{l}$ in the old FORTRAN program of $\Delta M_{16}$ read:

$$
\begin{gathered}
f_{k}=\int(d z)_{\mathcal{G}} \frac{1}{4 U^{2}} L_{4 s}\left[F_{0}\right]\left(\frac{E_{0}+C_{0}+\delta m_{4 a}\left[f_{0}\right]+g_{\mathcal{S}} F_{1}+Y_{1}}{V^{3}}+\frac{3\left(g_{\mathcal{S}}-V_{t}\right) \delta m_{4 a}\left[f_{0}\right]+Y_{0}}{V^{4}}\right), \\
f_{l}=-\frac{3}{2} \int(d z)_{\mathcal{G}} \frac{\delta m_{4 a}\left[f_{0}\right]}{U^{2} V^{4}} z_{2}\left(1-A_{2}\right)^{2}\left(-1+6 A_{1}-3 A_{1}^{2}+2 A_{1}^{3}\right),
\end{gathered}
$$


where

$$
\begin{aligned}
L_{4 s}[ & \left.F_{0}\right]=\left(4 A_{1}-2 A_{2}\right)\left(1-A_{1}+A_{1}^{2}\right)+\left(-2+A_{1} A_{2}\right)\left(1-4 A_{1}+A_{1}^{2}\right) \\
F_{1}= & B_{45}\left(2-A_{6}\right) / U+2 B_{46}\left(1-2 A_{5}\right) / U+B_{56}\left(2-A_{4}\right) / U \\
Y_{1}= & -z_{4}\left(B_{45}\left(1-A_{6}\right)+B_{46}+B_{56} A_{4}\right) / U \\
& +z_{5}\left(B_{45}\left(1-A_{6}\right)-4 B_{46} A_{5}+B_{56}\left(1-A_{4}\right)\right) / U \\
& \quad-z_{6}\left(B_{45} A_{6}+B_{46}+B_{56}\left(1-A_{4}\right)\right) / U \\
V_{t}= & z_{137}\left(1-A_{1}\right)-z_{2}\left(1-A_{2}\right) \\
E_{0}= & 2 A_{4} A_{5} A_{6}-A_{4} A_{5}-A_{4} A_{6}-A_{5} A_{6} \\
C_{0}= & -3 z_{c} z_{d} / U_{\mathcal{S}} \\
& \\
\delta m_{4 a}[ & \left.f_{0}\right]=E_{0}+1-2 A_{5} \\
Y_{0}= & z_{4}\left(-A_{4}+A_{5}+A_{6}+A_{4} A_{5}+A_{4} A_{6}-A_{5} A_{6}\right) \\
& +z_{5}\left(1-A_{4} A_{5}+A_{4} A_{6}-A_{5} A_{6}+2 A_{4} A_{5} A_{6}\right) \\
& +z_{6}\left(A_{4}+A_{5}-A_{6}-A_{4} A_{5}+A_{4} A_{6}+A_{5} A_{6}\right) \\
g_{\mathcal{S}}= & z_{4} A_{4}+z_{5} A_{5}+z_{6} A_{6} .
\end{aligned}
$$

The building blocks $U, V, A_{i}, B_{i j}$ of the above integrands are obtained from those for $M_{16}$ by taking the IR-limit associated with the $\mathfrak{I}_{1237}$-operation. Recall that in the IR-limit the subdiagram $\mathcal{S}$ consisting of the fermion lines 4,5,6 and photon lines $c, d$, and the reduced diagram $\mathcal{G} / \mathcal{S}$ consisting of the fermion lines $1,2,3,7$ and the photon lines $a, b$ decouple from each other. Thus, the building blocks are actually the same as those obtained by taking the 
UV-limit associated with the $\mathbb{K}_{456}$ limit. Their explicit forms are

$$
\begin{aligned}
& U=U_{\mathcal{S}} U_{\mathcal{G} / \mathcal{S}} \\
& U_{\mathcal{S}}=z_{46 c d} z_{5}+z_{4 c} z_{6 d}, \quad U_{\mathcal{G} / \mathcal{S}}=z_{137 a} z_{2 b}+z_{2} z_{b} \\
& V=V_{\mathcal{S}}+V_{\mathcal{G} / \mathcal{S}} \\
& V_{\mathcal{S}}=z_{456}-z_{4} A_{4}-z_{5} A_{5}-z_{6} A_{6}+\lambda^{2} z_{c d}, \quad V_{\mathcal{G} / \mathcal{S}}=z_{1237}-z_{137} A_{1}-z_{2} A_{2}+\lambda^{2} z_{a b}, \\
& A_{i}=1-\sum_{j=1}^{7} z_{i} B_{i j} / U, \quad i=1, \cdots 7 \\
& B_{11}=B_{13}=B_{17}=B_{33}=B_{37}=B_{37}=z_{2 b} U_{\mathcal{S}} \\
& B_{45}=z_{6 d} U_{\mathcal{G} / \mathcal{S}}, \quad B_{46}=-z_{5} U_{\mathcal{G} / \mathcal{S}}, \quad B_{56}=z_{4 c} U_{\mathcal{G} / \mathcal{S}} \\
& B_{i j}=0 \quad \text { for } i \in \mathcal{S} \text { and } j \in \mathcal{G} / \mathcal{S} .
\end{aligned}
$$

In the above $z_{i_{1} i_{2} \ldots}$ stands for $z_{i_{1}}+z_{i_{2}}+\cdots$ and the electron mass is taken as a unit of mass scale (i.e., 1) and the photon mass is $\lambda$. In the leading order of the $\mathfrak{I}_{1237}$ limit $L_{4 s}\left[F_{0}\right]$ tends to 4 . The actual form of $L_{4 s}\left[F_{0}\right]$ in Eq. (56) was chosen so that the integral $f_{k}$ decouples into known lower-order quantities. This difference for $L_{4 s}\left[F_{0}\right]$ is IR-finite. Note that $\delta m_{4 a}\left[f_{0}\right]$ is related to the integrand of $\Delta \delta m_{4 a}$, namely, the UV-and IR-finite part of the mass renormalization constant $\delta m_{4 a}$ given later in Eq. (65).

In order to clarify the structure of $f_{k}$ and $f_{l}$, let us split $f_{k}$ into the three parts $f_{k 1}, f_{k 2}, f_{k 3}$,

$$
\begin{aligned}
f_{k 1} & =\int(d z)_{\mathcal{G}} \frac{1}{4 U^{2}} L_{4 s}\left[F_{0}\right]\left(\frac{E_{0}+C_{0}+g_{\mathcal{S}} F_{1}+Y_{1}}{V^{3}}+\frac{3 g_{\mathcal{S}} \delta m_{4 a}\left[f_{0}\right]+Y_{0}}{V^{4}}\right), \\
f_{k 2} & =\int(d z)_{\mathcal{G}} \frac{1}{4 U^{2}} L_{4 s}\left[F_{0}\right] \delta m_{4 a}\left[f_{0}\right]\left(\frac{1}{V^{3}}-\frac{3 z_{137}\left(1-A_{1}\right)}{V^{4}}\right) \\
f_{k 3} & =\int(d z)_{\mathcal{G}} \frac{1}{4 U^{2}} L_{4 s}\left[F_{0}\right] \delta m_{4 a}\left[f_{0}\right] \frac{3 z_{2}\left(1-A_{2}\right)}{V^{4}}
\end{aligned}
$$

and $f_{l}$ into the two parts $f_{l 1}, f_{l 2}$

$$
\begin{aligned}
f_{l 1} & =\frac{3}{4} \int(d z)_{\mathcal{G}} \frac{\delta m_{4 a}\left[f_{0}\right]}{U^{2} V^{4}} z_{2} A_{2}\left(1-A_{2}\right)\left(-1+6 A_{1}-3 A_{1}^{2}+2 A_{1}^{3}\right), \\
f_{l 2} & =-\frac{3}{4} \int(d z)_{\mathcal{G}} \frac{\delta m_{4 a}\left[f_{0}\right]}{U^{2} V^{4}} z_{2}\left(1-A_{2}\right)\left(2-A_{2}\right)\left(-1+6 A_{1}-3 A_{1}^{2}+2 A_{1}^{3}\right) .
\end{aligned}
$$

The integrand $f_{k 1}$ was compared with the integrand $\mathbb{I}_{456} M_{16}$ generated by GENCODE $N$ by the spot-check method. We confirmed that the integral $f_{k 1}$ minus its $\mathbb{K}_{2}$ limit, $f_{k v}$, is equal to $I_{4 s} \Delta M_{4 a}$, which is listed as (b) of (52). It turns out that $f_{k 2}+f_{l 1}$ is equal to $I_{4 b\left(1^{\star}\right)} \Delta \delta m_{4 a}$, 
which is reconstructed from the lower order quantities in the next subsection. Thus, the difference between the "old" calculation and the reconstructed one is confined in $f_{k 3}+f_{l 2}$. It is IR-finite but contributes a nonzero value to $\Delta M_{16}^{\text {old }}$.

\section{D. $I_{4 b\left(1^{\star}\right)} \Delta \delta m_{4 a}$ reconstruction from the lower-order quantities}

In order to understand where this difference $f_{k 3}+f_{l 2}$ came from, let us examine IR divergence structure of mass-inserted magnetic moment amplitude $M_{4 b\left(1^{\star}\right)}$ in the "old" approach. $I_{4 b\left(1^{\star}\right)}$ is defined from $M_{4 b\left(1^{\star}\right)}$ as follows [36, 38]:

$$
\begin{aligned}
M_{4 b\left(1^{\star}\right)}= & \left(1-\mathbb{K}_{2}\right)\left(1-\mathbb{I}_{a l l}\right)\left(M_{4 b\left(1^{\star}\right)}-M_{4 b\left(1^{\star}\right)}[f]\right)+\mathbb{K}_{2} M_{4 b\left(1^{\star}\right)} \\
& +\mathbb{I}_{\text {all }}\left(1-\mathbb{K}_{2}\right) M_{4 b\left(1^{\star}\right)}\left[N_{0}+Z_{0}-f, E_{0}+C_{0}\right]+M_{4 b\left(1^{\star}\right)}[f] \\
\equiv & \Delta M_{4 b\left(1^{\star}\right)}+\left(\delta m_{2} M_{2^{\star \star}}+B_{2}^{U V} M_{2^{\star}}\right)+I_{4 b\left(1^{\star}\right)} .
\end{aligned}
$$

The two terms in the second line of the r.h.s. define $I_{4 b\left(1^{\star}\right)}$ :

$$
I_{4 b\left(1^{\star}\right)} \equiv \mathbb{I}_{a l l}\left(1-\mathbb{K}_{2}\right) M_{4 b\left(1^{\star}\right)}\left[N_{0}+Z_{0}-f, E_{0}+C_{0}\right]+M_{4 b\left(1^{\star}\right)}[f]
$$

where the function $f$ is introduced in the first term in an ad hoc manner to subtract out the linear IR divergence coming from $N_{0}+Z_{0}$. The linear IR divergence is confined to the second term $M_{4 b\left(1^{\star}\right)}[f]$, which has the form

$$
\begin{aligned}
& M_{4 b\left(1^{\star}\right)}[f]=-\frac{1}{8} \int(d y)_{\mathcal{G} / \mathcal{S}} \frac{f}{U_{\mathcal{G} / \mathcal{S}}^{2} V_{\mathcal{G} / \mathcal{S}}^{3}}, \\
& f=-8 y_{2} A_{2}\left(1-A_{2}\right)\left(-1+6 A_{1}-3 A_{1}^{2}+2 A_{1}^{3}\right) .
\end{aligned}
$$

The explicit form of the first term of Eq.(61) is

$$
\mathbb{I}_{a l l} M_{4 b\left(1^{\star}\right)}\left[N_{0}+Z_{0}-f, E_{0}+C_{0}\right]=\lim _{\lambda \rightarrow 0} \int(d y)_{\mathcal{G} / \mathcal{S}} \frac{L_{4 s}\left[F_{0}\right]}{U_{\mathcal{G} / \mathcal{S}}^{2}}\left(\frac{1}{2 V_{\mathcal{G} / \mathcal{S}}^{2}}-\frac{y_{137}\left(1-A_{1}\right)}{V_{\mathcal{G} / \mathcal{S}}^{3}}\right)
$$

but i where

$$
\begin{aligned}
& V_{\mathcal{G} / \mathcal{S}}=y_{137}\left(1-A_{1}\right)+y_{2}\left(1-A_{2}\right)+\lambda^{2}\left(y_{a}+y_{b}\right) \\
& (d y)_{\mathcal{G} / \mathcal{S}}=d y_{1} d y_{2} d y_{3} d y_{7} d y_{a} d y_{b} \delta\left(1-y_{1237}-y_{a b}\right)
\end{aligned}
$$

The finite part of the mass renormalization constant $\Delta \delta m_{4 a}$ is

$$
\Delta \delta m_{4 a}=\frac{1}{4} \int(d y)_{\mathcal{S}} \frac{\delta m_{4 a}\left[f_{0}\right]}{U_{\mathcal{S}}^{2} V_{\mathcal{S}}}
$$


where $\delta m_{4 a}\left[f_{0}\right]$ is expressed in the same form in Eq. (56) and

$$
(d y)_{\mathcal{S}}=d y_{4} d y_{5} d y_{6} d y_{c} d y_{d} \delta\left(1-y_{456}-y_{c d}\right)
$$

Using the identity

$$
\frac{1}{V_{\mathcal{S}}^{m} V_{\mathcal{G} / \mathcal{S}}^{n}}=\frac{\Gamma(m+n)}{\Gamma(m) \Gamma(n)} \int_{0}^{1} d t \int_{0}^{1} d s \delta(1-t-s) \frac{t^{m-1} s^{n-1}}{\left(t V_{\mathcal{S}}+s V_{\mathcal{G} / \mathcal{S}}\right)^{m+n}}
$$

we can express the product of $\Delta \delta m_{4 a}$ and the first term of $I_{4 b\left(1^{\star}\right)}$ defined in Eq. (61) in the same Feynman parameter space as that for the original amplitude $M_{16}$

$$
\begin{aligned}
f_{k 2}^{\mathrm{rc}} & \equiv \Delta \delta m_{4 a} \times \mathbb{I}_{a l l} M_{4 b\left(1^{\star}\right)}\left[N_{0}+Z_{0}-f, E_{0}+C_{0}\right] \\
& =\int(d z)_{\mathcal{G}} \frac{L_{4 s}\left[F_{0}\right] \delta m_{4 a}\left[f_{0}\right]}{4 U^{2}}\left(\frac{1}{V^{3}}-\frac{3 z_{137}\left(1-A_{1}\right)}{V^{4}}\right),
\end{aligned}
$$

which is identical with $f_{k 2}$. Similarly, the contribution of the product of $\Delta \delta m_{4 a} M_{4 b\left(1^{\star}\right)}[f]$ to $M_{16}$ is

$$
\begin{aligned}
f_{l 1}^{\mathrm{rc}} & \equiv \Delta \delta m_{4 a} M_{4 b\left(1^{\star}\right)}[f] \\
& =\frac{3}{4} \int(d z)_{\mathcal{G}} \frac{\delta m_{4 a}\left[f_{0}\right]}{U^{2} V^{4}} z_{2} A_{2}\left(1-A_{2}\right)\left(-1+6 A_{1}-3 A_{1}^{2}+2 A_{1}^{3}\right),
\end{aligned}
$$

which is identical with $f_{l 1}$.

Therefore we find that the combination, $-f_{k 3}-f_{l 2}$, is extra in the "old" $\Delta M_{16}^{\text {old }}$ so that the correction term

$$
\begin{aligned}
\Delta M_{16}^{\text {add }} & \equiv 2\left(f_{k 3}+f_{l 2}\right) \\
& =-2 \times \frac{9}{4} \int(d z)_{\mathcal{G}} \frac{\delta m_{4 a}\left[f_{0}\right]}{U^{2} V^{4}} z_{2} A_{2}\left(1-A_{1}\right)^{3}\left(1-A_{2}\right),
\end{aligned}
$$

where the overall factor 2 comes from time-reversal diagram, must be added to $\Delta M_{16}^{\text {old }}$. Evaluating it numerically, we obtain $\Delta M_{16}^{\text {add }}=0.0294378$ (98), which is smaller than the current uncertainty of value $\mathrm{B}$ for $M_{16}$ in Table II and cannot be detected by the direct comparison of value A and value B until the latter is evaluated more precisely.

The difference between $\Delta M_{18}^{\text {new }}$ and $\Delta M_{18}^{\text {old }}$ can be analyzed in the same manner. It is found that the difference is numerically not small for $M_{18}$ :

$$
\begin{aligned}
\Delta M_{18}^{\text {add }} & \equiv 2\left(1-\mathbb{K}_{5}\right)\left(f_{k 3}+f_{l 2}\right) \\
& =-2 \times \frac{9}{4} \int(d z)_{\mathcal{G}}\left(1-\mathbb{K}_{5}\right)\left\{\frac{\delta m_{4 b}\left[f_{0}\right]}{U^{2} V^{4}} z_{2} A_{2}\left(1-A_{1}\right)^{3}\left(1-A_{2}\right)\right\} \\
& =-0.215542(19),
\end{aligned}
$$


where $A_{i}, U$, and $V$ are defined in the $\mathfrak{I}_{1237}$ limit of $M_{18}$. Of course their explicit forms are different from those of $M_{16}$. If we add $\Delta M_{18}^{\text {add }}$ to $\Delta M_{18}^{\text {old }}$, the value B for $M_{18}$ in Table II becomes 16.974 (21) and the difference between value A and value B is reduced to -0.006 (21), which is consistent with zero within the precision of numerical calculation.

\section{CONCLUSION}

The results described in this paper are summarized as follows:

1) There was an inconsistency between the "old" integrals $\Delta M_{16}^{\text {old }}$ and $\Delta M_{18}^{\text {old }}$ and their residual renormalization terms. This inconsistency is resolved in this paper.

2) Other 45 integrals of Group V of the "old" calculation are in good agreement with the "new" ones.

3) Programs generated by $\operatorname{Gencode} N$ have no error for $N=8$. Namely, the automation scheme has cleared the eighth-order test without difficulty.

The separation of the IR divergent and finite pieces in a given amplitude can be made arbitrarily. There is no overriding rule that dictates how to carry out such a separation. We only have to keep a record of what is subtracted as an IR subtraction term. All IR subtraction terms are summed up in the end and the arbitrariness in the choice of IR divergent part cancel out, leaving a finite contribution as a part of the residual renormalization.

The important point is that the IR subtraction term prepared for the numerical calculation and the one used to calculate the residual renormalization must be the same. What we found is that $I_{4 b\left(1^{\star}\right)}$ used in the numerical calculation of $M_{16}$ and $M_{18}$ and $I_{4 b\left(1^{\star}\right.}$ for the residual renormalization constant $\Delta M_{4 b\left(1^{\star}\right)}$ had different forms in the FORTRAN programs of the "old" calculation. This is the reason why $M_{16}$ and $M_{18}$ had IR-finite but redundant contributions.

The development of automatic code generator [14, 15] was crucial in enabling us to discover the existence of extra IR subtraction terms in $M_{16}$ and $M_{18}$ on short notice. Adding the correction terms $\Delta M_{16}^{\text {add }}$ and $\Delta M_{18}^{\text {add }}$ to the "old" value, we find the entire contribution of Group V to be

$$
A_{1}^{(8)}(\mathrm{GroupV})=-2.17916(343)
$$


which is in good agreement with the still preliminary value

$$
A_{1}^{(8)}(\mathrm{GroupV})=-2.219(53)
$$

obtained by the "new" code generated by GENCODE $N$.

Due to the different forms of IR subtraction terms, the forms of the residual renormalization are also different in the "old" and "new" calculations. The residual renormalization terms in the "old" IR procedure are given by [16, 36, 38]

$$
\begin{aligned}
A_{1}^{(8)}(\text { GroupV })^{\text {old }} & =\Delta M^{(8) \text { old }}-5 \Delta M^{(6) \text { old }} \Delta B_{2} \\
& -\Delta M^{(4)}\left\{4 \Delta L^{(4)}+3 \Delta B^{(4)}-9\left(\Delta B_{2}\right)^{2}\right\} \\
& -M_{2}\left\{2 \Delta L^{(6)}+\Delta B^{(6)}-\left(10 \Delta L^{(4)}+6 \Delta B^{(4)}\right) \Delta B_{2}+5\left(\Delta B_{2}\right)^{3}\right\} \\
& -\Delta M^{\left(4^{\star}\right)} \Delta \delta m^{(4)} \\
& -\left(M_{2^{\star}}-M_{2^{\star}}[I]\right)\left\{\Delta \delta m^{(6)}-\Delta \delta m^{(4)}\left(5 \Delta B_{2}+\Delta \delta m_{2^{\star}}\right)\right\} \\
& +M_{2} \Delta \delta m^{(4)}\left(4 \Delta L_{2^{\star}}+\Delta B_{2^{\star}}-B_{2^{\star}}[I]\right),
\end{aligned}
$$


where

$$
\begin{aligned}
& \Delta M^{(8) \text { old }}=\sum_{i=01}^{47} \Delta M_{i}^{\text {old }}, \\
& \Delta M^{(6) \text { old }}=\sum_{x=a}^{h} \Delta M_{6 x}^{\text {old }} \\
& \Delta M^{(4)}=\Delta M_{4 a}+\Delta M_{4 b}, \\
& \Delta M^{\left(4^{\star}\right)}=2 \Delta M_{4 a\left(1^{\star}\right)}+\Delta M_{4 a\left(2^{\star}\right)}+2 \Delta M_{4 b\left(1^{\star}\right)}+\Delta M_{4 b\left(2^{\star}\right)} \\
& \Delta L^{(6)}=\sum_{x=a}^{h} \sum_{i=1}^{5} \eta_{x} \Delta L_{6 x i} \\
& \Delta L^{(4)}=2 \Delta L_{4 c}+\Delta L_{4 x}+2 \Delta L_{4 s}+\Delta L_{4 l}, \\
& \Delta B^{(6)}=\sum_{x=a}^{h} \eta_{x} \Delta B_{6 x}, \\
& \Delta B^{(4)}=\Delta B_{4 a}+\Delta B_{4 b} \\
& \Delta \delta m^{(6)}=\sum_{x=a}^{h} \eta_{x} \Delta \delta m_{6 x}, \\
& \Delta \delta m^{(4)}=\Delta \delta m_{4 a}+\Delta \delta m_{4 b} \\
& \eta_{x}= \begin{cases}1 & \text { for } x=a, b, c, e, f, h \\
2 & \text { for } x=d, g .\end{cases}
\end{aligned}
$$

The numerical values of the finite renormalization constants are listed in Tables [V] and V], and also in Appendix [A], $B_{2^{\star}}[I]$ is obtained from the $\mathfrak{I}_{\text {all }}$-operation of the wave function renormalization constant $B_{2^{\star}}$.

The formula of the residual renormalization for the "new" calculation is much simpler than that for the "old" one. Since the mass renormalization is completed within the numerical calculation, the mass renormalization constant should not appear in the residual renormalization. The exceptions are the vertex and wave-function renormalization constants that have self-energy subdiagrams. The mass inserted vertex (wave-function) renormalization constant $L_{2^{\star}}\left(B_{2^{\star}}\right)$ has no overall UV divergence. As a result, the $K$-operation cannot pick up the renormalization terms proportional to $L_{2^{\star}}\left(B_{2^{\star}}\right)$. It must be restored in the residual renormalization in order to carry out the complete on-shell renormalization. The residual 
renormalization formula in the "new" approach is given by

$$
\begin{aligned}
A_{1}^{(8)}(\text { GroupV })^{\text {new }} & =\Delta M^{(8) \text { new }}-5 \Delta M^{(6) \text { new }} \Delta B_{2} \\
& -\Delta M^{(4)}\left\{3 \Delta L^{(4)}+3 \Delta B^{(4)}-9\left(\Delta B_{2}\right)^{3}\right\} \\
& -M_{2}\left\{\Delta L B^{(6)}-6\left(\Delta L^{(4)}+\Delta B^{(4)}\right) \Delta B_{2}+5\left(\Delta B_{2}\right)^{2}\right\} \\
& +M_{2} \Delta \delta m^{(4)}\left(4 \Delta L_{2^{\star}}+\Delta B_{2^{\star}}\right)
\end{aligned}
$$

where

$$
\begin{aligned}
& \Delta L B^{(6)}=\Delta L^{(6)}+\Delta B^{(6)}+\Delta L^{(4)} \Delta B_{2}+\Delta \delta m^{(4)} B_{2^{\star}}[I], \\
& \Delta M^{(6) \text { new }}=\Delta M^{(6) \text { old }}-\left(M_{2^{\star}}-M_{2^{\star}}[I]\right) \Delta \delta m^{(4)}-M_{2} \Delta L^{(4)} .
\end{aligned}
$$

In Eq. (76) the vertex renormalization constant $\Delta L^{(n)}$ and the wave function renormalization constant $\Delta B^{(n)}$ appear in the same weight for each order of the perturbation. It is because we have already subtracted one $\Delta L^{(n)}$ as an IR subtraction term. Calculating the combination $\Delta L^{(n)}+\Delta B^{(n)}$ is much easier than calculating each of them separately. Because of the Ward-Takahashi-identity for the renormalization constants $L^{(n)}+B^{(n)}=0$, many cancellations occur between two terms. Thus, we introduced a combined renormalization constant $\Delta L B^{(6)}$. Its relation to the "old" renormalization constants $\Delta L^{(6)}$ and $\Delta B^{(6)}$ are given in Eq. (77). More detailed definitions of $\Delta L B_{6 x}$ for each diagram are given in Appendix C3. The left-hand side of Eq. (177), $\Delta L B^{(6)}$, was directly calculated with the programs made by the automatic code generator for the residual renormalization constants [39] and obtained as

$$
\Delta L B^{(6)}=\sum_{x=a}^{h} \eta_{x} \Delta L B_{6 x}=0.10086(77) .
$$

This result was checked by comparing with the right-hand side of Eq. (177) calculated using the residual renormalization constant for the "old" calculation. The sixth-order magnetic moment $\Delta M^{(6) \text { new }}$ was calculated with the programs generated by GENCODE $N$ and given as

$$
\Delta M^{(6) \text { new }}=0.42610(53) .
$$

See Appendix $\mathrm{A} 2$ for the detail of $\Delta M^{(6) \text { new }}$.

As we have shown in the paper, the two results (72) and (73) of the eighth-order contribution from Group V diagrams are obtained by means of the totally independent calculations. 
Further theory corrections to the eighth-order term of the electron $g-2$ is very unlikely. The new theoretical prediction should be announced when we complete all the tenth-order calculation.

\section{Acknowledgments}

This work is partly supported by JSPS Grant-in-Aid for Scientific Research (C)19540322. M. H.'s work is also supported in part by JSPS and the French Ministry of Foreign Affairs under the Japan-France Integrated Action Program (SAKURA). T. K. 's work is supported by the U. S. National Science Foundation under Grant PHY-0355005. T. K. also thanks for JSPS Invitation Fellowship for Research in Japan S-07165, 2007. Numerical calculations were conducted on the RIKEN Super Combined Cluster System (RSCC).

\section{APPENDIX A: TEST OF GENCODEN BY LOWER-ORDER $a_{e}$}

Although GENCODE $N$ was developed primarily to deal with the tenth-order $q$-type diagrams, it can be readily applied to the calculation of fourth-, sixth-, and eighth-order $q$-type diagrams. Since these lower-order terms are also known from previous works, this serves for debugging of GENCODE $N$.

\section{Fourth-order $a_{e}$}

The fourth-order case is the simplest nontrivial example. The $g-2$ receives correction at this order from four types of vertex diagrams, $4 c, 4 x, 4 s$ and $4 l$ shown in Fig. 4(b). Following the remark in Sec. IA the sum of $g-2$ from the vertex diagrams $2 M_{4 c(s)}+M_{4 x(l)}$ is expressed as a quantity associated with a single self-energy diagram $M_{4 a(4 b)}$ in Fig. (4) (a) via the Ward-Takahashi identity (16) . (The factor 2 is assigned to the diagram to account for the presence of the diagram which is related by reversing the orientation of the lepton line.) GENCODE $N$ creates two program sets for $M_{4 a}$ and $M_{4 b}$ within a few seconds on a generic Linux PC. These programs give the finite amplitudes $\Delta M_{4 a(4 b)}$ as the sum of the unrenormalized Ward-Takahashi summed $g-2$ amplitudes, also denoted by $M_{4 a(4 b)}$, and necessary UV and/or IR subtraction terms. The relation of $\Delta M_{4 a(4 b)}$ to $M_{4 a(4 b)}$ is given in 


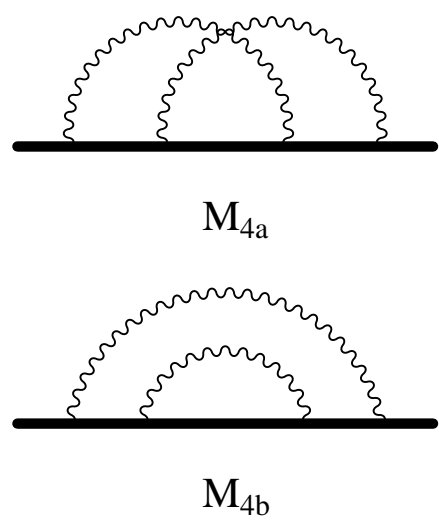

(a)
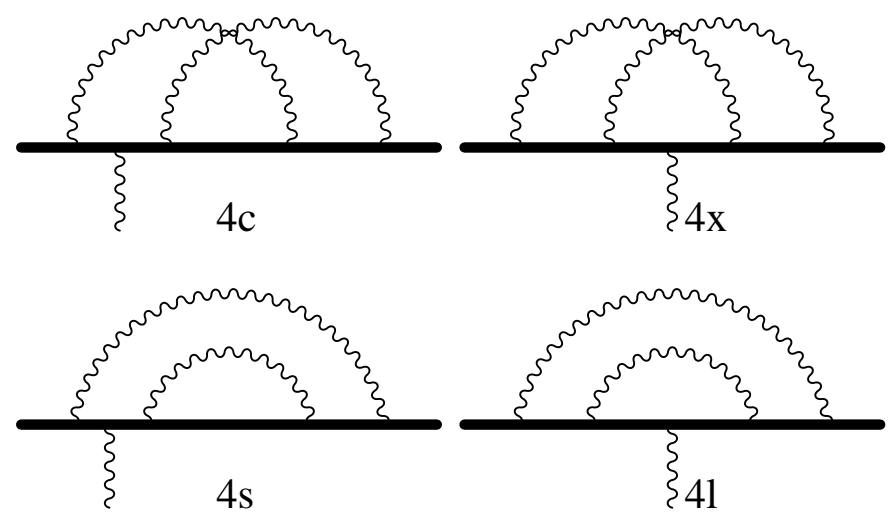

(b)

FIG. 4: Fourth-order $q$-type diagrams. (a) Self-energy-like diagrams $M_{4 a}$ and $M_{4 b}$. (b) Vertex diagrams $4 c, 4 x, 4 s$, and $4 l$. Their contributions to the magnetic moment are related to $M_{4 a}=$ $2 M_{4 c}+M_{4 x}$, and $M_{4 b}=2 M_{4 s}+M_{4 l}$.

Appendix B1. It took about 10 minutes each to carry out their numerical integration by VEGAS [17] with ten million sampling points per iteration for 50 iterations on hp's Alpha machine.

The values obtained in this way are

$$
\begin{aligned}
\Delta M_{4 a} & =0.21878(35), \\
\Delta M_{4 b} & =-0.18773(40) .
\end{aligned}
$$

The contribution from the fourth-order $q$-type diagrams is expressed as

$$
A_{1}^{(4)}(q \text {-type })=\Delta M_{4 a}+\Delta M_{4 b}-\Delta B_{2} M_{2}
$$

taking the residual renormalization into account. $M_{2}=1 / 2$ is the second-order correction to $g-2$ and $\Delta B_{2}=3 / 4$ is the finite part of the second-order wave function renormalization constant $B_{2}$. Substituting the numerical values (A1) for the formal expression (A2), we obtain

$$
A_{1}^{(4)}(q \text {-type })=-0.34395(53)
$$

which is in good agreement with the analytic value $-0.344166 \cdots[18,19]$. 

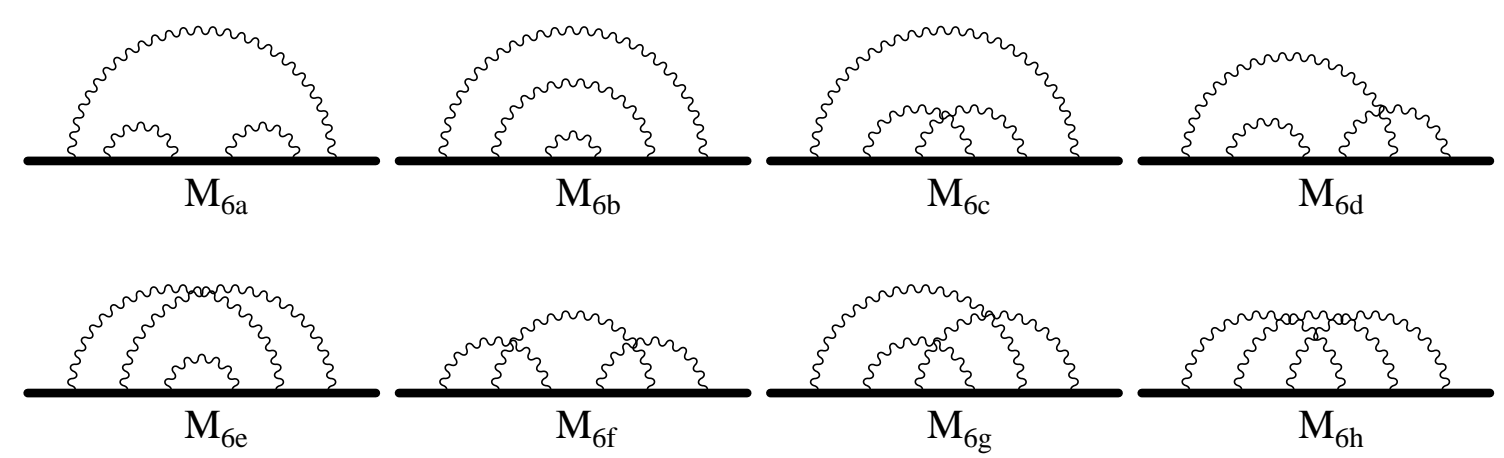

FIG. 5: Sixth-order $q$-type self-energy-like diagrams $M_{6 x}, x=a, \ldots, h$. The time reversal diagrams of $M_{6 d}$ and $M_{6 g}$ are not shown here. The fermion lines of a diagram is named 1 to 5 from left to right. The vertex diagram obtained by inserting an external photon vertex into the fermion line $i$ of the self-energy diagram $6 x$ is named $6 x i$.

\section{Sixth-order $a_{e}$}

The sixth-order diagrams can be evaluated in a similar manner and are found to give a result in good agreement with the numerically [20] and analytically [21] known values as follows. Fifty vertex diagrams of the sixth-order $q$-type diagrams are reduced to eight self-energy-like diagrams shown in Fig. 5 by means of the Ward-Takahashi identity and the time-reversal symmetry. It takes just one minute to create all eight FORTRAN programs for $M_{6 x}(x=a, b, \ldots, h)$ by GENCODE $N$ on $h p$ 's Alpha machine. Numerical evaluation was carried out on RIKEN's PC-cluster system (RSCC). After computation of 2 to 6 wall-clock hours with 16 Xeon-CPU's for each diagram to carry out a VEGAS integration with one hundred million sampling points per iteration for 450 iterations, we obtained

$$
\Delta M^{(6) \text { new }}=\sum_{x=a}^{h} \Delta M_{6 x}^{\text {new }}=0.4260(11) .
$$

After continuing computation with one billion sampling points per iteration for 200 iterations for each diagram, we obtain the updated result $\Delta M^{(6) \text { new }}$ given in Eq. (80). The contribution of $q$-type diagrams to $A_{1}^{(6)}$ including the residual renormalization is given by

$$
\begin{aligned}
A_{1}^{(6)}(q \text {-type }) & =\Delta M^{(6) \text { new }}-3 \Delta M^{(4)} \Delta B_{2}+M_{2}\left\{-\Delta B^{(4)}-\Delta L^{(4)}+2\left(\Delta B_{2}\right)^{2}\right\} \\
& =0.90526(53),
\end{aligned}
$$

where $\Delta B(L)^{(4)}$ is the sum of the finite parts of the fourth-order wave function (vertex) renormalization constants. See Appendix C2 for their definitions. The formula of the 
residual renormalization (A5) can be obtained by using the definitions of the finite quantities $\Delta M_{4 a(b)}, \Delta M_{6 x}$, etc., in Appendixes $\mathrm{B}$ and $\mathrm{C}$, The values of finite quantities such as $\Delta L_{4 c} \ldots$ are given in Table $\mathrm{V}$. The various finite pieces appearing in (A5) are

$$
\begin{aligned}
& \Delta B^{(4)}=\Delta B_{4 a}+\Delta B_{4 b}=-0.437094(21) \\
& \Delta L^{(4)}=2 \Delta L_{4 c}+\Delta L_{4 x}+2 \Delta L_{4 s}+\Delta L_{4 l}=0.465024(17) \\
& \Delta M^{(4)}=\Delta M_{4 a}+\Delta M_{4 b}=0.030804 \cdots \quad \text { (known exactly ). }
\end{aligned}
$$

Eq. (A5) again shows good agreement with the analytic result $0.904979 \cdots$ by Laporta and Remiddi [21].

\section{APPENDIX B: DIVERGENCE STRUCTURE OF THE MAGNETIC MOMENTS}

We briefly summarize our notation in Appendices $\mathrm{B}$ and $\mathrm{C}$. The relation between the unrenormalized amplitude $M$ and the finite amplitude $\Delta M$ of magnetic moment is listed in this appendix.

A symbol with a prefix $\Delta$ means a finite quantity. A renormalization constant with a superscript "UV", $A^{\mathrm{UV}}$, is the leading UV-divergent term of the on-shell renormalization constant $A$. $A$ can be $L, B$, and $\delta m$ according to a vertex-, wave-function, and massrenormalization constant, respectively. $A^{\mathrm{UV}}$ is identical with $\hat{A}$ in Refs. [16, 36]. The subtraction terms proportional to a UV-renormalization term $A^{\mathrm{UV}}$ are generated by the $K$-operations. A renormalization constant with a superscript " $\mathrm{R}$ ", such as $L_{4 s}^{\mathrm{R}}$, is the residual term defined in Eqs. (27) and (29). $A$ of $A^{\mathrm{R}}$ must be either $L$ or $\delta m$. $B^{\mathrm{R}}$ is also defined accordingly, but there appears no $B^{\mathrm{R}}$ term in the definition of a finite magnetic moment amplitude. The subtraction terms involving $A^{\mathrm{R}}$ are generated by the $R / I$-subtraction operations.

The subscript of $M$ or $A$ stands for the name of a diagram. A self-energy-like diagram of the second-, fourth-, and sixth-orders are called $2,4 a$ and $4 b$, and $6 a, 6 b, 6 c, 6 d, 6 e, 6 f, 6 g$, and $6 h$, respectively. (See Figs. 4 and 5.) The 47 independent self-energy-like diagrams of the eighth-order are named from 01 to 47. (See Fig. 1.) The fermion lines are always named from 1 to $n-1$ from the left to right, where $n$ is the order of the perturbation theory.

The name of a vertex diagram is determined based on the Ward-Takahashi related selfenergy diagram. When the vertex diagram is obtained by inserting the external photon 
into the fermion line $i$ of a self-energy diagram $n x$, this is called $n x i$. Thus, we have $L_{4 x i}, i=1,2,3, x=a, b$, and $L_{6 x i}, i=1, \cdots 5, x=a, \cdots h$ for the fourth-, and sixth-order vertex renormalization constants, respectively. The vertex renormalization constant of the second-order is named $L_{2}$, since there is only one vertex diagam of the second order. In the early article [40], the fourth-order vertex diagrams are given other names. We follow the naming system Ref.[40] in this paper. The correspondence between two names of the fourth-order vertex diagrams is that $4 a 1=4 a 3=4 c, 4 a 2=4 x, 4 b 1=4 b 3=4 s$, and $4 b 2=4 l$. (See Fig. 4).

When a two-point vertex is inserted into the fermion line $i$ of a diagram $n x$, the resulting diagram is called $n x\left(i^{\star}\right)$. Namely, $\star$ indicates the two-point vertex.

The primed quantity, for example $L_{4 x\left(i^{\prime}\right)}$, is the derivative amplitude obtained by applying

$-z_{i} \frac{\partial}{\partial z_{i}}$ operation on the integrand, where $z_{i}$ is the Feynman parameter assigned to the fermion line $i$. Note that $L_{4 x\left(i^{\prime}\right)}$ is equal to $L_{4 x}$, but its $U V$-divergent part $L_{4 x\left(i^{\prime}\right)}^{\mathrm{UV}}$ is not equal to $L_{4 x}^{\mathrm{UV}}$. Since second order quantities such as $B_{2}$ and $\delta m_{2}$ have only one electron line, it is not really necessary to distinguish different electron lines. We therefore use somewhat sloppy notations $B_{2^{\star}}$ and $B_{2^{\prime}}$ instead of $B_{2\left(1^{\star}\right)}$ and $B_{2\left(1^{\prime}\right)}$.

For $L_{2}$, which contains electron lines 1 and 2 , it is sometimes necessary to distinguish lines in which insertion is made. $L_{2^{\star \star \dagger}}$ implies that two two-point vertices are inserted into the fermion line 1 of $L_{2}$, while $L_{2^{\star \dagger \star}}$ means that one two-point vertex is inserted into the line 1 and another into the line 2. $M_{4 a}$ contains three electron lines $1,2,3$ and $M_{4 a\left(1^{\star \star}\right)}$ means that two-point vertex insertion has been made twice in the electron line 1 , and so on.

\section{Fourth-order magnetic moments}

The fourth-order magnetic moments are the same for both old and new approaches. The UV-finite amplitude are also given here.

$$
\begin{aligned}
& M_{4 a}=\Delta M_{4 a}+2 L_{2}^{\mathrm{UV}} M_{2} \\
& \underline{M}_{4 a}=\Delta M_{4 a} \\
& M_{4 b}=\Delta M_{4 b}+B_{2}^{\mathrm{UV}} M_{2}+\delta m_{2} M_{2^{\star}}+L_{2}^{\mathrm{R}} M_{2} \\
& \underline{M}_{4 b}=\Delta M_{4 b}+L_{2}^{\mathrm{R}} M_{2}
\end{aligned}
$$

In the new approach no explicit form of $M_{4^{\star}}$ is needed because the mass renormalization 
is completed by the $R$-subtraction operation. They are, however, listed here, since they are used in the old approach.

$$
\begin{aligned}
& M_{4 a\left(1^{\star}\right)}=\Delta M_{4 a\left(1^{\star}\right)}+L_{2}^{\mathrm{UV}} M_{2^{\star}}+I_{4 a\left(1^{\star}\right)} \\
& M_{4 a\left(2^{\star}\right)}=\Delta M_{4 a\left(2^{\star}\right)}+I_{4 a\left(2^{\star}\right)} \\
& M_{4 b\left(1^{\star}\right)}=\Delta M_{4 b\left(1^{\star}\right)}+\left(\delta m_{2} M_{2^{\star \star}}+B_{2}^{\mathrm{UV}} M_{2^{\star}}\right)+I_{4 b\left(1^{\star}\right)} \\
& M_{4 b\left(2^{\star}\right)}=\Delta M_{4 b\left(2^{\star}\right)}+\delta m_{2^{\star}}^{\mathrm{UV}} M_{2^{\star}}+I_{4 b\left(2^{\star}\right)}+L_{2}^{\mathrm{R}} M_{2^{\star}}+M_{2^{\star}}[I] \delta \widetilde{m}_{2^{\star}}-2 M_{2^{\star}}[I] L_{2}^{\mathrm{R}}
\end{aligned}
$$

\section{Sixth-order magnetic moments by GENCODEN}

The finite amplitudes of the sixth-order are given in the following. For simplicity, we drop the superscript "new" from $\Delta M_{6 x}^{\text {new }}$.

$$
\begin{aligned}
M_{6 a} & =\Delta M_{6 a}+2 \delta m_{2} M_{4 b\left(1^{\star}\right)}+2 B_{2}^{\mathrm{UV}} M_{4 b}-\delta m_{2}\left(\delta m_{2} M_{2^{\star \star}}+B_{2}^{\mathrm{UV}} M_{2^{\star}}\right) \\
& -B_{2}^{\mathrm{UV}}\left(\delta m_{2} M_{2^{\star}}+B_{2}^{\mathrm{UV}} M_{2}\right) \\
& +2 L_{4 s}^{\mathrm{R}} M_{2} \\
M_{6 b} & =\Delta M_{6 b}+\delta m_{2} M_{4 b\left(2^{\star}\right)}+B_{2}^{\mathrm{UV}} M_{4 b}+\delta m_{4 b}^{\mathrm{UV}} M_{2^{\star}}+B_{4 b}^{\mathrm{UV}} M_{2}-\delta m_{2} \delta m_{2^{\star}}^{\mathrm{UV}} M_{2^{\star}} \\
& -B_{2}^{\mathrm{UV}}\left(\delta m_{2^{\prime}}^{\mathrm{UV}} M_{2^{\star}}+B_{2^{\prime}}^{\mathrm{UV}} M_{2}\right) \\
& +L_{2}^{\mathrm{R}} \Delta M_{4 b}+L_{4 l}^{\mathrm{R}} M_{2}+M_{2^{\star}} \Delta \delta m_{4 b} \\
M_{6 c} & =\Delta M_{6 c}+2 L_{2}^{\mathrm{UV}} M_{4 b}+\delta m_{4 a}^{\mathrm{UV}} M_{2^{\star}}+B_{4 a}^{\mathrm{UV}} M_{2}-2 L_{2}^{\mathrm{UV}}\left(\delta m_{2} M_{2^{\star}}+B_{2}^{\mathrm{UV}} M_{2}\right) \\
& +L_{2}^{\mathrm{R}} \Delta M_{4 a}+M_{2^{\star}} \Delta \delta m_{4 a} \\
M_{6 d} & =\Delta M_{6 d}+L_{4 s}^{\mathrm{UV}} M_{2}+\delta m_{2} M_{4 a\left(1^{\star}\right)}+B_{2}^{\mathrm{UV}} M_{4 a}+L_{2}^{\mathrm{UV}} M_{4 b}-B_{2}^{\mathrm{UV}} L_{2^{\prime}}^{\mathrm{UV}} M_{2} \\
& -L_{2}^{\mathrm{UV}}\left(\delta m_{2} M_{2^{\star}}+B_{2}^{\mathrm{UV}} M_{2}\right) \\
& +L_{4 c}^{\mathrm{R}} M_{2} \\
M_{6 e} & =\Delta M_{6 e}+2 L_{4 s}^{\mathrm{UV}} M_{2}+\delta m_{2} M_{4 a\left(2^{\star}\right)}+B_{2}^{\mathrm{UV}} M_{4 a}-2 L_{2^{\prime}}^{\mathrm{UV}} B_{2}^{\mathrm{UV}} M_{2} \\
& +L_{4 x}^{\mathrm{R}} M_{2} \\
M_{6 f} & =\Delta M_{6 f}+2 L_{4 c}^{\mathrm{UV}} M_{2}+2 L_{2}^{\mathrm{UV}} M_{4 a}-3 L_{2}^{\mathrm{UV}} L_{2}^{\mathrm{UV}} M_{2} \\
M_{6 g} & =\Delta M_{6 g}+L_{4 c}^{\mathrm{UV}} M_{2}+L_{4 l}^{\mathrm{UV}} M_{2}+L_{2}^{\mathrm{UV}} M_{4 a}-2 L_{2}^{\mathrm{UV}} L_{2}^{\mathrm{UV}} M_{2} \\
M_{6 h}+2 L_{4 x}^{\mathrm{UV}} M_{2} & \Delta M_{6 h} \\
& =\Delta
\end{aligned}
$$




\section{3. eighth-order magnetic moments by GENCODE $N$}

The finite amplitudes of the eighth-order are given in the following. For simplicity, we drop the superscript "new" from $\Delta M_{i}^{\text {new }}$.

$$
\begin{aligned}
& M_{01}=\Delta M_{01}+2 L_{2}^{\mathrm{UV}} M_{6 f}+2 L_{4 c}^{\mathrm{UV}} M_{4 a}+2 L_{6 f 1}^{\mathrm{UV}} M_{2}-3\left(L_{2}^{\mathrm{UV}}\right)^{2} M_{4 a} \\
& -6 L_{2}^{\mathrm{UV}} L_{4 c}^{\mathrm{UV}} M_{2}+4\left(L_{2}^{\mathrm{UV}}\right)^{3} M_{2} \\
& M_{02}=\Delta M_{02}+\delta m_{2} M_{6 f\left(1^{\star}\right)}+B_{2}^{\mathrm{UV}} M_{6 f}+L_{2}^{\mathrm{UV}} M_{6 d}+L_{4 s}^{\mathrm{UV}} M_{4 a}+L_{4 c}^{\mathrm{UV}} M_{4 b}+L_{6 d 5}^{\mathrm{UV}} M_{2} \\
& -L_{2}^{\mathrm{UV}}\left(\delta m_{2} M_{4 a\left(1^{\star}\right)}+B_{2}^{\mathrm{UV}} M_{4 a}\right)-B_{2}^{\mathrm{UV}} L_{2^{\prime}}^{\mathrm{UV}} M_{4 a}-L_{4 c}^{\mathrm{UV}}\left(\delta m_{2} M_{2^{\star}}+B_{2}^{\mathrm{UV}} M_{2}\right) \\
& -B_{2}^{\mathrm{UV}} L_{4 c\left(3^{\prime}\right)}^{\mathrm{UV}} M_{2}-2 L_{2}^{\mathrm{UV}} L_{4 s}^{\mathrm{UV}} M_{2}-\left(L_{2}^{\mathrm{UV}}\right)^{2} M_{4 b}+2 L_{2}^{\mathrm{UV}} B_{2}^{\mathrm{UV}} L_{2^{\prime}}^{\mathrm{UV}} M_{2} \\
& +\left(L_{2}^{\mathrm{UV}}\right)^{2}\left(\delta m_{2} M_{2^{\star}}+B_{2}^{\mathrm{UV}} M_{2}\right) \\
& +L_{6 f 1}^{\mathrm{R}} M_{2} \\
& M_{03}=\Delta M_{03}+2 L_{2}^{\mathrm{UV}} M_{6 d}+\delta m_{2} M_{6 f\left(3^{\star}\right)}+B_{2}^{\mathrm{UV}} M_{6 f}+2 L_{6 d 1}^{\mathrm{UV}} M_{2} \\
& -2 L_{2}^{\mathrm{UV}}\left(\delta m_{2} M_{4 a\left(1^{\star}\right)}+B_{2}^{\mathrm{UV}} M_{4 a}\right)-\left(L_{2}^{\mathrm{UV}}\right)^{2} M_{4 b}-2 L_{2}^{\mathrm{UV}} L_{4 s}^{\mathrm{UV}} M_{2} \\
& -2 B_{2}^{\mathrm{UV}} L_{4 c\left(1^{\prime \prime}\right)}^{\mathrm{UV}} M_{2}+\left(L_{2}^{\mathrm{UV}}\right)^{2}\left(\delta m_{2} M_{2^{\star}}+B_{2}^{\mathrm{UV}} M_{2}\right)+2 L_{2}^{\mathrm{UV}} B_{2}^{\mathrm{UV}} L_{2^{\prime}}^{\mathrm{UV}} M_{2} \\
& +L_{6 f 3}^{\mathrm{R}} M_{2} \\
& \begin{aligned}
M_{04} & =\Delta M_{04}+\delta m_{2} M_{6 d\left(3^{\star}\right)}+B_{2}^{\mathrm{UV}} M_{6 d}+\delta m_{2} M_{6 d\left(1^{\star}\right)}+B_{2}^{\mathrm{UV}} M_{6 d}+L_{2}^{\mathrm{UV}} M_{6 a}+L_{6 a 1}^{\mathrm{UV}} M_{2} \\
& -\delta m_{2}\left(\delta m_{2} M_{4 a\left(1^{\star \star}\right)}+B_{2}^{\mathrm{UV}} M_{4 a\left(1^{\star}\right)}\right)-B_{2}^{\mathrm{UV}}\left(\delta m_{2} M_{4 a\left(1^{\star}\right)}+B_{2}^{\mathrm{UV}} M_{4 a}\right) \\
& -2 L_{2}^{\mathrm{UV}}\left(\delta m_{2} M_{4 b\left(1^{\star}\right)}+B_{2}^{\mathrm{UV}} M_{4 b}\right)-B_{2}^{\mathrm{UV}}\left(L_{4 s\left(3^{\prime}\right)}^{\mathrm{UV}}+L_{4 s\left(1^{\prime \prime}\right)}^{\mathrm{UV}}\right) M_{2} \\
& +L_{2}^{\mathrm{UV}} \delta m_{2}\left(\delta m_{2} M_{2^{\star \star}}+B_{2}^{\mathrm{UV}} M_{2^{\star}}\right)+L_{2}^{\mathrm{UV}} B_{2}^{\mathrm{UV}}\left(\delta m_{2} M_{2^{\star}}+B_{2}^{\mathrm{UV}} M_{2}\right) \\
& +\left(B_{2}^{\mathrm{UV}}\right)^{2} L_{2^{\prime \prime}}^{\mathrm{UV}} M_{2} \\
& +L_{6 d 3}^{\mathrm{R}} M_{2}+L_{6 d 1}^{\mathrm{R}} M_{2}
\end{aligned} \\
& M_{05}=\Delta M_{05}+L_{2}^{\mathrm{UV}} M_{6 h}+L_{4 x}^{\mathrm{UV}} M_{4 a}+L_{6 f 2}^{\mathrm{UV}} M_{2}+L_{6 h 1}^{\mathrm{UV}} M_{2}-3 L_{2}^{\mathrm{UV}} L_{4 x}^{\mathrm{UV}} M_{2} \\
& M_{06}=\Delta M_{06}+L_{2}^{\mathrm{UV}} M_{6 g}+L_{2}^{\mathrm{UV}} M_{6 f}+L_{4 l}^{\mathrm{UV}} M_{4 a}+L_{6 f 3}^{\mathrm{UV}} M_{2}+L_{6 g 5}^{\mathrm{UV}} M_{2} \\
& -2\left(L_{2}^{\mathrm{UV}}\right)^{2} M_{4 a}-2 L_{2}^{\mathrm{UV}} L_{4 l}^{\mathrm{UV}} M_{2}-3 L_{2}^{\mathrm{UV}} L_{4 c}^{\mathrm{UV}} M_{2}+3\left(L_{2}^{\mathrm{UV}}\right)^{3} M_{2}
\end{aligned}
$$




$$
\begin{aligned}
& M_{07}=\Delta M_{07}+L_{2}^{\mathrm{UV}} M_{6 g}+L_{2}^{\mathrm{UV}} M_{6 f}+L_{4 c}^{\mathrm{UV}} M_{4 a}+L_{6 d 2}^{\mathrm{UV}} M_{2}+L_{6 g 1}^{\mathrm{UV}} M_{2} \\
& -2\left(L_{2}^{\mathrm{UV}}\right)^{2} M_{4 a}-4 L_{2}^{\mathrm{UV}} L_{4 c}^{\mathrm{UV}} M_{2}-L_{2}^{\mathrm{UV}} L_{4 l}^{\mathrm{UV}} M_{2}+3\left(L_{2}^{\mathrm{UV}}\right)^{3} M_{2} \\
& M_{08}=\Delta M_{08}+L_{2}^{\mathrm{UV}} M_{6 c}+2 L_{2}^{\mathrm{UV}} M_{6 d}+\delta m_{4 a}^{\mathrm{UV}} M_{4 a\left(1^{\star}\right)}+B_{4 a}^{\mathrm{UV}} M_{4 a}+L_{6 c 1}^{\mathrm{UV}} M_{2} \\
& -2\left(L_{2}^{\mathrm{UV}}\right)^{2} M_{4 b}-L_{2}^{\mathrm{UV}}\left(\delta m_{4 a}^{\mathrm{UV}} M_{2^{\star}}+B_{4 a}^{\mathrm{UV}} M_{2}\right)-2 L_{2}^{\mathrm{UV}}\left(\delta m_{2} M_{4 a\left(1^{\star}\right)}+B_{2}^{\mathrm{UV}} M_{4 a}\right) \\
& -2 L_{2}^{\mathrm{UV}} L_{4 s}^{\mathrm{UV}} M_{2}-B_{4 a}^{\mathrm{UV}} L_{2^{\prime}}^{\mathrm{UV}} M_{2}+2\left(L_{2}^{\mathrm{UV}}\right)^{2}\left(\delta m_{2} M_{2^{\star}}+B_{2}^{\mathrm{UV}} M_{2}\right) \\
& +2 L_{2}^{\mathrm{UV}} B_{2}^{\mathrm{UV}} L_{2^{\prime}}^{\mathrm{UV}} M_{2} \\
& +L_{4 c}^{\mathrm{R}} \Delta M_{4 a}+\underline{M}_{4 a\left(1^{\star}\right)} \Delta \delta m_{4 a} \\
& M_{09}=\Delta M_{09}+\delta m_{2} M_{6 f\left(2^{\star}\right)}+B_{2}^{\mathrm{UV}} M_{6 f}+L_{2}^{\mathrm{UV}} M_{6 e}+L_{4 s}^{\mathrm{UV}} M_{4 a}+L_{6 e 1}^{\mathrm{UV}} M_{2}+L_{6 d 3}^{\mathrm{UV}} M_{2} \\
& -L_{2}^{\mathrm{UV}}\left(\delta m_{2} M_{4 a\left(2^{\star \star}\right)}+B_{2}^{\mathrm{UV}} M_{4 a}\right)-B_{2}^{\mathrm{UV}} L_{2^{\prime}}^{\mathrm{UV}} M_{4 a}-B_{2}^{\mathrm{UV}} L_{4 c\left(2^{\prime}\right)}^{\mathrm{UV}} M_{2} \\
& -B_{2}^{\mathrm{UV}} L_{4 c\left(1^{\prime}\right)}^{\mathrm{UV}} M_{2}-3 L_{2}^{\mathrm{UV}} L_{4 s}^{\mathrm{UV}} M_{2}+3 L_{2}^{\mathrm{UV}} B_{2}^{\mathrm{UV}} L_{2^{\prime}}^{\mathrm{UV}} M_{2} \\
& +L_{6 f 2}^{\mathrm{R}} M_{2} \\
& M_{10}=\Delta M_{10}+\delta m_{2} M_{6 d\left(2^{\star}\right)}+B_{2}^{\mathrm{UV}} M_{6 d}+\delta m_{4 b}^{\mathrm{UV}} M_{4 a\left(1^{\star}\right)}+B_{4 b}^{\mathrm{UV}} M_{4 a} \\
& +L_{2}^{\mathrm{UV}} M_{6 b}+L_{6 b 1}^{\mathrm{UV}} M_{2}-\delta m_{2} \delta m_{2^{\star}}^{\mathrm{UV}} M_{4 a\left(1^{\star}\right)}-B_{2}^{\mathrm{UV}}\left(\delta m_{2^{\prime}}^{\mathrm{UV}} M_{4 a\left(1^{\star}\right)}+B_{2^{\prime}}^{\mathrm{UV}} M_{4 a}\right) \\
& -L_{2}^{\mathrm{UV}}\left(\delta m_{2} M_{4 b\left(2^{\star}\right)}+B_{2}^{\mathrm{UV}} M_{4 b}\right)-B_{2}^{\mathrm{UV}} L_{4 s\left(2^{\prime}\right)}^{\mathrm{UV}} M_{2} \\
& -L_{2}^{\mathrm{UV}}\left(\delta m_{4 b}^{\mathrm{UV}} M_{2^{\star}}+B_{4 b}^{\mathrm{UV}} M_{2}\right)-B_{4 b}^{\mathrm{UV}} L_{2^{\prime}}^{\mathrm{UV}} M_{2}+L_{2}^{\mathrm{UV}} \delta m_{2} \delta m_{2^{\star}}^{\mathrm{UV}} M_{2^{\star}} \\
& +L_{2}^{\mathrm{UV}} B_{2}^{\mathrm{UV}}\left(\delta m_{2^{\prime}}^{\mathrm{UV}} M_{2^{\star}}+B_{2^{\prime}}^{\mathrm{UV}} M_{2}\right)+B_{2}^{\mathrm{UV}} B_{2^{\prime}}^{\mathrm{UV}} L_{2^{\prime}}^{\mathrm{UV}} M_{2} \\
& +L_{4 c}^{\mathrm{R}}\left(\Delta M_{4 b}+L_{2}^{\mathrm{R}} M_{2}\right)-L_{4 c}^{\mathrm{R}} L_{2}^{\mathrm{R}} M_{2}+L_{6 d 2}^{\mathrm{R}} M_{2}+\underline{M}_{4 a\left(1^{\star}\right)} \Delta \delta m_{4 b} \\
& M_{11}=\Delta M_{11}+2 \delta m_{2} M_{6 d\left(5^{\star}\right)}+2 B_{2}^{\mathrm{UV}} M_{6 d}+2 L_{4 s}^{\mathrm{UV}} M_{4 b} \\
& -\delta m_{2}\left(\delta m_{2} M_{4 a\left(1^{\star} 3^{\star}\right)}+B_{2}^{\mathrm{UV}} M_{4 a\left(1^{\star}\right)}\right)-B_{2}^{\mathrm{UV}}\left(\delta m_{2} M_{4 a\left(1^{\star}\right)}+B_{2}^{\mathrm{UV}} M_{4 a}\right) \\
& -2 L_{4 s}^{\mathrm{UV}}\left(\delta m_{2} M_{2^{\star}}+B_{2}^{\mathrm{UV}} M_{2}\right)-2 B_{2}^{\mathrm{UV}} L_{2^{\prime}}^{\mathrm{UV}} M_{4 b} \\
& +2 B_{2}^{\mathrm{UV}} L_{2^{\prime}}^{\mathrm{UV}}\left(\delta m_{2} M_{2^{\star}}+B_{2}^{\mathrm{UV}} M_{2}\right) \\
& +2 L_{6 d 5}^{\mathrm{R}} M_{2}
\end{aligned}
$$




$$
\begin{aligned}
M_{12} & =\Delta M_{12}+2 \delta m_{2} M_{6 a\left(1^{\star}\right)}+2 B_{2}^{\mathrm{UV}} M_{6 a}+\delta m_{2} M_{6 a\left(3^{\star}\right)}+B_{2}^{\mathrm{UV}} M_{6 a} \\
& -2 \delta m_{2}\left(\delta m_{2} M_{4 b\left(1^{\star \star}\right)}+B_{2}^{\mathrm{UV}} M_{4 b\left(1^{\star}\right)}\right)-2 B_{2}^{\mathrm{UV}}\left(\delta m_{2} M_{4 b\left(1^{\star}\right)}+B_{2}^{\mathrm{UV}} M_{4 b}\right) \\
& -\delta m_{2}\left(\delta m_{2} M_{4 b\left(1^{\star} 3^{\star}\right)}+B_{2}^{\mathrm{UV}} M_{4 b\left(1^{\star}\right)}\right)-B_{2}^{\mathrm{UV}}\left(\delta m_{2} M_{4 b\left(1^{\star}\right)}+B_{2}^{\mathrm{UV}} M_{4 b}\right) \\
& +\left(\delta m_{2}\right)^{2}\left(\delta m_{2} M_{2^{\star \star \star}}+B_{2}^{\mathrm{UV}} M_{2^{\star \star}}\right)+2 \delta m_{2} B_{2}^{\mathrm{UV}}\left(\delta m_{2} M_{2^{\star \star}}+B_{2}^{\mathrm{UV}} M_{2^{\star}}\right) \\
& +\left(B_{2}^{\mathrm{UV}}\right)^{2}\left(\delta m_{2} M_{2^{\star}}+B_{2}^{\mathrm{UV}} M_{2}\right) \\
& +2 L_{6 a 1}^{\mathrm{R}} M_{2}+L_{6 a 3}^{\mathrm{R}} M_{2}
\end{aligned}
$$

$$
\begin{aligned}
M_{13} & =\Delta M_{13}+\delta m_{2} M_{6 h\left(1^{\star}\right)}+B_{2}^{\mathrm{UV}} M_{6 h}+L_{4 x}^{\mathrm{UV}} M_{4 b}+L_{6 d 4}^{\mathrm{UV}} M_{2} \\
& -L_{4 x}^{\mathrm{UV}}\left(\delta m_{2} M_{2^{\star}}+B_{2}^{\mathrm{UV}} M_{2}\right)-B_{2}^{\mathrm{UV}} L_{4 x\left(1^{\prime}\right)}^{\mathrm{UV}} M_{2} \\
& +L_{6 h 1}^{\mathrm{R}} M_{2}
\end{aligned}
$$

$$
\begin{aligned}
M_{14} & =\Delta M_{14}+\delta m_{2} M_{6 g\left(5^{\star}\right)}+B_{2}^{\mathrm{UV}} M_{6 g}+L_{2}^{\mathrm{UV}} M_{6 d}+L_{4 l}^{\mathrm{UV}} M_{4 b}+L_{6 d 3}^{\mathrm{UV}} M_{2} \\
& -L_{2}^{\mathrm{UV}}\left(\delta m_{2} M_{4 a\left(1^{\star}\right)}+B_{2}^{\mathrm{UV}} M_{4 a}\right)-L_{4 l}^{\mathrm{UV}}\left(\delta m_{2} M_{2^{\star}}+B_{2}^{\mathrm{UV}} M_{2}\right)-B_{2}^{\mathrm{UV}} L_{4 c\left(1^{\prime}\right)}^{\mathrm{UV}} M_{2} \\
& -\left(L_{2}^{\mathrm{UV}}\right)^{2} M_{4 b}-L_{2}^{\mathrm{UV}} L_{4 s}^{\mathrm{UV}} M_{2}+\left(L_{2}^{\mathrm{UV}}\right)^{2}\left(\delta m_{2} M_{2^{\star}}+B_{2}^{\mathrm{UV}} M_{2}\right)+L_{2}^{\mathrm{UV}} B_{2}^{\mathrm{UV}} L_{2^{\prime}}^{\mathrm{UV}} M_{2} \\
& +L_{6 g 5}^{\mathrm{R}} M_{2}
\end{aligned}
$$

$$
\begin{aligned}
M_{15} & =\Delta M_{15}+\delta m_{2} M_{6 g\left(1^{\star}\right)}+B_{2}^{\mathrm{UV}} M_{6 g}+L_{2}^{\mathrm{UV}} M_{6 d}+L_{4 c}^{\mathrm{UV}} M_{4 b}+L_{6 a 2}^{\mathrm{UV}} M_{2} \\
& -L_{2}^{\mathrm{UV}}\left(\delta m_{2} M_{4 a\left(1^{\star}\right)}+B_{2}^{\mathrm{UV}} M_{4 a}\right)-L_{4 c}^{\mathrm{UV}}\left(\delta m_{2} M_{2^{\star}}+B_{2}^{\mathrm{UV}} M_{2}\right)-B_{2}^{\mathrm{UV}} L_{4 l\left(1^{\prime}\right)}^{\mathrm{UV}} M_{2} \\
& -\left(L_{2}^{\mathrm{UV}}\right)^{2} M_{4 b}-L_{2}^{\mathrm{UV}} L_{4 s}^{\mathrm{UV}} M_{2} \\
& +\left(L_{2}^{\mathrm{UV}}\right)^{2}\left(\delta m_{2} M_{2^{\star}}+B_{2}^{\mathrm{UV}} M_{2}\right)+L_{2}^{\mathrm{UV}} B_{2}^{\mathrm{UV}} L_{2^{\prime}}^{\mathrm{UV}} M_{2} \\
& +L_{6 g 1}^{\mathrm{R}} M_{2}
\end{aligned}
$$

$$
\begin{aligned}
M_{16} & =\Delta M_{16}+\delta m_{2} M_{6 c\left(1^{\star}\right)}+B_{2}^{\mathrm{UV}} M_{6 c}+\delta m_{4 a}^{\mathrm{UV}} M_{4 b\left(1^{\star}\right)}+B_{4 a}^{\mathrm{UV}} M_{4 b}+2 L_{2}^{\mathrm{UV}} M_{6 a} \\
& -\delta m_{2}\left(\delta m_{4 a}^{\mathrm{UV}} M_{2^{\star \star}}+B_{4 a}^{\mathrm{UV}} M_{2^{\star}}\right)-B_{2}^{\mathrm{UV}}\left(\delta m_{4 a}^{\mathrm{UV}} M_{2^{\star}}+B_{4 a}^{\mathrm{UV}} M_{2}\right) \\
& -4 L_{2}^{\mathrm{UV}}\left(\delta m_{2} M_{4 b\left(1^{\star}\right)}+B_{2}^{\mathrm{UV}} M_{4 b}\right) \\
& +2 L_{2}^{\mathrm{UV}} \delta m_{2}\left(\delta m_{2} M_{2^{\star \star}}+B_{2}^{\mathrm{UV}} M_{2^{\star}}\right)+2 B_{2}^{\mathrm{UV}} L_{2}^{\mathrm{UV}}\left(\delta m_{2} M_{2^{\star}}+B_{2}^{\mathrm{UV}} M_{2}\right) \\
& +L_{6 c 1}^{\mathrm{R}} M_{2}+L_{4 s}^{\mathrm{R}} \Delta M_{4 a}+M_{4 b\left(1^{\star}\right.} \Delta \delta m_{4 a}-L_{2^{\star}}^{\mathrm{R}} \Delta \delta m_{4 a} M_{2}
\end{aligned}
$$




$$
\begin{aligned}
& M_{17}=\Delta M_{17}+\delta m_{2} M_{6 e\left(1^{\star}\right)}+B_{2}^{\mathrm{UV}} M_{6 e}+\delta m_{2} M_{6 d\left(4^{\star}\right)}+B_{2}^{\mathrm{UV}} M_{6 d}+L_{4 s}^{\mathrm{UV}} M_{4 b} \\
& +L_{6 a 3}^{\mathrm{UV}} M_{2}-\delta m_{2}\left(\delta m_{2} M_{4 a\left(1^{\star} 2^{\star}\right)}+B_{2}^{\mathrm{UV}} M_{4 a\left(1^{\star}\right)}\right) \\
& -B_{2}^{\mathrm{UV}}\left(\delta m_{2} M_{4 a\left(2^{\star}\right)}+B_{2}^{\mathrm{UV}} M_{4 a}\right)-L_{4 s}^{\mathrm{UV}}\left(\delta m_{2} M_{2^{\star}}+B_{2}^{\mathrm{UV}} M_{2}\right)-2 B_{2}^{\mathrm{UV}} L_{4 s\left(1^{\prime}\right)}^{\mathrm{UV}} M_{2} \\
& -B_{2}^{\mathrm{UV}} L_{2^{\prime}}^{\mathrm{UV}} M_{4 b}+\delta m_{2} B_{2}^{\mathrm{UV}} L_{2^{\prime}}^{\mathrm{UV}} M_{2^{\star}}+\left(B_{2}^{\mathrm{UV}}\right)^{2}\left(L_{2^{\prime}}^{\mathrm{UV}}+L_{2^{\prime \prime}}^{\mathrm{UV}}\right) M_{2} \\
& +L_{6 e 1}^{\mathrm{R}} M_{2}+L_{6 d 4}^{\mathrm{R}} M_{2} \\
& M_{18}=\Delta M_{18}+\delta m_{2} M_{6 b\left(1^{\star}\right)}+B_{2}^{\mathrm{UV}} M_{6 b}+\delta m_{2} M_{6 a\left(2^{\star}\right)}+B_{2}^{\mathrm{UV}} M_{6 a}+\delta m_{4 b}^{\mathrm{UV}} M_{4 b\left(1^{\star}\right)} \\
& +B_{4 b}^{\mathrm{UV}} M_{4 b}-\delta m_{2}\left(\delta m_{2} M_{4 b\left(1^{\star} 2^{\star}\right)}+B_{2}^{\mathrm{UV}} M_{4 b\left(1^{\star}\right)}\right)-B_{2}^{\mathrm{UV}}\left(\delta m_{2} M_{4 b\left(2^{\star}\right)}+B_{2}^{\mathrm{UV}} M_{4 b}\right) \\
& -\delta m_{2}\left(\delta m_{4 b}^{\mathrm{UV}} M_{2^{\star \star}}+B_{4 b}^{\mathrm{UV}} M_{2^{\star}}\right)-B_{2}^{\mathrm{UV}}\left(\delta m_{4 b}^{\mathrm{UV}} M_{2^{\star}}+B_{4 b}^{\mathrm{UV}} M_{2}\right) \\
& -\delta m_{2} \delta m_{2^{\star}}^{\mathrm{UV}} M_{4 b\left(1^{\star}\right)}-B_{2}^{\mathrm{UV}}\left(\delta m_{2^{\prime}}^{\mathrm{UV}} M_{4 b\left(1^{\star}\right)}+B_{2^{\prime}}^{\mathrm{UV}} M_{4 b}\right) \\
& +\delta m_{2} \delta m_{2^{\star}}^{\mathrm{UV}}\left(\delta m_{2} M_{2^{\star \star}}+B_{2}^{\mathrm{UV}} M_{2^{\star}}\right)+B_{2}^{\mathrm{UV}} \delta m_{2^{\prime}}^{\mathrm{UV}}\left(\delta m_{2} M_{2^{\star \star}}+B_{2}^{\mathrm{UV}} M_{2^{\star}}\right) \\
& +B_{2}^{\mathrm{UV}} B_{2^{\prime}}^{\mathrm{UV}}\left(\delta m_{2} M_{2^{\star}}+B_{2}^{\mathrm{UV}} M_{2}\right) \\
& +L_{6 b 1}^{\mathrm{R}} M_{2}+L_{4 s}^{\mathrm{R}}\left(\Delta M_{4 b}+L_{2}^{\mathrm{R}} M_{2}\right)+L_{6 a 2}^{\mathrm{R}} M_{2}-L_{4 s}^{\mathrm{R}} L_{2}^{\mathrm{R}} M_{2} \\
& +\left(\underline{M}_{4 b\left(1^{\star}\right)}-L_{2^{\star}}^{\mathrm{R}} M_{2}\right) \Delta \delta m_{4 b} \\
& M_{19}=\Delta M_{19}+2 L_{6 h 2}^{\mathrm{UV}} M_{2} \\
& M_{20}=\Delta M_{20}+L_{2}^{\mathrm{UV}} M_{6 h}+L_{6 f 2}^{\mathrm{UV}} M_{2}+L_{6 g 4}^{\mathrm{UV}} M_{2}-2 L_{2}^{\mathrm{UV}} L_{4 x}^{\mathrm{UV}} M_{2} \\
& M_{21}=\Delta M_{21}+2 L_{6 g 2}^{\mathrm{UV}} M_{2} \\
& M_{22}=\Delta M_{22}+L_{2}^{\mathrm{UV}} M_{6 g}+L_{4 c}^{\mathrm{UV}} M_{4 a}+L_{6 f 1}^{\mathrm{UV}} M_{2}+L_{6 c 2}^{\mathrm{UV}} M_{2} \\
& -\left(L_{2}^{\mathrm{UV}}\right)^{2} M_{4 a}-3 L_{2}^{\mathrm{UV}} L_{4 c}^{\mathrm{UV}} M_{2}-L_{2}^{\mathrm{UV}} L_{4 l}^{\mathrm{UV}} M_{2}+2\left(L_{2}^{\mathrm{UV}}\right)^{3} M_{2} \\
& M_{23}=\Delta M_{23}+\delta m_{2} M_{6 h\left(2^{\star}\right)}+B_{2}^{\mathrm{UV}} M_{6 h}+L_{6 e 2}^{\mathrm{UV}} M_{2} \\
& +L_{6 d 4}^{\mathrm{UV}} M_{2}-B_{2}^{\mathrm{UV}}\left(L_{4 x\left(1^{\prime}\right)}^{\mathrm{UV}}+L_{4 x\left(2^{\prime}\right)}^{\mathrm{UV}}\right) M_{2} \\
& +L_{6 h 2}^{\mathrm{R}} M_{2} \\
& M_{24}=\Delta M_{24}+\delta m_{2} M_{6 g\left(2^{\star}\right)}+B_{2}^{\mathrm{UV}} M_{6 g}+L_{4 s}^{\mathrm{UV}} M_{4 a}+L_{6 b 2}^{\mathrm{UV}} M_{2} \\
& +L_{6 d 5}^{\mathrm{UV}} M_{2}-B_{2}^{\mathrm{UV}} L_{2^{\prime}}^{\mathrm{UV}} M_{4 a}-B_{2}^{\mathrm{UV}} L_{4 l 2^{\prime}}^{\mathrm{UV}} M_{2} \\
& -2 L_{4 s}^{\mathrm{UV}} L_{2}^{\mathrm{UV}} M_{2}-B_{2}^{\mathrm{UV}} L_{4 c\left(3^{\prime}\right)}^{\mathrm{UV}} M_{2}+2 L_{2}^{\mathrm{UV}} B_{2}^{\mathrm{UV}} L_{2^{\prime}}^{\mathrm{UV}} M_{2} \\
& +L_{6 g 2}^{\mathrm{R}} M_{2}
\end{aligned}
$$




$$
\begin{aligned}
& M_{25}=\Delta M_{25}+2 L_{2}^{\mathrm{UV}} M_{6 g}+2 L_{6 d 2}^{\mathrm{UV}} M_{2}-\left(L_{2}^{\mathrm{UV}}\right)^{2} M_{4 a}-2 L_{2}^{\mathrm{UV}} L_{4 l}^{\mathrm{UV}} M_{2} \\
& -2 L_{2}^{\mathrm{UV}} L_{4 c}^{\mathrm{UV}} M_{2}+2\left(L_{2}^{\mathrm{UV}}\right)^{3} M_{2} \\
& M_{26}=\Delta M_{26}+2 L_{2}^{\mathrm{UV}} M_{6 c}+2 L_{4 c}^{\mathrm{UV}} M_{4 b}+\delta m_{6 f}^{\mathrm{UV}} M_{2^{\star}}+B_{6 f}^{\mathrm{UV}} M_{2}-3\left(L_{2}^{\mathrm{UV}}\right)^{2} M_{4 b} \\
& -2 L_{2}^{\mathrm{UV}}\left(\delta m_{4 a}^{\mathrm{UV}} M_{2^{\star}}+B_{4 a}^{\mathrm{UV}} M_{2}\right)-2 L_{4 c}^{\mathrm{UV}}\left(\delta m_{2} M_{2^{\star}}+B_{2}^{\mathrm{UV}} M_{2}\right) \\
& +3\left(L_{2}^{\mathrm{UV}}\right)^{2}\left(\delta m_{2} M_{2^{\star}}+B_{2}^{\mathrm{UV}} M_{2}\right) \\
& +L_{2}^{\mathrm{R}} \Delta M_{6 f}+M_{2^{\star}} \Delta \delta m_{6 f} \\
& M_{27}=\Delta M_{27}+\delta m_{2} M_{6 g\left(4^{\star}\right)}+B_{2}^{\mathrm{UV}} M_{6 g}+L_{2}^{\mathrm{UV}} M_{6 e}+L_{6 d 1}^{\mathrm{UV}} M_{2}+L_{6 a 2}^{\mathrm{UV}} M_{2} \\
& -B_{2}^{\mathrm{UV}} L_{4 l\left(1^{\prime}\right)}^{\mathrm{UV}} M_{2}-B_{2}^{\mathrm{UV}} L_{4 c\left(1^{\prime \prime}\right)}^{\mathrm{UV}} M_{2}-2 L_{2}^{\mathrm{UV}} L_{4 s}^{\mathrm{UV}} M_{2}-L_{2}^{\mathrm{UV}}\left(\delta m_{2} M_{4 a\left(2^{\star}\right)}\right. \\
& \left.+B_{2}^{\mathrm{UV}} M_{4 a}\right)+2 L_{2}^{\mathrm{UV}} B_{2}^{\mathrm{UV}} L_{2^{\prime}}^{\mathrm{UV}} M_{2} \\
& +L_{6 g 4}^{\mathrm{R}} M_{2} \\
& M_{28}=\Delta M_{28}+\delta m_{2} M_{6 c\left(2^{\star}\right)}+B_{2}^{\mathrm{UV}} M_{6 c}+L_{2}^{\mathrm{UV}} M_{6 b}+L_{4 s}^{\mathrm{UV}} M_{4 b}+\delta m_{6 d}^{\mathrm{UV}} M_{2^{\star}}+B_{6 d}^{\mathrm{UV}} M_{2} \\
& -L_{2}^{\mathrm{UV}}\left(\delta m_{2} M_{4 b\left(2^{\star}\right)}+B_{2}^{\mathrm{UV}} M_{4 b}\right)-B_{2}^{\mathrm{UV}} L_{2^{\prime}}^{\mathrm{UV}} M_{4 b}-\delta m_{2} \delta m_{4 a\left(1^{\star}\right)}^{\mathrm{UV}} M_{2^{\star}} \\
& -B_{2}^{\mathrm{UV}}\left(\delta m_{4 a\left(1^{\prime}\right)}^{\mathrm{UV}} M_{2^{\star}}+B_{4 a\left(1^{\prime}\right)}^{\mathrm{UV}} M_{2}\right)-L_{2}^{\mathrm{UV}}\left(\delta m_{4 b}^{\mathrm{UV}} M_{2^{\star}}+B_{4 b}^{\mathrm{UV}} M_{2}\right) \\
& -L_{4 s}^{\mathrm{UV}}\left(\delta m_{2} M_{2^{\star}}+B_{2}^{\mathrm{UV}} M_{2}\right)+L_{2}^{\mathrm{UV}} \delta m_{2} \delta m_{2^{\star}}^{\mathrm{UV}} M_{2^{\star}} \\
& +L_{2}^{\mathrm{UV}} B_{2}^{\mathrm{UV}}\left(\delta m_{2^{\prime}}^{\mathrm{UV}} M_{2^{\star}}+B_{2^{\prime}}^{\mathrm{UV}} M_{2}\right)+B_{2}^{\mathrm{UV}} L_{2^{\prime}}^{\mathrm{UV}}\left(\delta m_{2} M_{2^{\star}}+B_{2}^{\mathrm{UV}} M_{2}\right) \\
& +L_{6 c 2}^{\mathrm{R}} M_{2}-L_{2}^{\mathrm{R}} L_{4 c}^{\mathrm{R}} M_{2}+L_{2}^{\mathrm{R}}\left(\Delta M_{6 d}+L_{4 c}^{\mathrm{R}} M_{2}\right)+M_{2^{\star}} \Delta \delta m_{6 d} \\
& M_{29}=\Delta M_{29}+2 \delta m_{2} M_{6 e\left(2^{\star}\right)}+2 B_{2}^{\mathrm{UV}} M_{6 e}+2 L_{6 a 1}^{\mathrm{UV}} M_{2}-\delta m_{2}\left(\delta m_{2} M_{4 a\left(2^{\star \star}\right)}+B_{2}^{\mathrm{UV}} M_{4 a\left(2^{\star}\right)}\right) \\
& -B_{2}^{\mathrm{UV}}\left(\delta m_{2} M_{4 a\left(2^{\star}\right)}+B_{2}^{\mathrm{UV}} M_{4 a}\right)-2 B_{2}^{\mathrm{UV}}\left(L_{4 s\left(3^{\prime}\right)}^{\mathrm{UV}}+L_{4 s\left(1^{\prime \prime}\right)}^{\mathrm{UV}}\right) M_{2}+2\left(B_{2}^{\mathrm{UV}}\right)^{2} L_{2^{\prime \prime}}^{\mathrm{UV}} M_{2} \\
& +2 L_{6 e 2}^{\mathrm{R}} M_{2} \\
& M_{30}=\Delta M_{30}+2 \delta m_{2} M_{6 b\left(2^{\star}\right)}+2 B_{2}^{\mathrm{UV}} M_{6 b}+\delta m_{6 a}^{\mathrm{UV}} M_{2^{\star}}+B_{6 a}^{\mathrm{UV}} M_{2} \\
& -\delta m_{2}\left(\delta m_{2} M_{4 b\left(2^{\star \star}\right)}+B_{2}^{\mathrm{UV}} M_{4 b\left(2^{\star}\right)}\right)-B_{2}^{\mathrm{UV}}\left(\delta m_{2} M_{4 b\left(2^{\star}\right)}+B_{2}^{\mathrm{UV}} M_{4 b}\right) \\
& -2 \delta m_{2} \delta m_{4 b\left(1^{\star}\right)}^{\mathrm{UV}} M_{2^{\star}}-2 B_{2}^{\mathrm{UV}}\left(\delta m_{4 b\left(1^{\prime}\right)}^{\mathrm{UV}} M_{2^{\star}}+B_{4 b\left(1^{\prime}\right)}^{\mathrm{UV}} M_{2}\right) \\
& +2 \delta m_{2} B_{2}^{\mathrm{UV}} \delta m_{2^{\prime \star}}^{\mathrm{UV}} M_{2^{\star}}+\left(B_{2}^{\mathrm{UV}}\right)^{2}\left(\delta m_{2^{\prime \prime}}^{\mathrm{UV}} M_{2^{\star}}+B_{2^{\prime \prime}}^{\mathrm{UV}} M_{2}\right) \\
& +2 L_{6 b 2}^{\mathrm{R}} M_{2}-2 L_{2}^{\mathrm{R}} L_{4 s}^{\mathrm{R}} M_{2}+L_{2}^{\mathrm{R}}\left(\Delta M_{6 a}+2 L_{4 s}^{\mathrm{R}} M_{2}\right)+M_{2^{\star}} \Delta \delta m_{6 a}
\end{aligned}
$$




$$
\begin{aligned}
& M_{31}=\Delta M_{31}+2 L_{6 h 3}^{\mathrm{UV}} M_{2} \\
& M_{32}=\Delta M_{32}+L_{6 g 3}^{\mathrm{UV}} M_{2}+L_{6 h 2}^{\mathrm{UV}} M_{2} \\
& M_{33}=\Delta M_{33}+2 L_{6 g 3}^{\mathrm{UV}} M_{2} \\
& M_{34}=\Delta M_{34}+L_{4 x}^{\mathrm{UV}} M_{4 a}+L_{6 c 3}^{\mathrm{UV}} M_{2}+L_{6 h 1}^{\mathrm{UV}} M_{2}-2 L_{4 x}^{\mathrm{UV}} L_{2}^{\mathrm{UV}} M_{2} \\
& M_{35}=\Delta M_{35}+L_{2}^{\mathrm{UV}} M_{6 h}+L_{6 e 3}^{\mathrm{UV}} M_{2}+L_{6 g 4}^{\mathrm{UV}} M_{2}-2 L_{4 x}^{\mathrm{UV}} L_{2}^{\mathrm{UV}} M_{2} \\
& M_{36}=\Delta M_{36}+L_{2}^{\mathrm{UV}} M_{6 g}+L_{4 l}^{\mathrm{UV}} M_{4 a}+L_{6 b 3}^{\mathrm{UV}} M_{2}+L_{6 g 5}^{\mathrm{UV}} M_{2}-\left(L_{2}^{\mathrm{UV}}\right)^{2} M_{4 a} \\
& -3 L_{2}^{\mathrm{UV}} L_{4 l}^{\mathrm{UV}} M_{2}-L_{2}^{\mathrm{UV}} L_{4 c}^{\mathrm{UV}} M_{2}+2\left(L_{2}^{\mathrm{UV}}\right)^{3} M_{2} \\
& M_{37}=\Delta M_{37}+2 L_{6 g 2}^{\mathrm{UV}} M_{2} \\
& M_{38}=\Delta M_{38}+2 L_{4 x}^{\mathrm{UV}} M_{4 b}+\delta m_{6 h}^{\mathrm{UV}} M_{2^{\star}}+B_{6 h}^{\mathrm{UV}} M_{2}-2 L_{4 x}^{\mathrm{UV}}\left(\delta m_{2} M_{2^{\star}}+B_{2}^{\mathrm{UV}} M_{2}\right) \\
& +L_{2}^{\mathrm{R}} \Delta M_{6 h}+M_{2^{\star}} \Delta \delta m_{6 h} \\
& M_{39}=\Delta M_{39}+L_{2}^{\mathrm{UV}} M_{6 g}+L_{4 c}^{\mathrm{UV}} M_{4 a}+L_{6 g 1}^{\mathrm{UV}} M_{2}+L_{6 c 2}^{\mathrm{UV}} M_{2}-\left(L_{2}^{\mathrm{UV}}\right)^{2} M_{4 a} \\
& -3 L_{2}^{\mathrm{UV}} L_{4 c}^{\mathrm{UV}} M_{2}-L_{2}^{\mathrm{UV}} L_{4 l}^{\mathrm{UV}} M_{2}+2\left(L_{2}^{\mathrm{UV}}\right)^{3} M_{2} \\
& M_{40}=\Delta M_{40}+L_{2}^{\mathrm{UV}} M_{6 c}+L_{4 l}^{\mathrm{UV}} M_{4 b}+L_{4 c}^{\mathrm{UV}} M_{4 b}+\delta m_{6 g}^{\mathrm{UV}} M_{2^{\star}}+B_{6 g}^{\mathrm{UV}} M_{2} \\
& -2\left(L_{2}^{\mathrm{UV}}\right)^{2} M_{4 b}-L_{2}^{\mathrm{UV}}\left(\delta m_{4 a}^{\mathrm{UV}} M_{2^{\star}}+B_{4 a}^{\mathrm{UV}} M_{2}\right)-L_{4 l}^{\mathrm{UV}}\left(\delta m_{2} M_{2^{\star}}+B_{2}^{\mathrm{UV}} M_{2}\right) \\
& -L_{4 c}^{\mathrm{UV}}\left(\delta m_{2} M_{2^{\star}}+B_{2}^{\mathrm{UV}} M_{2}\right)+2\left(L_{2}^{\mathrm{UV}}\right)^{2}\left(\delta m_{2} M_{2^{\star}}+B_{2}^{\mathrm{UV}} M_{2}\right) \\
& +L_{2}^{\mathrm{R}} \Delta M_{6 g}+M_{2^{\star}} \Delta \delta m_{6 g} \\
& M_{41}=\Delta M_{41}+2 L_{2}^{\mathrm{UV}} M_{6 e}+\delta m_{4 a}^{\mathrm{UV}} M_{4 a\left(2^{\star}\right)}+B_{4 a}^{\mathrm{UV}} M_{4 a}+2 L_{6 c 1}^{\mathrm{UV}} M_{2} \\
& -2 L_{2}^{\mathrm{UV}}\left(\delta m_{2} M_{4 a\left(2^{\star}\right)}+B_{2}^{\mathrm{UV}} M_{4 a}\right)-4 L_{2}^{\mathrm{UV}} L_{4 s}^{\mathrm{UV}} M_{2}-2 B_{4 a}^{\mathrm{UV}} L_{2^{\prime}}^{\mathrm{UV}} M_{2} \\
& +4 L_{2}^{\mathrm{UV}} B_{2}^{\mathrm{UV}} L_{2^{\prime}}^{\mathrm{UV}} M_{2} \\
& +L_{4 x}^{\mathrm{R}} \Delta M_{4 a}+\underline{M}_{4 a\left(2^{\star}\right)} \Delta \delta m_{4 a}
\end{aligned}
$$




$$
\begin{aligned}
& M_{42}=\Delta M_{42}+2 L_{2}^{\mathrm{UV}} M_{6 b}+\delta m_{4 a}^{\mathrm{UV}} M_{4 b\left(2^{\star}\right)}+B_{4 a}^{\mathrm{UV}} M_{4 b}+\delta m_{6 c}^{\mathrm{UV}} M_{2^{\star}}+B_{6 c}^{\mathrm{UV}} M_{2} \\
& -2 L_{2}^{\mathrm{UV}}\left(\delta m_{2} M_{4 b\left(2^{\star}\right)}+B_{2}^{\mathrm{UV}} M_{4 b}\right)-2 L_{2}^{\mathrm{UV}}\left(\delta m_{4 b}^{\mathrm{UV}} M_{2^{\star}}+B_{4 b}^{\mathrm{UV}} M_{2}\right) \\
& -\delta m_{4 a}^{\mathrm{UV}} \delta m_{2^{\star}}^{\mathrm{UV}} M_{2^{\star}}-B_{4 a}^{\mathrm{UV}}\left(\delta m_{2^{\prime}}^{\mathrm{UV}} M_{2^{\star}}+B_{2^{\prime}}^{\mathrm{UV}} M_{2}\right) \\
& +2 L_{2}^{\mathrm{UV}} \delta m_{2} \delta m_{2^{\star}}^{\mathrm{UV}} M_{2^{\star}}+2 L_{2}^{\mathrm{UV}} B_{2}^{\mathrm{UV}}\left(\delta m_{2^{\prime}}^{\mathrm{UV}} M_{2^{\star}}+B_{2^{\prime}}^{\mathrm{UV}} M_{2}\right) \\
& +L_{4 l}^{\mathrm{R}} \Delta M_{4 a}-\left(L_{2}^{\mathrm{R}}\right)^{2} \Delta M_{4 a}+L_{2}^{\mathrm{R}}\left(\Delta M_{6 c}+L_{2}^{\mathrm{R}} \Delta M_{4 a}+M_{2^{\star}} \Delta \delta m_{4 a}\right) \\
& +M_{2^{\star}}\left(\Delta \delta m_{6 c}+L_{2}^{\mathrm{R}} \delta m_{4 a}^{\mathrm{R}}\right)+\underline{M}_{4 b\left(2^{\star}\right)} \Delta \delta m_{4 a} \\
& -\delta m_{2^{\star}}^{\mathrm{R}} \Delta \delta m_{4 a} M_{2^{\star}}-\Delta \delta m_{4 a} L_{2}^{\mathrm{R}} M_{2^{\star}} \\
& M_{43}=\Delta M_{43}+\delta m_{2} M_{6 h\left(3^{\star}\right)}+B_{2}^{\mathrm{UV}} M_{6 h}+2 L_{6 e 2}^{\mathrm{UV}} M_{2}-2 B_{2}^{\mathrm{UV}} L_{4 x\left(2^{\prime}\right)}^{\mathrm{UV}} M_{2} \\
& +L_{6 h 3}^{\mathrm{R}} M_{2} \\
& \begin{aligned}
M_{44} & =\Delta M_{44}+\delta m_{2} M_{6 g\left(3^{\star}\right)}+B_{2}^{\mathrm{UV}} M_{6 g}+L_{4 s}^{\mathrm{UV}} M_{4 a}+L_{6 b 2}^{\mathrm{UV}} M_{2}+L_{6 e 1}^{\mathrm{UV}} M_{2} \\
& -B_{2}^{\mathrm{UV}} L_{2^{\prime}}^{\mathrm{UV}} M_{4 a}-B_{2}^{\mathrm{UV}} L_{4 l\left(2^{\prime}\right)}^{\mathrm{UV}} M_{2}-B_{2}^{\mathrm{UV}} L_{4 c\left(2^{\prime}\right)}^{\mathrm{UV}} M_{2}-2 L_{4 s}^{\mathrm{UV}} L_{2}^{\mathrm{UV}} M_{2} \\
& +2 L_{2}^{\mathrm{UV}} B_{2}^{\mathrm{UV}} L_{2^{\prime}}^{\mathrm{UV}} M_{2} \\
& +L_{6 g 3}^{\mathrm{R}} M_{2}
\end{aligned} \\
& M_{45}=\Delta M_{45}+\delta m_{2} M_{6 c\left(3^{\star}\right)}+B_{2}^{\mathrm{UV}} M_{6 c}+2 L_{4 s}^{\mathrm{UV}} M_{4 b}+\delta m_{6 e}^{\mathrm{UV}} M_{2^{\star}}+B_{6 e}^{\mathrm{UV}} M_{2} \\
& -2 B_{2}^{\mathrm{UV}} L_{2^{\prime}}^{\mathrm{UV}} M_{4 b}-\delta m_{2} \delta m_{4 a\left(2^{\star}\right)}^{\mathrm{UV}} M_{2^{\star}}-B_{2}^{\mathrm{UV}}\left(\delta m_{4 a\left(2^{\prime}\right)}^{\mathrm{UV}} M_{2^{\star}}+B_{4 a\left(2^{\prime}\right)}^{\mathrm{UV}} M_{2}\right) \\
& -2 L_{4 s}^{\mathrm{UV}}\left(\delta m_{2} M_{2^{\star}}+B_{2}^{\mathrm{UV}} M_{2}\right)+2 B_{2}^{\mathrm{UV}} L_{2^{\prime}}^{\mathrm{UV}}\left(\delta m_{2} M_{2^{\star}}+B_{2}^{\mathrm{UV}} M_{2}\right) \\
& +L_{6 c 3}^{\mathrm{R}} M_{2}+L_{2}^{\mathrm{R}}\left(\Delta M_{6 e}+L_{4 x}^{\mathrm{R}} M_{2}\right)+M_{2^{\star}} \Delta \delta m_{6 e}-L_{4 x}^{\mathrm{R}} L_{2}^{\mathrm{R}} M_{2} \\
& M_{46}=\Delta M_{46}+\delta m_{2} M_{6 e\left(3^{\star}\right)}+B_{2}^{\mathrm{UV}} M_{6 e}+\delta m_{4 b}^{\mathrm{UV}} M_{4 a\left(2^{\star}\right)}+B_{4 b}^{\mathrm{UV}} M_{4 a}+2 L_{6 b 1}^{\mathrm{UV}} M_{2} \\
& -\delta m_{2} \delta m_{2^{\star}}^{\mathrm{UV}} M_{4 a\left(2^{\star}\right)}-B_{2}^{\mathrm{UV}}\left(\delta m_{2^{\prime}}^{\mathrm{UV}} M_{4 a\left(2^{\star}\right)}+B_{2^{\prime}}^{\mathrm{UV}} M_{4 a}\right)-2 B_{2}^{\mathrm{UV}} L_{4 s\left(2^{\prime}\right)}^{\mathrm{UV}} M_{2} \\
& -2 B_{4 b}^{\mathrm{UV}} L_{2^{\prime}}^{\mathrm{UV}} M_{2}+2 B_{2}^{\mathrm{UV}} B_{2^{\prime}}^{\mathrm{UV}} L_{2^{\prime}}^{\mathrm{UV}} M_{2} \\
& +L_{6 e 3}^{\mathrm{R}} M_{2}-L_{4 x}^{\mathrm{R}} L_{2}^{\mathrm{R}} M_{2}+L_{4 x}^{\mathrm{R}}\left(\Delta M_{4 b}+L_{2}^{\mathrm{R}} M_{2}\right)+\underline{M}_{4 a\left(2^{\star}\right)} \Delta \delta m_{4 b}
\end{aligned}
$$




$$
\begin{aligned}
M_{47} & =\Delta M_{47}+\delta m_{2} M_{6 b\left(3^{\star}\right)}+B_{2}^{\mathrm{UV}} M_{6 b}+\delta m_{4 b}^{\mathrm{UV}} M_{4 b\left(2^{\star}\right)}+B_{4 b}^{\mathrm{UV}} M_{4 b}+\delta m_{6 b}^{\mathrm{UV}} M_{2^{\star}} \\
& +B_{6 b}^{\mathrm{UV}} M_{2}-\delta m_{2} \delta m_{2^{\star}}^{\mathrm{UV}} M_{4 b\left(2^{\star}\right)}-B_{2}^{\mathrm{UV}}\left(\delta m_{2^{\prime}}^{\mathrm{UV}} M_{4 b\left(2^{\star}\right.}+B_{2^{\prime}}^{\mathrm{UV}} M_{4 b}\right) \\
& -\delta m_{2} \delta m_{4 b\left(2^{\star}\right)}^{\mathrm{UV}} M_{2^{\star}}-B_{2}^{\mathrm{UV}}\left(\delta m_{4 b\left(2^{\prime}\right)}^{\mathrm{UV}} M_{2^{\star}}+B_{4 b\left(2^{\prime}\right)}^{\mathrm{UV}} M_{2}\right)-\delta m_{4 b}^{\mathrm{UV}} \delta m_{2^{\star}}^{\mathrm{UV}} M_{2^{\star}} \\
& -B_{4 b}^{\mathrm{UV}}\left(\delta m_{2^{\prime}}^{\mathrm{UV}} M_{2^{\star}}+B_{2^{\prime}}^{\mathrm{UV}} M_{2}\right)+\delta m_{2}\left(\delta m_{2^{\star}}^{\mathrm{UV}}\right)^{2} M_{2^{\star}}+B_{2}^{\mathrm{UV}} \delta m_{2^{\prime}}^{\mathrm{UV}} \delta m_{2^{\star}}^{\mathrm{UV}} M_{2^{\star}} \\
& +B_{2}^{\mathrm{UV}} B_{2^{\prime}}^{\mathrm{UV}}\left(\delta m_{2^{\prime}}^{\mathrm{UV}} M_{2^{\star}}+B_{2^{\prime}}^{\mathrm{UV}} M_{2}\right) \\
& +M_{2^{\star}}\left[\Delta \delta m_{6 b}+L_{2}^{\mathrm{R}}\left\{\delta m_{4 b}^{\mathrm{R}}-\left(\delta m_{2} \delta m_{2^{\star}}^{\mathrm{R}}+B_{2}^{\mathrm{UV}} \delta m_{2^{\prime}}^{\mathrm{R}}\right)\right\}\right] \\
& +L_{2}^{\mathrm{R}}\left(\Delta M_{6 b}+M_{2^{\star}} \Delta \delta m_{4 b}+L_{2}^{\mathrm{R}} \Delta M_{4 b}+L_{4 l}^{\mathrm{R}} M_{2}\right)+M_{4 b\left(2^{\star}\right)}^{\mathrm{R}} \Delta \delta m_{4 b} \\
& +L_{4 l}^{\mathrm{R}}\left(\Delta M_{4 b}+L_{2}^{\mathrm{R}} M_{2}\right)+L_{6 b 3}^{\mathrm{R}} M_{2}-\Delta \delta m_{4 b} \delta m_{2^{\star}}^{\mathrm{R}} M_{2^{\star}}-L_{2}^{\mathrm{R}} \Delta \delta m_{4 b} M_{2^{\star}} \\
& -\left(L_{2}^{\mathrm{R}}\right)^{2}\left(\Delta M_{4 b}+L_{2}^{\mathrm{R}} M_{2}\right)-2 L_{4 l}^{\mathrm{R}} L_{2}^{\mathrm{R}} M_{2}+\left(L_{2}^{\mathrm{R}}\right)^{3} M_{2}
\end{aligned}
$$

\section{APPENDIX C: DIVERGENCE STRUCTURE OF THE RENORMALIZATION}

\section{CONSTANTS}

1. Second-order renormalization constants

$$
\begin{aligned}
& L_{2}=L_{2}^{\mathrm{UV}}+\widetilde{L}_{2}, \quad L_{2}^{\mathrm{R}}=\widetilde{L}_{2}=I_{2} \\
& B_{2}=B_{2}^{\mathrm{UV}}+\widetilde{B}_{2}, \quad B_{2}^{\mathrm{R}}=\widetilde{B}_{2}=-I_{2}+\Delta B_{2} \\
& L_{2}^{\mathrm{R}}+B_{2}^{\mathrm{R}}=\Delta B_{2} \\
& B_{2^{\star}}=-2 L_{2^{\star}}, \quad L_{2^{\star}}=I_{2^{\star}}+\Delta L_{2^{\star}} \\
& B_{2^{\star \star}}=-2\left(2 L_{2^{\star \star \dagger}}+L_{2^{\star \dagger \star}}\right) \\
& \delta m_{2^{\star}}=\delta m_{2^{\star}}^{\mathrm{UV}}+I_{2}+\Delta \delta m_{2^{\star}}
\end{aligned}
$$

\section{Fourth-order renormalization constants}

$$
\begin{aligned}
& L_{4 x}=L_{4 x}^{\mathrm{UV}}+I_{4 x}+\Delta L_{4 x} \\
& L_{4 c}=L_{4 c}^{\mathrm{UV}}+I_{4 c}+\Delta L_{4 c}+L_{2}^{\mathrm{UV}} \widetilde{L}_{2} \\
& B_{4 a}=B_{4 a}^{\mathrm{UV}}-I_{4 x}+\Delta B_{4 a}+2 L_{2}^{\mathrm{UV}} \widetilde{B}_{2}-2 I_{4 c}
\end{aligned}
$$




$$
\begin{aligned}
& L_{4 l}=L_{4 l}^{\mathrm{UV}}+I_{4 l}+\left(L_{2}^{\mathrm{R}}\right)^{2}+\Delta L_{4 l}+\widetilde{L}_{2} L_{2}^{\mathrm{UV}} \\
& L_{4 s}=L_{4 s}^{\mathrm{UV}}+I_{4 s}+\Delta L_{4 s}+\delta m_{2} L_{2^{\star}}+B_{2}^{\mathrm{UV}} \widetilde{L}_{2^{\prime}} \\
& B_{4 b}=B_{4 b}^{\mathrm{UV}}+\Delta B_{4 b}+\delta m_{2} B_{2^{\star}}+B_{2}^{\mathrm{UV}} \widetilde{B}_{2^{\prime}}+L_{2}^{\mathrm{R}} \widetilde{B}_{2}-2 I_{4 s}-I_{4 l} \\
& \Delta L B_{4 a}=2 L_{4 c}^{\mathrm{R}}+L_{4 x}^{\mathrm{R}}+B_{4 a}^{\mathrm{R}}=2 \Delta L_{4 c}+\Delta L_{4 x}+\Delta B_{4 a} \\
& \Delta L B_{4 b}=2 L_{4 s}^{\mathrm{R}}+L_{4 l}^{\mathrm{R}}+B_{4 a}^{\mathrm{R}}-L_{2}^{\mathrm{R}} \Delta B_{2}=2 \Delta L_{4 s}+\Delta L_{4 l}+\Delta B_{4 a} \\
& \Delta L B^{(4)}=\Delta L B_{4 a}+\Delta L B_{4 b}=\Delta L^{(4)}+\Delta B^{(4)}
\end{aligned}
$$

\section{Sixth-order renormalization constants}

$$
\begin{aligned}
L_{6 a 1} & =L_{6 a 1}^{\mathrm{R}}+2 \delta m_{2} L_{4 s\left(1^{\star}\right)}+2 B_{2}^{\mathrm{UV}} \widetilde{L}_{4 s\left(1^{\prime}\right)}-\delta m_{2}\left(\delta m_{2} L_{2^{\star \star \dagger}}+B_{2}^{\mathrm{UV}} L_{2^{\prime \star}}\right) \\
& -B_{2}^{\mathrm{UV}}\left(\delta m_{2} L_{2^{\prime \star}}+B_{2}^{\mathrm{UV}} \widetilde{L}_{2^{\prime \prime}}\right)+L_{6 a 1}^{\mathrm{UV}} \\
L_{6 a 2} & =L_{6 a 2}^{\mathrm{R}}+L_{2}^{\mathrm{UV}} \widetilde{L}_{4 s}+\delta m_{2} L_{4 l\left(1^{\star}\right)}+B_{2}^{\mathrm{UV}} \widetilde{L}_{4 l\left(1^{\prime}\right)}-L_{2}^{\mathrm{UV}}\left(\delta m_{2} L_{2^{\star}}+B_{2}^{\mathrm{UV}} \widetilde{L}_{2^{\prime}}\right)+L_{6 a 2}^{\mathrm{UV}} \\
L_{6 a 3} & =L_{6 a 3}^{\mathrm{R}}+2\left(\delta m_{2} L_{4 s\left(1^{\star}\right)}+B_{2}^{\mathrm{UV}} \widetilde{L}_{4 s\left(1^{\prime}\right)}\right)-\delta m_{2}\left(\delta m_{2} L_{2^{\star \dagger \star}}+B_{2}^{\mathrm{UV}} L_{2^{\prime \star}}\right) \\
& -B_{2}^{\mathrm{UV}}\left(\delta m_{2} L_{2^{\prime \star}}+B_{2}^{\mathrm{UV}} \widetilde{L}_{2^{\prime \prime}}\right)+L_{6 a 3}^{\mathrm{UV}} \\
L_{6 b 1} & =L_{6 b 1}^{\mathrm{R}}+\delta m_{2} L_{4 s\left(2^{\star}\right)}+B_{2}^{\mathrm{UV}} \widetilde{L}_{4 s\left(2^{\prime}\right)}+\delta m_{4 b}^{\mathrm{UV}} L_{2^{\star}}+B_{4 b}^{\mathrm{UV}} \widetilde{L}_{2^{\prime}}-\delta m_{2} \delta m_{2^{\star}}^{\mathrm{UV}} L_{2^{\star}} \\
& -B_{2}^{\mathrm{UV}}\left(\delta m_{2^{\prime}}^{\mathrm{UV}} L_{2^{\star}}+B_{2^{\prime}}^{\mathrm{UV}} \widetilde{L}_{2^{\prime}}\right)+L_{6 b 1}^{\mathrm{UV}} \\
L_{6 b 2} & =L_{6 b 2}^{\mathrm{R}}+\delta m_{2} L_{4 l\left(2^{\star}\right)}+B_{2}^{\mathrm{UV}} \widetilde{L}_{4 l\left(2^{\prime}\right)}+L_{4 s}^{\mathrm{UV}} \widetilde{L}_{2}-B_{2}^{\mathrm{UV}} L_{2^{\prime}}^{\mathrm{UV}} \widetilde{L}_{2}+L_{6 b 2}^{\mathrm{UV}} \\
L_{6 b 3} & =L_{6 b 3}^{\mathrm{R}}+L_{2}^{\mathrm{UV}} \widetilde{L}_{4 l}+L_{4 l}^{\mathrm{UV}} \widetilde{L}_{2}-\left(L_{2}^{\mathrm{UV}}\right)^{2} \widetilde{L}_{2}+L_{6 b 3}^{\mathrm{UV}} \\
L_{6 c 3} & =L_{6 c 3}^{\mathrm{R}}+L_{4 x}^{\mathrm{UV}} \widetilde{L}_{2}+L_{6 c 3}^{\mathrm{UV}} \\
L_{6 c 1} & =L_{6 c 1}^{\mathrm{R}}+2 L_{2}^{\mathrm{UV}} \widetilde{L}_{4 s}+\delta m_{4 a}^{\mathrm{UV}} L_{2^{\star}}+B_{4 a}^{\mathrm{UV}} \widetilde{L}_{2^{\prime}}-2 L_{2}^{\mathrm{UV}}\left(\delta m_{2} L_{2^{\star}}+B_{2}^{\mathrm{UV}} \widetilde{L}_{2^{\prime}}\right)+L_{6 c 1}^{\mathrm{UV}} \\
L_{6 c 2} & =L_{6 c 2}^{\mathrm{R}}+L_{2}^{\mathrm{UV}} \widetilde{L}_{4 l}+L_{4 c}^{\mathrm{UV}} \widetilde{L}_{2}-\left(L_{2}^{\mathrm{UV}}\right)^{2} \widetilde{L}_{2}+L_{6 c 2}^{\mathrm{UV}} \\
& =\delta m_{2} L_{4 c\left(1^{\star}\right)}+B_{2}^{\mathrm{UV}} \widetilde{L}_{4 c\left(1^{\prime}\right)}+L_{2}^{\mathrm{UV}} \widetilde{L}_{4 s}-L_{2}^{\mathrm{UV}}\left(\delta m_{2} L_{2^{\star}}+B_{2}^{\mathrm{UV}} \widetilde{L}_{2^{\prime}}\right)+L_{6 d 1}^{\mathrm{UV}}
\end{aligned}
$$




$$
\begin{aligned}
& L_{6 d 2}=L_{6 d 2}^{\mathrm{R}}+L_{2}^{\mathrm{UV}} \widetilde{L}_{4 c}+L_{2}^{\mathrm{UV}} \widetilde{L}_{4 l}-\left(L_{2}^{\mathrm{UV}}\right)^{2} \widetilde{L}_{2}+L_{6 d 2}^{\mathrm{UV}} \\
& L_{6 d 3}=L_{6 d 3}^{\mathrm{R}}+\delta m_{2} L_{4 c\left(1^{\star}\right)}+B_{2}^{\mathrm{UV}} \widetilde{L}_{4 c\left(1^{\prime}\right)}+L_{2}^{\mathrm{UV}} \widetilde{L}_{4 s}-L_{2}^{\mathrm{UV}}\left(\delta m_{2} L_{2^{\star}}+B_{2}^{\mathrm{UV}} \widetilde{L}_{2^{\prime}}\right)+L_{6 d 3}^{\mathrm{UV}} \\
& L_{6 d 4}=L_{6 d 4}^{\mathrm{R}}+\delta m_{2} L_{4 x\left(1^{\star}\right)}+B_{2}^{\mathrm{UV}} \widetilde{L}_{4 x\left(1^{\prime}\right)}+L_{6 d 4}^{\mathrm{UV}} \\
& L_{6 d 5}=L_{6 d 5}^{\mathrm{R}}+\delta m_{2} L_{4 c\left(3^{\star}\right)}+B_{2}^{\mathrm{UV}} \widetilde{L}_{4 c\left(3^{\prime}\right)}+L_{4 s}^{\mathrm{UV}} \widetilde{L}_{2}-B_{2}^{\mathrm{UV}} L_{2^{\prime}}^{\mathrm{UV}} \widetilde{L}_{2}+L_{6 d 5}^{\mathrm{UV}} \\
& L_{6 e 1}=L_{6 e 1}^{\mathrm{R}}+\delta m_{2} L_{4 c\left(2^{\star}\right)}+B_{2}^{\mathrm{UV}} \widetilde{L}_{4 c\left(2^{\prime}\right)}+L_{4 s}^{\mathrm{UV}} \widetilde{L}_{2}-B_{2}^{\mathrm{UV}} L_{2^{\prime}}^{\mathrm{UV}} \widetilde{L}_{2}+L_{6 e 1}^{\mathrm{UV}} \\
& L_{6 e 2}=L_{6 e 2}^{\mathrm{R}}+\delta m_{2} L_{4 x\left(2^{\star}\right)}+B_{2}^{\mathrm{UV}} \widetilde{L}_{4 x\left(2^{\prime}\right)}+L_{6 e 2}^{\mathrm{UV}} \\
& L_{6 e 3}=L_{6 e 3}^{\mathrm{R}}+L_{2}^{\mathrm{UV}} \widetilde{L}_{4 x}+L_{6 e 3}^{\mathrm{UV}} \\
& L_{6 f 1}=L_{6 f 1}^{\mathrm{R}}+L_{2}^{\mathrm{UV}} \widetilde{L}_{4 c}+L_{4 c}^{\mathrm{UV}} \widetilde{L}_{2}-\left(L_{2}^{\mathrm{UV}}\right)^{2} \widetilde{L}_{2}+L_{6 f 1}^{\mathrm{UV}} \\
& L_{6 f 2}=L_{6 f 2}^{\mathrm{R}}+L_{2}^{\mathrm{UV}} \widetilde{L}_{4 x}+L_{6 f 2}^{\mathrm{UV}} \\
& L_{6 f 3}=L_{6 f 3}^{\mathrm{R}}+2 L_{2}^{\mathrm{UV}} \widetilde{L}_{4 c}-\left(L_{2}^{\mathrm{UV}}\right)^{2} \widetilde{L}_{2}+L_{6 f 3}^{\mathrm{UV}} \\
& L_{6 g 1}=L_{6 g 1}^{\mathrm{R}}+L_{2}^{\mathrm{UV}} \widetilde{L}_{4 c}+L_{4 c}^{\mathrm{UV}} \widetilde{L}_{2}-\left(L_{2}^{\mathrm{UV}}\right)^{2} \widetilde{L}_{2}+L_{6 g 1}^{\mathrm{UV}} \\
& L_{6 g 2}=L_{6 g 2}^{\mathrm{R}}+L_{6 g 2}^{\mathrm{UV}} \\
& L_{6 g 3}=L_{6 g 3}^{\mathrm{R}}+L_{6 g 3}^{\mathrm{UV}} \\
& L_{6 g 4}=L_{6 g 4}^{\mathrm{R}}+L_{2}^{\mathrm{UV}} \widetilde{L}_{4 x}+L_{6 g 4}^{\mathrm{UV}} \\
& L_{6 g 5}=L_{6 g 5}^{\mathrm{R}}+L_{2}^{\mathrm{UV}} \widetilde{L}_{4 c}+L_{4 l}^{\mathrm{UV}} \widetilde{L}_{2}-\left(L_{2}^{\mathrm{UV}}\right)^{2} \widetilde{L}_{2}+L_{6 g 5}^{\mathrm{UV}} \\
& L_{6 h 1}=L_{6 h 1}^{\mathrm{R}}+L_{4 x}^{\mathrm{UV}} \widetilde{L}_{2}+L_{6 h 1}^{\mathrm{UV}} \\
& L_{6 h 2}=L_{6 h 2}^{\mathrm{R}}+L_{6 h 2}^{\mathrm{UV}}
\end{aligned}
$$




$$
\begin{aligned}
L_{6 h 3} & =L_{6 h 3}^{\mathrm{R}}+L_{6 h 3}^{\mathrm{UV}} \\
B_{6 a} & =B_{6 a}^{\mathrm{R}}+2\left(\delta m_{2} B_{4 b\left(1^{\star}\right)}+B_{2}^{\mathrm{UV}} \widetilde{B}_{4 b\left(1^{\prime}\right)}\right)-\delta m_{2}\left(\delta m_{2} B_{2^{\star \star}}+B_{2}^{\mathrm{UV}} B_{2^{\prime \star}}\right) \\
& -B_{2}^{\mathrm{UV}}\left(\delta m_{2} B_{2^{\prime \star}}+B_{2}^{\mathrm{UV}} \widetilde{B}_{2^{\prime \prime}}\right)+B_{6 a}^{\mathrm{UV}} \\
B_{6 b} & =B_{6 b}^{\mathrm{R}}+\delta m_{2} B_{4 b\left(2^{\star}\right)}+B_{2}^{\mathrm{UV}} \widetilde{B}_{4 b\left(2^{\prime}\right)}+\delta m_{4 b}^{\mathrm{UV}} B_{2^{\star}}+B_{4 b}^{\mathrm{UV}} \widetilde{B}_{2^{\prime}}-\delta m_{2} \delta m_{2^{\star}}^{\mathrm{UV}} B_{2^{\star}} \\
& -B_{2}^{\mathrm{UV}}\left(\delta m_{2^{\prime}}^{\mathrm{UV}} B_{2^{\star}}+B_{2^{\prime}}^{\mathrm{UV}} \widetilde{B}_{2^{\prime}}\right)+B_{6 b}^{\mathrm{UV}} \\
B_{6 c} & =B_{6 c}^{\mathrm{R}}+2 L_{2}^{\mathrm{UV}} \widetilde{B}_{4 b}+\delta m_{4 a}^{\mathrm{UV}} B_{2^{\star}}+B_{4 a}^{\mathrm{UV}} \widetilde{B}_{2^{\prime}}-2 L_{2}^{\mathrm{UV}}\left(\delta m_{2} B_{2^{\star}}+B_{2}^{\mathrm{UV}} \widetilde{B}_{2^{\prime}}\right)+B_{6 c}^{\mathrm{UV}} \\
B_{6 d} & =B_{6 d}^{\mathrm{R}}+\delta m_{2} B_{4 a\left(1^{\star}\right)}+B_{2}^{\mathrm{UV}} \widetilde{B}_{4 a\left(1^{\prime}\right)}+L_{2}^{\mathrm{UV}} \widetilde{B}_{4 b}+L_{4 s}^{\mathrm{UV}} \widetilde{B}_{2}-L_{2}^{\mathrm{UV}}\left(\delta m_{2} B_{2^{\star}}+B_{2}^{\mathrm{UV}} \widetilde{B}_{2^{\prime}}\right) \\
& -B_{2}^{\mathrm{UV}} L_{2^{\prime}}^{\mathrm{UV}} \widetilde{B}_{2}+B_{6 d}^{\mathrm{UV}} \\
B_{6 g} & =B_{6 g}^{\mathrm{R}}+L_{2}^{\mathrm{UV}} \widetilde{B}_{4 a}+L_{4 c}^{\mathrm{UV}} \widetilde{B}_{2}+L_{4 l}^{\mathrm{UV}} \widetilde{B}_{2}-2\left(L_{2}^{\mathrm{UV}}\right)^{2} \widetilde{B}_{2}+B_{6 g}^{\mathrm{UV}} \\
B_{6 f} & =B_{6 f}^{\mathrm{R}}+2 L_{2}^{\mathrm{UV}} \widetilde{B}_{4 a}+2 L_{4 c}^{\mathrm{UV}} \widetilde{B}_{2}-3\left(L_{2}^{\mathrm{UV}}\right)^{2} \widetilde{B}_{2}+B_{6 f}^{\mathrm{UV}} \\
B_{6 e} & =\delta m_{2} B_{4 a\left(2^{\star}\right)}+B_{2}^{\mathrm{UV}} \widetilde{B}_{4 a\left(2^{\prime}\right)}+2 L_{4 s}^{\mathrm{UV}} \widetilde{B}_{2}-2 B_{2}^{\mathrm{UV}} L_{2^{\prime}}^{\mathrm{UV}} \widetilde{B}_{2}+B_{6 e}^{\mathrm{UV}}
\end{aligned}
$$

The residual renormalization constants $\Delta L B_{6 x}$ for each diagram appearing in Eq. (77) are defined in the following equations.

$$
\begin{aligned}
\Delta L B_{6 a} & =2 L_{6 a 1}^{\mathrm{R}}+2 L_{6 a 2}^{\mathrm{R}}+L_{6 a 3}^{\mathrm{R}}+B_{6 a}^{\mathrm{R}} \\
& -2 L_{4 s}^{\mathrm{R}} \Delta B_{2} \\
\Delta L B_{6 b} & =2 L_{6 b 1}^{\mathrm{R}}+2 L_{6 b 2}^{\mathrm{R}}+L_{6 b 3}^{\mathrm{R}}+B_{6 b}^{\mathrm{R}} \\
& -L_{2}^{\mathrm{R}}\left(B_{4 b}^{\mathrm{R}}+2 L_{4 s}^{\mathrm{R}}+L_{4 l}^{\mathrm{R}}\right)-L_{4 l}^{\mathrm{R}} \Delta B_{2}+\left(L_{2}^{\mathrm{R}}\right)^{2} \Delta B_{2}
\end{aligned}
$$




$$
\begin{aligned}
\Delta L B_{6 c} & =2 L_{6 c 1}^{\mathrm{R}}+2 L_{6 c 2}^{\mathrm{R}}+L_{6 c 3}^{\mathrm{R}}+B_{6 c}^{\mathrm{R}} \\
& -L_{2}^{\mathrm{R}}\left(B_{4 a}^{\mathrm{R}}+2 L_{4 c}^{\mathrm{R}}+L_{4 x}^{\mathrm{R}}\right) \\
\Delta L B_{6 d} & =L_{6 d 1}^{\mathrm{R}}+L_{6 d 2}^{\mathrm{R}}+L_{6 d 3}^{\mathrm{R}}+L_{6 d 4}^{\mathrm{R}}+L_{6 d 5}^{\mathrm{R}}+B_{6 d}^{\mathrm{R}} \\
& -L_{4 c}^{\mathrm{R}} \Delta B_{2} \\
\Delta L B_{6 e} & =2 L_{6 e 1}^{\mathrm{R}}+2 L_{6 e 2}^{\mathrm{R}}+L_{6 e 3}^{\mathrm{R}}+B_{6 e}^{\mathrm{R}} \\
& -L_{4 x}^{\mathrm{R}} \Delta B_{2} \\
\Delta L B_{6 f} & =2 L_{6 f 1}^{\mathrm{R}}+2 L_{6 f 2}^{\mathrm{R}}+L_{6 f 3}^{\mathrm{R}}+B_{6 f}^{\mathrm{R}} \\
\Delta L B_{6 g} & =L_{6 g 1}^{\mathrm{R}}+L_{6 g 2}^{\mathrm{R}}+L_{6 g 3}^{\mathrm{R}}+L_{6 g 4}^{\mathrm{R}}+L_{6 g 5}^{\mathrm{R}}+B_{6 g}^{\mathrm{R}} \\
\Delta L B_{6 h} & =2 L_{6 h 1}^{\mathrm{R}}+2 L_{6 h 2}^{\mathrm{R}}+L_{6 h 3}^{\mathrm{R}}+B_{6 h}^{\mathrm{R}} \\
\Delta L B^{(6)} & =\sum_{x=a}^{h} \eta_{x} \Delta L B_{6 x}=\Delta L^{(6)}+\Delta B^{(6)}+\Delta L^{(4)} \Delta B_{2}+\Delta \delta m^{(4)} B_{2^{\star}}[I] \\
\Delta & \\
\Delta & \\
\Delta &
\end{aligned}
$$

[1] P. Kusch and H. M. Foley, Phys. Rev. 72, 1256 (1947).

[2] J. S. Schwinger, Phys. Rev. 73, 416 (1948).

[3] A. Rich and J. C. Wesley, Rev, Mod. Phys. 44, 250 (1972).

[4] R. S. Van Dyck, P. B. Schwinberg, and H. G. Dehmelt, Phys. Rev. Lett. 59, 26 (1987).

[5] L. S. Brown, G. Gabrielse, K. Helmerson, and J. Tan, Phys. Rev. Lett. 55, 44 (1985).

[6] B. Odom, D. Hanneke, B. D’Urso, and G. Gabrielse, Phys. Rev. Lett. 97, 030801 (2006).

[7] D. Hanneke, S. Forgwell, and G. Gabrielse, arXiv:0801.1134[atom-ph] (2008).

[8] M. Davier and A. Höcker, Phys. Lett. B435, 427 (1998), hep-ph/9805470.

[9] B. Krause, Phys. Lett. B390, 392 (1997), hep-ph/9607259.

[10] K. Melnikov and A. Vainshtein, Phys. Rev. D70, 113006 (2004), hep-ph/0312226.

[11] J. Bijnens and J. Prades, Mod. Phys. Lett. A 22, 767 (2007), hep-ph/0702170.

[12] A. Czarnecki, B. Krause, and W. J. Marciano, Phys. Rev. Lett. 76, 3267 (1996), hep$\mathrm{ph} / 9512369$. 
[13] T. Aoyama, M. Hayakawa, T. Kinoshita, and M. Nio, Phys. Rev. Lett. 99, 110406 (2007), arXiv:0706.3496[hep-ph].

[14] T. Aoyama, M. Hayakawa, T. Kinoshita, and M. Nio, Nucl. Phys. B 740, 138 (2006), hep$\mathrm{ph} / 0512288$.

[15] T. Aoyama, M. Hayakawa, T. Kinoshita, and M. Nio (2007), arXiv:0709.1568[hep-ph].

[16] T. Kinoshita and M. Nio, Phys. Rev. D 73, 013003 (2006), hep-ph/0507249.

[17] G. P. Lepage, J. Comput. Phys. 27, 192 (1978).

[18] A. Petermann, Helv. Phys. Acta 30, 407 (1957).

[19] C. M. Sommerfield, Phys. Rev. 107, 328 (1957).

[20] T. Kinoshita, Phys. Rev. Lett. 75, 4728 (1995).

[21] S. Laporta and E. Remiddi, Phys. Lett. B379, 283 (1996), hep-ph/9602417.

[22] M. A. Samuel and G.-W. Li, Phys. Rev. D 44, 3935 (1991).

[23] G. Li, R. Mendel, and M. A. Samuel, Phys. Rev. D 47, 1723 (1993).

[24] A. Czarnecki and M. Skrzypek, Phys. Lett. B449, 354 (1999), hep-ph/9812394.

[25] S. Laporta, Nuovo Cim. A106, 675 (1993).

[26] S. Laporta and E. Remiddi, Phys. Lett. B301, 440 (1993).

[27] B. Lautrup, Phys. Lett. B69, 109 (1977).

[28] M. Passera, Phys. Rev. D 75, 013002 (2007), hep-ph/0606174.

[29] P. J. Mohr and B. N. Taylor, Rev. Mod. Phys. 77, 1 (2005).

[30] A. Wicht et al., Phys. Scr. T 102, 82 (2002).

[31] V. Gerginov et al., Phys. Rev. A. 73, 032504 (2006).

[32] P. Cladé et al., Phys. Rev. A. 74, 052109 (2006).

[33] G. Gabrielse, D. Hanneke, T. Kinoshita, M. Nio, and B. Odom, Phys. Rev. Lett. 97, 030802 (2006).

[34] G. Gabrielse, D. Hanneke, T. Kinoshita, M. Nio, and B. Odom, Phys. Rev. Lett. 99, 039902 (2007).

[35] P. Cvitanović and T. Kinoshita, Phys. Rev. D 10, 3978 (1974).

[36] T. Kinoshita, in Quantum electrodynamics, edited by T. Kinoshita (World Scientific, Singapore, 1990), pp. 218-321, (Advanced series on directions in high energy physics, 7).

[37] P. Cvitanović and T. Kinoshita, Phys. Rev. D 10, 3991 (1974).

[38] T. Kinoshita and W. B. Lindquist, Phys. Rev. D 42, 636 (1990). 
[39] T. Aoyama, M. Hayakawa, T. Kinoshita, and M. Nio (2007), in preparation.

[40] P. Cvitanović and T. Kinoshita, Phys. Rev. D 10, 4007 (1974). 